\title{
Triple Hierarchical Variational Inequalities with Constraints of Mixed Equilibria, Variational Inequalities, Convex Minimization, and Hierarchical Fixed Point Problems
}

\author{
Lu-Chuan Ceng, ${ }^{1}$ Cheng-Wen Liao, ${ }^{2}$ Chin-Tzong Pang, ${ }^{3}$ and Ching-Feng Wen ${ }^{4}$ \\ ${ }^{1}$ Department of Mathematics, Shanghai Normal University and Scientific Computing Key Laboratory of Shanghai Universities, \\ Shanghai 200234, China \\ ${ }^{2}$ Department of Food and Beverage Management, Vanung University, Chung-Li 320061, Taiwan \\ ${ }^{3}$ Department of Information Management, and Innovation Center for Big Data and Digital Convergence, Yuan Ze University, \\ Chung-Li 32003, Taiwan \\ ${ }^{4}$ Center for Fundamental Science, Kaohsiung Medical University, Kaohsiung 807, Taiwan
}

Correspondence should be addressed to Chin-Tzong Pang; imctpang@saturn.yzu.edu.tw

Received 7 April 2014; Accepted 27 April 2014; Published 26 May 2014

Academic Editor: Jen-Chih Yao

Copyright (C) $2014 \mathrm{Lu}$-Chuan Ceng et al. This is an open access article distributed under the Creative Commons Attribution License, which permits unrestricted use, distribution, and reproduction in any medium, provided the original work is properly cited.

\begin{abstract}
We introduce and analyze a hybrid iterative algorithm by virtue of Korpelevich's extragradient method, viscosity approximation method, hybrid steepest-descent method, and averaged mapping approach to the gradient-projection algorithm. It is proven that under appropriate assumptions, the proposed algorithm converges strongly to a common element of the fixed point set of infinitely many nonexpansive mappings, the solution set of finitely many generalized mixed equilibrium problems (GMEPs), the solution set of finitely many variational inequality problems (VIPs), the solution set of general system of variational inequalities (GSVI), and the set of minimizers of convex minimization problem (CMP), which is just a unique solution of a triple hierarchical variational inequality (THVI) in a real Hilbert space. In addition, we also consider the application of the proposed algorithm to solve a hierarchical fixed point problem with constraints of finitely many GMEPs, finitely many VIPs, GSVI, and CMP. The results obtained in this paper improve and extend the corresponding results announced by many others.
\end{abstract}

\section{Introduction}

Let $C$ be a nonempty closed convex subset of a real Hilbert space $H$ and let $P_{C}$ be the metric projection of $H$ onto $C$. Let $S: C \rightarrow H$ be a nonlinear mapping on $C$. We denote by $\operatorname{Fix}(S)$ the set of fixed points of $S$ and by $\mathbf{R}$ the set of all real numbers. A mapping $S: C \rightarrow H$ is called $L$-Lipschitz continuous if there exists a constant $L \geq 0$ such that

$$
\|S x-S y\| \leq L\|x-y\|, \quad \forall x, y \in C .
$$

In particular, if $L=1$, then $S$ is called a nonexpansive mapping; if $L \in[0,1)$, then $S$ is called a contraction.
Let $A: C \rightarrow H$ be a nonlinear mapping on $C$. We consider the following variational inequality problem (VIP): find a point $x \in C$ such that

$$
\langle A x, y-x\rangle \geq 0, \quad \forall y \in C
$$

The solution set of VIP (2) is denoted by $\operatorname{VI}(C, A)$.

The VIP (2) was first discussed by Lions [1]. There are many applications of VIP (2) in various fields; see, for example, [2-5]. It is well known that if $A$ is a strongly monotone and Lipschitz-continuous mapping on $C$, then VIP (2) has a unique solution. In 1976, Korpelevich [6] proposed 
an iterative algorithm for solving the VIP (2) in Euclidean space $\mathbf{R}^{n}$ :

$$
\begin{gathered}
y_{n}=P_{C}\left(x_{n}-\tau A x_{n}\right), \\
x_{n+1}=P_{C}\left(x_{n}-\tau A y_{n}\right), \quad \forall n \geq 0,
\end{gathered}
$$

with $\tau>0$ a given number, which is known as the extragradient method. The literature on the VIP is vast, among which, Korpelevich's extragradient method has received great attention in various applications and undergone improvements in many ways; see, for example, [7-20] and references therein, to name but a few.

Let $\varphi: C \rightarrow \mathbf{R}$ be a real-valued function, let $A$ : $H \rightarrow H$ be a nonlinear mapping, and let $\Theta: C \times C \rightarrow \mathbf{R}$ be a bifunction. In 2008, Peng and Yao [8] introduced the following generalized mixed equilibrium problem (GMEP) of finding $x \in C$ such that

$$
\begin{array}{r}
\Theta(x, y)+\varphi(y)-\varphi(x)+\langle A x, y-x\rangle \geq 0, \\
\forall y \in C .
\end{array}
$$

We denote the set of solutions of GMEP (4) by $\operatorname{GMEP}(\Theta, \varphi, A)$. The GMEP (4) is very general in the sense that it includes, as special cases, optimization problems, variational inequalities, minimax problems, Nash equilibrium problems in noncooperative games, and others. The GMEP is further considered and studied; see, for example, $[10,16,18,19,21-23]$. In particular, if $\varphi=0$, then GMEP (4) is reduced to the generalized equilibrium problem (GEP) which is to find $x \in C$ such that

$$
\Theta(x, y)+\langle A x, y-x\rangle \geq 0, \quad \forall y \in C .
$$

It was introduced and studied by S. Takahashi and W. Takahashi [24]. The set of solutions of GEP is denoted by $\operatorname{GEP}(\Theta, A)$.

If $A=0$, then GMEP (4) is reduced to the mixed equilibrium problem (MEP), which is to find $x \in C$ such that

$$
\Theta(x, y)+\varphi(y)-\varphi(x) \geq 0, \quad \forall y \in C .
$$

It was considered and studied in [25]. The set of solutions of MEP is denoted by $\operatorname{MEP}(\Theta, \varphi)$.

If $\varphi=0$ and $A=0$, then GMEP (4) is reduced to the equilibrium problem (EP), which is to find $x \in C$ such that

$$
\Theta(x, y) \geq 0, \quad \forall y \in C .
$$

It was considered and studied in $[26,27]$. The set of solutions of EP is denoted by $\mathrm{EP}(\Theta)$. It is worth mentioning that the EP is a unified model of several problems, namely, the variational inequality problems, the optimization problems, the saddle point problems, the complementarity problems, the fixed point problems, the Nash equilibrium problems, and so forth.

It was assumed in [8] that $\Theta: C \times C \rightarrow \mathbf{R}$ is a bifunction satisfying conditions (A1)-(A4) and $\varphi: C \rightarrow \mathbf{R}$ is a lower semicontinuous and convex function with a restriction (B1) or (B2), where

$$
\text { (A1) } \Theta(x, x)=0 \text { for all } x \in C \text {; }
$$

(A2) $\Theta$ is monotone; that is, $\Theta(x, y)+\Theta(y, x) \leq 0$ for any $x, y \in C$;

(A3) $\Theta$ is upper-hemicontinuous; that is, for each $x, y, z \in$ C,

$$
\limsup _{t \rightarrow 0^{+}} \Theta(t z+(1-t) x, y) \leq \Theta(x, y) ;
$$

(A4) $\Theta(x, \cdot)$ is convex and lower semicontinuous for each $x \in C$;

(B1) for each $x \in H$ and $r>0$, there exists a bounded subset $D_{x} \subset C$ and $y_{x} \in C$ such that for any $z \in C \backslash D_{x}$,

$$
\Theta\left(z, y_{x}\right)+\varphi\left(y_{x}\right)-\varphi(z)+\frac{1}{r}\left\langle y_{x}-z, z-x\right\rangle<0
$$

(B2) $C$ is a bounded set.

Given a positive number $r>0$. Let $T_{r}^{(\Theta, \varphi)}: H \rightarrow C$ be the solution set of the auxiliary mixed equilibrium problem; that is, for each $x \in H$,

$$
\begin{array}{r}
T_{r}^{(\Theta, \varphi)}(x):=\{y \in C: \Theta(y, z)+\varphi(z)-\varphi(y) \\
\left.+\frac{1}{r}\langle y-x, z-y\rangle \geq 0, \forall z \in C\right\} .
\end{array}
$$

Let $F_{1}, F_{2}: C \rightarrow H$ be two mappings. Consider the following general system of variational inequalities (GSVI) of finding $\left(x^{*}, y^{*}\right) \in C \times C$ such that

$$
\begin{array}{ll}
\left\langle\nu_{1} F_{1} y^{*}+x^{*}-y^{*}, x-x^{*}\right\rangle \geq 0, & \forall x \in C, \\
\left\langle v_{2} F_{2} x^{*}+y^{*}-x^{*}, x-y^{*}\right\rangle \geq 0, & \forall x \in C,
\end{array}
$$

where $v_{1}>0$ and $\nu_{2}>0$ are two constants, which was considered and studied in $[9,11,28]$. In particular, if $F_{1}=F_{2}=$ $A$, then the GSVI (11) is reduced to the following problem of finding $\left(x^{*}, y^{*}\right) \in C \times C$ such that

$$
\begin{array}{ll}
\left\langle\nu_{1} A y^{*}+x^{*}-y^{*}, x-x^{*}\right\rangle \geq 0, & \forall x \in C, \\
\left\langle\nu_{2} A x^{*}+y^{*}-x^{*}, x-y^{*}\right\rangle \geq 0, & \forall x \in C,
\end{array}
$$

which is defined by Verma [29] and called as a new system of variational inequalities (NSVI). Furthermore, if $x^{*}=y^{*}$, then the NSVI reduces to the classical VIP (2). In 2008, Ceng et al. [9] transformed the GSVI (11) into a fixed point problem as follows.

Proposition CWY (see [9]). For given $\bar{x}, \bar{y} \in C,(\bar{x}, \bar{y})$ is a solution of the GSVI (11) if and only if $\bar{x}$ is a fixed point of the mapping $G: C \rightarrow C$ defined by

$$
G x=P_{C}\left(I-v_{1} F_{1}\right) P_{C}\left(I-v_{2} F_{2}\right) x, \quad \forall x \in C,
$$

where $\bar{y}=P_{C}\left(I-v_{2} F_{2}\right) \bar{x}$.

In particular, if the mapping $F_{j}: C \rightarrow H$ is $\zeta_{j}$-inversestrongly monotone for $j=1,2$, then the mapping $G$ is nonexpansive for all $\nu_{j} \in\left(0,2 \zeta_{j}\right], j=1,2$. We denote by $\operatorname{GSVI}(G)$ the fixed point set of the mapping $G$. 
Let $f: C \rightarrow \mathbf{R}$ be a convex and continuously Fréchet differentiable functional. Consider the convex minimization problem (CMP) of minimizing $f$ over the constraint set $C$

$$
\operatorname{minimize}\{f(x): x \in C\},
$$

as considered and studied in [13, 14, 30-32]. We denote by $\Gamma$ the set of minimizers of CMP (14). The gradient-projection algorithm (GPA) generates a sequence $\left\{x_{n}\right\}$ determined by the gradient $\nabla f$ and the metric projection $P_{C}$ :

$$
x_{n+1}:=P_{C}\left(x_{n}-\lambda \nabla f\left(x_{n}\right)\right), \quad \forall n \geq 0
$$

or more generally

$$
x_{n+1}:=P_{C}\left(x_{n}-\lambda_{n} \nabla f\left(x_{n}\right)\right), \quad \forall n \geq 0,
$$

where, in both (15) and (16), the initial guess $x_{0}$ is taken from $C$ arbitrarily and the parameters $\lambda$ or $\lambda_{n}$ are positive real numbers. The convergence of algorithms (15) and (16) depends on the behavior of the gradient $\nabla f$. As a matter of fact, it is known that if $\nabla f$ is $\alpha$-strongly monotone and $L$ Lipschitz continuous, then, for $0<\lambda<2 \alpha / L^{2}$, the operator $P_{C}(I-\lambda \nabla f)$ is a contraction; hence, the sequence $\left\{x_{n}\right\}$ defined by the GPA (15) converges in a norm to the unique solution of CMP (14). More generally, if $\left\{\lambda_{n}\right\}$ is chosen to satisfy the property

$$
0<\liminf _{n \rightarrow \infty} \lambda_{n} \leq \limsup _{n \rightarrow \infty} \lambda_{n}<\frac{2 \alpha}{L^{2}},
$$

then the sequence $\left\{x_{n}\right\}$ defined by the GPA (16) converges in a norm to the unique minimizer of CMP (14). If the gradient $\nabla f$ is only assumed to be Lipschitz continuous, then $\left\{x_{n}\right\}$ can only be weakly convergent if $H$ is infinite-dimensional (a counterexample is given in Section 5 of Xu [31]). Recently, $\mathrm{Xu}$ [31] used averaged mappings to study the convergence analysis of the GPA, which is hence an operator-oriented approach.

Very recently, Ceng and Al-Homidan [23] introduced and analyzed the following iterative algorithm by hybrid steepestdescent viscosity method and derived its strong convergence under appropriate conditions.

Theorem CA (see [23, Theorem 21]). Let $C$ be a nonempty closed convex subset of a real Hilbert space $H$. Let $f: C \rightarrow$ $\mathbf{R}$ be a convex functional with L-Lipschitz continuous gradient $\nabla f$. Let $M, N$ be two integers. Let $\Theta_{k}$ be a bifunction from $C \times C$ to $\mathbf{R}$ satisfying (A1)-(A4) and let $\varphi_{k}: C \rightarrow \mathbf{R} \cup\{+\infty\}$ be a proper lower semicontinuous and convex function, where $k \epsilon$ $\{1,2, \ldots, M\}$. Let $B_{k}: H \rightarrow H$ and $A_{i}: C \rightarrow H$ be $\mu_{k}-$ inverse-strongly monotone and $\eta_{i}$-inverse-strongly monotone, respectively, where $k \in\{1,2, \ldots, M\}$ and $i \in\{1,2, \ldots, N\}$. Let $F: H \rightarrow H$ be a $\kappa$-Lipschitzian and $\eta$-strongly monotone operator with positive constants $\kappa, \eta>0$. Let $V: H \rightarrow H$ be an l-Lipschitzian mapping with a constant $l \geq 0$. Let $0<\mu<$ $2 \eta / \kappa^{2}$ and $0 \leq \gamma l<\tau$, where $\tau=1-\sqrt{1-\mu\left(2 \eta-\mu \kappa^{2}\right)}$. Assume that $\Omega:=\cap_{k=1}^{M} \operatorname{GMEP}\left(\Theta_{k}, \varphi_{k}, B_{k}\right) \cap \cap_{i=1}^{N} V I\left(C, A_{i}\right) \cap \Gamma \neq \emptyset$ and that either (B1) or (B2) holds. For arbitrarily given $x_{1} \in H$, let $\left\{x_{n}\right\}$ be a sequence generated by

$$
\begin{aligned}
& u_{n}= T_{r_{M, n}}^{\left(\Theta_{M}, \varphi_{M}\right)}\left(I-r_{M, n} B_{M}\right) T_{r_{M-1, n}}^{\left(\Theta_{M-1}, \varphi_{M-1}\right)} \\
& \times\left(I-r_{M-1, n} B_{M-1}\right) \cdots T_{r_{1, n}}^{\left(\Theta_{1}, \varphi_{1}\right)}\left(I-r_{1, n} B_{1}\right) x_{n}, \\
& v_{n}= P_{C}\left(I-\lambda_{N, n} A_{N}\right) P_{C}\left(I-\lambda_{N-1, n} A_{N-1}\right) \cdots \\
& \times P_{C}\left(I-\lambda_{2, n} A_{2}\right) P_{C}\left(I-\lambda_{1, n} A_{1}\right) u_{n}, \\
& x_{n+1}= s_{n} \gamma V x_{n}+\beta_{n} x_{n}+\left(\left(1-\beta_{n}\right) I-s_{n} \mu F\right) T_{n} v_{n}, \\
& \forall n \geq 1,
\end{aligned}
$$

where $P_{C}\left(I-\lambda_{n} \nabla f\right)=s_{n} I+\left(1-s_{n}\right) T_{n}$ (here $T_{n}$ is nonexpansive and $s_{n}=\left(2-\lambda_{n} L\right) / 4 \in(0,1 / 2)$ for each $\left.\lambda_{n} \in(0,2 / L)\right)$. Assume that the following conditions hold:

(i) $s_{n} \in(0,1 / 2)$ for each $\lambda_{n} \in(0,2 / L)$, and $\lim _{n \rightarrow \infty} s_{n}=0$ $\left(\Leftrightarrow \lim _{n \rightarrow \infty} \lambda_{n}=2 / L\right)$;

(ii) $\left\{\beta_{n}\right\} \subset(0,1)$ and $0<\liminf _{n \rightarrow \infty} \beta_{n} \leq$ $\limsup _{n \rightarrow \infty} \beta_{n}<1$;

(iii) $\left\{\lambda_{i, n}\right\} \subset\left[a_{i}, b_{i}\right] \subset\left(0,2 \eta_{i}\right)$ and $\lim _{n \rightarrow \infty}\left|\lambda_{i, n+1}-\lambda_{i, n}\right|=0$ for all $i \in\{1,2, \ldots, N\}$;

(iv) $\left\{r_{k, n}\right\} \subset\left[e_{k}, f_{k}\right] \subset\left(0,2 \mu_{k}\right)$ and $\lim _{n \rightarrow \infty}\left|r_{k, n+1}-r_{k, n}\right|=$ 0 for all $k \in\{1,2, \ldots, M\}$.

Then $\left\{x_{n}\right\}$ converges strongly as $\lambda_{n} \rightarrow(2 / L)\left(\Leftrightarrow s_{n} \rightarrow 0\right)$ to a point $x^{*} \in \Omega$, which is a unique solution in $\Omega$ to the VIP:

$$
\left\langle(\mu F-\gamma V) x^{*}, p-x^{*}\right\rangle \geq 0, \quad \forall p \in \Omega .
$$

Equivalently, $x^{*}=P_{\Omega}(I-(\mu F-\gamma V)) x^{*}$.

In 2009, Yao et al. [33] considered the following hierarchical fixed point problem (HFPP): find hierarchically a fixed point of a nonexpansive mapping $T$ with respect to another nonexpansive mapping $S$; namely, find $\widetilde{x} \in \operatorname{Fix}(T)$ such that

$$
\langle\widetilde{x}-S \tilde{x}, \tilde{x}-x\rangle \leq 0, \quad \forall x \in \operatorname{Fix}(T) .
$$

The solution set of HFPP (20) is denoted by $\Lambda$. It is obvious to see that solving HFPP (20) is equivalent to the fixed point problem of the composite mapping $P_{\mathrm{Fix}(T)} S$; that is, find $\tilde{x} \in$ $C$ such that $\tilde{x}=P_{\operatorname{Fix}(T)} S \tilde{x}$. The authors [33] introduced and analyzed the following iterative algorithm for solving HFPP (20):

$$
\begin{gathered}
y_{n}=\beta_{n} S x_{n}+\left(1-\beta_{n}\right) x_{n}, \\
x_{n+1}=\alpha_{n} V x_{n}+\left(1-\alpha_{n}\right) T y_{n}, \quad \forall n \geq 0 .
\end{gathered}
$$

Theorem YLM (see [33, Theorem 3.2]). Let C be a nonempty closed convex subset of a real Hilbert space $H$. Let $S$ and $T$ be two nonexpansive mappings of $C$ into itself. Let $V: C \rightarrow C$ be a fixed contraction with $\alpha \in(0,1)$. Let $\left\{\alpha_{n}\right\}$ and $\left\{\beta_{n}\right\}$ be two sequences in $(0,1)$. For any given $x_{0} \in C$, let $\left\{x_{n}\right\}$ be the sequence generated by (21). Assume that the sequence $\left\{x_{n}\right\}$ is bounded and that

(i) $\sum_{n=0}^{\infty} \alpha_{n}=\infty$; 
(ii) $\lim _{n \rightarrow \infty}\left(1 / \alpha_{n}\right)\left|\left(1 / \beta_{n}\right)-\left(1 / \beta_{n-1}\right)\right|=0$, $\lim _{n \rightarrow \infty}\left(1 / \beta_{n}\right)\left|1-\left(\alpha_{n-1} / \alpha_{n}\right)\right|=0 ;$

(iii) $\lim _{n \rightarrow \infty} \beta_{n}=0, \lim _{n \rightarrow \infty}\left(\alpha_{n} / \beta_{n}\right)=0$ and $\lim _{n \rightarrow \infty}\left(\beta_{n}^{2} / \alpha_{n}\right)=0 ;$

(iv) $\operatorname{Fix}(T) \cap \operatorname{int} C \neq \emptyset$;

(v) there exists a constant $k>0$ such that $\| x-$ $T x \| \geq k \operatorname{Dist}(x, \operatorname{Fix}(T))$ for each $x \in C$, where $\operatorname{Dist}(x, \operatorname{Fix}(T))=\inf _{y \in \operatorname{Fix}(T)}\|x-y\|$. Then $\left\{x_{n}\right\}$ converges strongly to $\tilde{x}=P_{\Lambda} V \tilde{x}$ which solves the VIP $\langle\tilde{x}-S \tilde{x}, \tilde{x}-x\rangle \leq 0$, for all $x \in \operatorname{Fix}(T)$.

Very recently, Iiduka $[34,35]$ considered a variational inequality with a variational inequality constraint over the set of fixed points of a nonexpansive mapping. Since this problem has a triple structure in contrast with the bilevel programming problems or the hierarchical constrained optimization problems or the hierarchical fixed point problem, it is referred to as the triple hierarchical constrained optimization problem (THCOP). He presented some examples of THCOP and developed iterative algorithms to find the solution of such problem. The convergence analysis of the proposed algorithms is also studied in $[34,35]$. Since the original problem is of a variational inequality, in this paper, we call it triple hierarchical variational inequality (THVI). Subsequently, Ceng et al. [36] introduced and considered the following triple hierarchical variational inequality (THVI).

Problem 1. Let $S, T: C \rightarrow C$ be two nonexpansive mappings with $\operatorname{Fix}(T) \neq \emptyset$, let $V: C \rightarrow H$ be a $\rho$-contractive mapping with a constant $\rho \in[0,1)$, and let $F: C \rightarrow H$ be a $\kappa$-Lipschitzian and $\eta$-strongly monotone mapping with constants $\kappa, \eta>0$. Let $0<\mu<2 \eta / \kappa^{2}$ and $0<\gamma \leq \tau$, where $\tau=1-\sqrt{1-\mu\left(2 \eta-\mu \kappa^{2}\right)}$. Consider the following THVI: find $x^{*} \in \Xi$ such that

$$
\left\langle(\mu F-\gamma V) x^{*}, x-x^{*}\right\rangle \geq 0, \quad \forall x \in \Xi,
$$

which $\Xi$ denotes the solution set of the following hierarchical variational inequality (HVI): find $z^{*} \in \operatorname{Fix}(T)$ such that

$$
\left\langle(\mu F-\gamma S) z^{*}, z-z^{*}\right\rangle \geq 0, \quad \forall z \in \operatorname{Fix}(T),
$$

where the solution set $\Xi$ is assumed to be nonempty.

The authors [36] proposed both implicit and explicit iterative methods and studied the convergence analysis of the sequences generated by the proposed methods. In this paper, we introduce and study the following triple hierarchical variational inequality (THVI) with constraints of mixed equilibria, variational inequalities, and convex minimization problem.

Problem 2. Let $M, N$ be two integers. Let $f: C \rightarrow \mathbf{R}$ be a convex functional with $L$-Lipschitz continuous gradient $\nabla f$. Let $\Theta_{k}$ be a bifunction from $C \times C$ to $\mathbf{R}$ and let $\varphi_{k}: C \rightarrow$ $\mathbf{R} \cup\{+\infty\}$ be a proper lower semicontinuous and convex function, where $k \in\{1,2, \ldots, M\}$. Let $B_{k}, A_{i}: H \rightarrow H$ and $F_{j}: C \rightarrow H$ be inverse-strongly monotone mappings, where $k \in\{1,2, \ldots, M\}, i \in\{1,2, \ldots, N\}$, and $j \in\{1,2\}$. Let $S: H \rightarrow H$ be a nonexpansive mapping and let $\left\{S_{n}\right\}_{n=1}^{\infty}$ be a sequence of nonexpansive mappings on $H$. Let $F: H \rightarrow H$ be a $\kappa$-Lipschitzian and $\eta$-strongly monotone operator with positive constants $\kappa, \eta>0$. Let $V: H \rightarrow H$ be an $l$-Lipschitzian mapping with a constant $l \geq 0$. Let $0<\mu<2 \eta / \kappa^{2}, 0<\gamma \leq \tau$, and $0 \leq \gamma l<\tau$, where $\tau=1-\sqrt{1-\mu\left(2 \eta-\mu \kappa^{2}\right)}$. Consider the following triple hierarchical variational inequality (THVI): find $x^{*} \in \Xi$ such that

$$
\left\langle(\mu F-\gamma V) x^{*}, x-x^{*}\right\rangle \geq 0, \quad \forall x \in \Xi,
$$

where $\Xi$ denotes the solution set of the following hierarchical variational inequality (HVI): find $z^{*} \in \Omega:=\left(\cap_{n=1}^{\infty} \operatorname{Fix}\left(S_{n}\right)\right) \cap$ $\left(\cap_{k=1}^{M} \operatorname{GMEP}\left(\Theta_{k}, \varphi_{k}, B_{k}\right)\right) \cap\left(\cap_{i=1}^{N} \operatorname{VI}\left(C, A_{i}\right)\right) \cap \operatorname{GSVI}(G) \cap \Gamma$ such that

$$
\left\langle(\mu F-\gamma S) z^{*}, z-z^{*}\right\rangle \geq 0, \quad \forall z \in \Omega,
$$

where the solution set $\Xi$ is assumed to be nonempty.

Motivated and inspired by the above facts, we introduce and analyze a hybrid iterative algorithm by the virtue of Korpelevich's extragradient method, the viscosity approximation method, the hybrid steepest-descent method, and the averaged mapping approach to the GPA. It is proven that under appropriate assumptions, the proposed algorithm converges strongly to a common element $x^{*} \in \Omega:=\left(\cap_{n=1}^{\infty} \operatorname{Fix}\left(S_{n}\right)\right) \cap$ $\left(\cap_{k=1}^{M} \operatorname{GMEP}\left(\Theta_{k}, \varphi_{k}, B_{k}\right)\right) \cap\left(\cap_{i=1}^{N} \operatorname{VI}\left(C, A_{i}\right)\right) \cap \operatorname{GSVI}(G) \cap \Gamma$ of the fixed point set of infinitely many nonexpansive mappings $\left\{S_{n}\right\}_{n=1}^{\infty}$, the solution set of finitely many GMEPs, the solution set of finitely many VIPs, the solution set of GSVI (11), and the set of minimizers of CMP (14), which is just a unique solution of the THVI (24). In addition, we also consider the application of the proposed algorithm to solve a hierarchical fixed point problem with constraints of finitely many GMEPs, finitely many VIPs, GSVI (11), and CMP (14). That is, under very mild conditions, it is proven that the proposed algorithm converges strongly to a unique solution $x^{*} \in \Omega$ of the VIP: $\left\langle(\gamma V-\mu F) x^{*}, x-x^{*}\right\rangle \leq 0$, for all $x \in \Omega$; equivalently, $P_{\Omega}(I-(\mu F-\gamma V)) x^{*}=x^{*}$. The results obtained in this paper improve and extend the corresponding results announced by many others.

\section{Preliminaries}

Throughout this paper, we assume that $H$ is a real Hilbert space whose inner product and norm are denoted by $\langle\cdot, \cdot\rangle$ and $\|\cdot\|$, respectively. Let $C$ be a nonempty closed convex subset of $H$. We write $x_{n} \rightarrow x$ to indicate that the sequence $\left\{x_{n}\right\}$ converges weakly to $x$ and $x_{n} \rightarrow x$ to indicate that the sequence $\left\{x_{n}\right\}$ converges strongly to $x$. Moreover, we use $\omega_{w}\left(x_{n}\right)$ to denote the weak $\omega$-limit set of the sequence $\left\{x_{n}\right\}$; that is,

$$
\begin{aligned}
\omega_{w}\left(x_{n}\right):= & \left\{x \in H: x_{n_{i}} \rightarrow x\right. \\
& \text { for some subsequence } \left.\left\{x_{n_{i}}\right\} \text { of }\left\{x_{n}\right\}\right\} .
\end{aligned}
$$


Recall that a mapping $A: C \rightarrow H$ is called

(i) monotone if

$$
\langle A x-A y, x-y\rangle \geq 0, \quad \forall x, y \in C,
$$

(ii) $\eta$-strongly monotone if there exists a constant $\eta>0$ such that

$$
\langle A x-A y, x-y\rangle \geq \eta\|x-y\|^{2}, \quad \forall x, y \in C,
$$

(iii) $\alpha$-inverse-strongly monotone if there exists a constant $\alpha>0$ such that

$$
\langle A x-A y, x-y\rangle \geq \alpha\|A x-A y\|^{2}, \quad \forall x, y \in C .
$$

It is obvious that if $A$ is $\alpha$-inverse-strongly monotone, then $A$ is monotone and $(1 / \alpha)$-Lipschitz continuous. Moreover, we also have that, for all $u, v \in C$ and $\lambda>0$,

$$
\begin{aligned}
\|(I- & \lambda A) u-(I-\lambda A) v \|^{2} \\
& =\|(u-v)-\lambda(A u-A v)\|^{2} \\
& =\|u-v\|^{2}-2 \lambda\langle A u-A v, u-v\rangle+\lambda^{2}\|A u-A v\|^{2} \\
& \leq\|u-v\|^{2}+\lambda(\lambda-2 \alpha)\|A u-A v\|^{2} .
\end{aligned}
$$

So, if $\lambda \leq 2 \alpha$, then $I-\lambda A$ is a nonexpansive mapping from $C$ to $H$.

The metric projection from $H$ onto $C$ is the mapping $P_{C}$ : $H \rightarrow C$ which assigns to each point $x \in H$ the unique point $P_{C} x \in C$ satisfying the property

$$
\left\|x-P_{C} x\right\|=\inf _{y \in C}\|x-y\|=: d(x, C) .
$$

Some important properties of projections are gathered in the following proposition.

Proposition 1. For given $x \in H$ and $z \in C$ :

(i) $z=P_{C} x \Leftrightarrow\langle x-z, y-z\rangle \leq 0$, for all $y \in C$;

(ii) $z=P_{C} x \Leftrightarrow\|x-z\|^{2} \leq\|x-y\|^{2}-\|y-z\|^{2}$, for all $y \in C$;

(iii) $\left\langle P_{C} x-P_{C} y, x-y\right\rangle \geq\left\|P_{C} x-P_{C} y\right\|^{2}$, for all $y \in H$.

Consequently, $P_{C}$ is nonexpansive and monotone.

Definition 2. A mapping $T: H \rightarrow H$ is said to be

(a) nonexpansive if

$$
\|T x-T y\| \leq\|x-y\|, \quad \forall x, y \in H,
$$

(b) firmly nonexpansive if $2 T-I$ is nonexpansive or equivalently if $T$ is 1-inverse-strongly monotone (1ism), then

$$
\langle x-y, T x-T y\rangle \geq\|T x-T y\|^{2}, \quad \forall x, y \in H ;
$$

alternatively, $T$ is firmly nonexpansive if and only if $T$ can be expressed as

$$
T=\frac{1}{2}(I+S),
$$

where $S: H \rightarrow H$ is nonexpansive; projections are firmly nonexpansive.

It can be easily seen that if $T$ is nonexpansive, then $I-T$ is monotone and a projection $P_{C}$ is 1 -ism. The inverse-strongly monotone (also referred to as cocoercive) operators have been applied widely in solving practical problems in various fields.

Definition 3. A mapping $T: H \rightarrow H$ is said to be an averaged mapping if it can be written as the average of the identity $I$ and a nonexpansive mapping; that is,

$$
T \equiv(1-\alpha) I+\alpha S,
$$

where $\alpha \in(0,1)$ and $S: H \rightarrow H$ is nonexpansive. More precisely, when the last equality holds, we say that $T$ is $\alpha$ averaged. Thus firmly nonexpansive mappings (in particular, projections) are (1/2)-averaged mappings.

Proposition 4 (see [37]). Let $T: H \rightarrow H$ be a given mapping.

(i) $T$ is nonexpansive if and only if the complement $I-T$ is $(1 / 2)-i s m$.

(ii) If $T$ is $\nu$-ism, then for $\gamma>0, \gamma T$ is $(\nu / \gamma)$-ism.

(iii) $T$ is averaged if and only if the complement $I-T$ is $\nu$-ism for some $\nu>1 / 2$. Indeed, for $\alpha \in(0,1), T$ is $\alpha$-averaged if and only if $I-T$ is $(1 / 2 \alpha)$-ism.

Proposition 5 (see $[37,38]$ ). Let $S, T, V: H \rightarrow H$ be given operators.

(i) If $T=(1-\alpha) S+\alpha V$ for some $\alpha \in(0,1)$ and if $S$ is averaged and $V$ is nonexpansive, then $T$ is averaged.

(ii) $T$ is firmly nonexpansive if and only if the complement $I-T$ is firmly nonexpansive.

(iii) If $T=(1-\alpha) S+\alpha V$ for some $\alpha \in(0,1)$ and if $S$ is firmly nonexpansive and $V$ is nonexpansive, then $T$ is averaged.

(iv) The composite of finitely many averaged mappings is averaged. That is, if each of the mappings $\left\{T_{i}\right\}_{i=1}^{N}$ is averaged, then so is the composite $T_{1} \cdots T_{N}$. In particular, if $T_{1}$ is $\alpha_{1}$-averaged and $T_{2}$ is $\alpha_{2}$-averaged, where $\alpha_{1}, \alpha_{2} \in(0,1)$, then the composite $T_{1} T_{2}$ is $\alpha$ averaged, where $\alpha=\alpha_{1}+\alpha_{2}-\alpha_{1} \alpha_{2}$.

(v) If the mappings $\left\{T_{i}\right\}_{i=1}^{N}$ are averaged and have a common fixed point, then

$$
\bigcap_{i=1}^{N} \operatorname{Fix}\left(T_{i}\right)=\operatorname{Fix}\left(T_{1} \cdots T_{N}\right) .
$$


The notation $\operatorname{Fix}(T)$ denotes the set of all fixed points of the mapping $T$; that is, $\operatorname{Fix}(T)=\{x \in H: T x=x\}$.

Next we list some elementary conclusions for the MEP.

Proposition 6 (see [25]). Assume that $\Theta: C \times C \rightarrow \mathbf{R}$ satisfies (A1)-(A4) and let $\varphi: C \rightarrow \mathbf{R}$ be a proper lower semicontinuous and convex function. Assume that either (B1) or (B2) holds. For $r>0$ and $x \in H$, define a mapping $T_{r}^{(\Theta, \varphi)}: H \rightarrow C$ as follows:

$$
\begin{array}{r}
T_{r}^{(\Theta, \varphi)}(x)=\{z \in C: \Theta(z, y)+\varphi(y)-\varphi(z) \\
\left.+\frac{1}{r}\langle y-z, z-x\rangle \geq 0, \forall y \in C\right\}
\end{array}
$$

for all $x \in H$. Then the following hold:

(i) for each $x \in H, T_{r}^{(\Theta, \varphi)}(x)$ is nonempty and singlevalued;

(ii) $T_{r}^{(\Theta, \varphi)}$ is firmly nonexpansive; that is, for any $x, y \in H$,

$\left\|T_{r}^{(\Theta, \varphi)} x-T_{r}^{(\Theta, \varphi)} y\right\|^{2} \leq\left\langle T_{r}^{(\Theta, \varphi)} x-T_{r}^{(\Theta, \varphi)} y, x-y\right\rangle ;$

(iii) $\operatorname{Fix}\left(T_{r}^{(\Theta, \varphi)}\right)=\operatorname{MEP}(\Theta, \varphi)$;

(iv) $\operatorname{MEP}(\Theta, \varphi)$ is closed and convex;

(v) $\left\|T_{s}^{(\Theta, \varphi)} x-T_{t}^{(\Theta, \varphi)} x\right\|^{2} \leq((s-t) / s)\left\langle T_{s}^{(\Theta, \varphi)} x-T_{t}^{(\Theta, \varphi)} x\right.$, $\left.T_{s}^{(\Theta, \varphi)} x-x\right\rangle$ for all $s, t>0$ and $x \in H$.

We need some facts and tools in a real Hilbert space $H$ which are listed as lemmas below.

Lemma 7. Let $X$ be a real inner product space. Then there holds the following inequality:

$$
\|x+y\|^{2} \leq\|x\|^{2}+2\langle y, x+y\rangle, \quad \forall x, y \in X
$$

Lemma 8. Let $A: C \rightarrow H$ be a monotone mapping. In the context of the variational inequality problem, the characterization of the projection (see Proposition 1(i)) implies that

$$
u \in V I(C, A) \Longleftrightarrow u=P_{C}(u-\lambda A u), \quad \lambda>0 .
$$

Lemma 9 (see [39, Demiclosedness principle]). Let $C$ be a nonempty closed convex subset of a real Hilbert space H. Let $T$ be a nonexpansive self-mapping on $C$. Then $I-T$ is demiclosed. That is, whenever $\left\{x_{n}\right\}$ is a sequence in $C$ weakly converging to some $x \in C$ and the sequence $\left\{(I-T) x_{n}\right\}$ strongly converges to some $y$, it follows that $(I-T) x=y$. Here $I$ is the identity operator of $H$.

Let $\left\{S_{n}\right\}_{n=1}^{\infty}$ be an infinite family of nonexpansive mappings on $H$ and let $\left\{\lambda_{n}\right\}_{n=1}^{\infty}$ be a sequence of nonnegative numbers in $[0,1]$. For any $n \geq 1$, define a mapping $W_{n}$ on $H$ as follows:

$$
\begin{aligned}
& U_{n, n+1}=I \text {, } \\
& U_{n, n}=\lambda_{n} S_{n} U_{n, n+1}+\left(1-\lambda_{n}\right) I, \\
& U_{n, n-1}=\lambda_{n-1} S_{n-1} U_{n, n}+\left(1-\lambda_{n-1}\right) I \text {, } \\
& U_{n, k}=\lambda_{k} S_{k} U_{n, k+1}+\left(1-\lambda_{k}\right) I, \\
& U_{n, k-1}=\lambda_{k-1} S_{k-1} U_{n, k}+\left(1-\lambda_{k-1}\right) I \text {, } \\
& U_{n, 2}=\lambda_{2} S_{2} U_{n, 3}+\left(1-\lambda_{2}\right) I, \\
& W_{n}=U_{n, 1}=\lambda_{1} S_{1} U_{n, 2}+\left(1-\lambda_{1}\right) I \text {. }
\end{aligned}
$$

Such a mapping $W_{n}$ is called the $W$-mapping generated by $S_{n}, S_{n-1}, \ldots, S_{1}$ and $\lambda_{n}, \lambda_{n-1}, \ldots, \lambda_{1}$.

Lemma 10 (see [40, Lemma 3.2]). Let $C$ be a nonempty closed convex subset of a real Hilbert space $H$. Let $\left\{S_{n}\right\}_{n=1}^{\infty}$ be a sequence of nonexpansive self-mappings on $C$ such that $\cap_{n=1}^{\infty} \operatorname{Fix}\left(S_{n}\right) \neq \emptyset$ and let $\left\{\lambda_{n}\right\}$ be a sequence in $(0, b]$ for some $b \in(0,1)$. Then, for every $x \in C$ and $k \geq 1$, the limit $\lim _{n \rightarrow \infty} U_{n, k} x$ exists where $U_{n, k}$ is defined as in (41).

Remark 11 (see [41, Remark 3.1]). It can be known from Lemma 10 that if $D$ is a nonempty bounded subset of $C$, then for $\epsilon>0$ there exists $n_{0} \geq k$ such that for all $n>n_{0}$

$$
\sup _{x \in D}\left\|U_{n, k} x-U_{k} x\right\| \leq \epsilon .
$$

Remark 12 (see [41, Remark 3.2]). Utilizing Lemma 10, we define a mapping $W: C \rightarrow C$ as follows:

$$
W x=\lim _{n \rightarrow \infty} W_{n} x=\lim _{n \rightarrow \infty} U_{n, 1} x, \quad \forall x \in C .
$$

Such a $W$ is called the $W$-mapping generated by $S_{1}, S_{2}, \ldots$ and $\lambda_{1}, \lambda_{2}, \ldots$.. Since $W_{n}$ is nonexpansive, $W: C \rightarrow C$ is also nonexpansive. If $\left\{x_{n}\right\}$ is a bounded sequence in $C$, then it is clear from Remark 11 that

$$
\lim _{n \rightarrow \infty}\left\|W_{n} x_{n}-W x_{n}\right\|=0
$$

Lemma 13 (see [40, Lemma 3.3]). Let $C$ be a nonempty closed convex subset of a real Hilbert space $H$. Let $\left\{S_{n}\right\}_{n=1}^{\infty}$ be a sequence of nonexpansive self-mappings on $C$ such that $\cap_{n=1}^{\infty} \operatorname{Fix}\left(S_{n}\right) \neq \emptyset$, and let $\left\{\lambda_{n}\right\}$ be a sequence in $(0, b]$ for some $b \in(0,1)$. Then, $\operatorname{Fix}(W)=\cap_{n=1}^{\infty} \operatorname{Fix}\left(S_{n}\right)$.

The following lemma can be easily proven, and therefore, we omit the proof. 
Lemma 14. Let $V: H \rightarrow H$ be an l-Lipschitzian mapping with constant $l \geq 0$ and let $F: H \rightarrow H$ be $a \kappa-$ Lipschitzian and $\eta$-strongly monotone operator with positive constants $\kappa, \eta>0$. Then for $0 \leq \gamma l<\mu \eta$,

$$
\begin{aligned}
& \langle(\mu F-\gamma V) x-(\mu F-\gamma V) y, x-y\rangle \\
& \geq(\mu \eta-\gamma l)\|x-y\|^{2}, \quad \forall x, y \in H .
\end{aligned}
$$

That is, $\mu F-\gamma V$ is strongly monotone with the constant $\mu \eta-\gamma l>$ 0 .

Let $C$ be a nonempty closed convex subset of a real Hilbert space $H$. We introduce some notations. Let $\lambda$ be a number in $(0,1]$ and let $\mu>0$. Associating with a nonexpansive mapping $T: C \rightarrow H$, we define the mapping $T^{\lambda}: C \rightarrow H$ by

$$
T^{\lambda} x:=T x-\lambda \mu F(T x), \quad \forall x \in C,
$$

where $F: H \rightarrow H$ is an operator such that, for some positive constants $\kappa, \eta>0, F$ is $\kappa$-Lipschitzian and $\eta$-strongly monotone on $H$; that is, $F$ satisfies the following conditions:

$$
\begin{gathered}
\|F x-F y\| \leq \kappa\|x-y\|, \\
\langle F x-F y, x-y\rangle \geq \eta\|x-y\|^{2}
\end{gathered}
$$

for all $x, y \in H$.

Lemma 15 (see [42, Lemma 3.1]). $T^{\lambda}$ is a contraction provided that $0<\mu<2 \eta / \kappa^{2}$; that is,

$$
\left\|T^{\lambda} x-T^{\lambda} y\right\| \leq(1-\lambda \tau)\|x-y\|, \quad \forall x, y \in C,
$$

where $\tau=1-\sqrt{1-\mu\left(2 \eta-\mu \kappa^{2}\right)} \in(0,1]$.

Lemma 16 (see [42]). Let $\left\{s_{n}\right\}$ be a sequence of nonnegative numbers satisfying the conditions

$$
s_{n+1} \leq\left(1-\alpha_{n}\right) s_{n}+\alpha_{n} \beta_{n}, \quad \forall n \geq 1,
$$

where $\left\{\alpha_{n}\right\}$ and $\left\{\beta_{n}\right\}$ are sequences of real numbers such that

(i) $\left\{\alpha_{n}\right\} \subset[0,1]$ and $\sum_{n=1}^{\infty} \alpha_{n}=\infty$ or equivalently

$$
\prod_{n=1}^{\infty}\left(1-\alpha_{n}\right):=\lim _{n \rightarrow \infty} \prod_{k=1}^{n}\left(1-\alpha_{k}\right)=0 ;
$$

(ii) $\lim \sup _{n \rightarrow \infty} \beta_{n} \leq 0$, or $\sum_{n=1}^{\infty}\left|\alpha_{n} \beta_{n}\right|<\infty$.

Then $\lim _{n \rightarrow \infty} s_{n}=0$.

Lemma 17 (see [39]). Let $H$ be a real Hilbert space. Then the following hold:

(a) $\|x-y\|^{2}=\|x\|^{2}-\|y\|^{2}-2\langle x-y, y\rangle$ for all $x, y \in H$;

(b) $\|\lambda x+\mu y\|^{2}=\lambda\|x\|^{2}+\mu\|y\|^{2}-\lambda \mu\|x-y\|^{2}$ for all $x, y \in$ $H$ and $\lambda, \mu \in[0,1]$ with $\lambda+\mu=1$;

(c) if $\left\{x_{n}\right\}$ is a sequence in $H$ such that $x_{n} \rightarrow x$, it follows that

$$
\limsup _{n \rightarrow \infty}\left\|x_{n}-y\right\|^{2}=\limsup _{n \rightarrow \infty}\left\|x_{n}-x\right\|^{2}+\|x-y\|^{2} \text {, }
$$

$\forall y \in H$.
A set-valued mapping $\widetilde{T}: H \rightarrow 2^{H}$ is called monotone if for all $x, y \in H, f \in \widetilde{T} x$ and $g \in \widetilde{T} y$ imply that $\langle x-y, f-$ $g\rangle \geq 0$. A monotone set-valued mapping $\widetilde{T}: H \rightarrow 2^{H}$ is called maximal if its graph Gph $(\widetilde{T})$ is not properly contained in the graph of any other monotone set-valued mapping. It is known that a monotone set-valued mapping $\widetilde{T}: H \rightarrow 2^{H}$ is maximal if and only iffor $(x, f) \in H \times H,\langle x-y, f-g\rangle \geq 0$ for every $(y, g) \in G p h(\widetilde{T})$ implies that $f \in \widetilde{T} x$. Let $A: C \rightarrow H$ be a monotone and Lipschitz continuous mapping and let $N_{C} v$ be the normal cone to $C$ at $v \in C$, that is,

$$
N_{C} v=\{w \in H:\langle v-u, w\rangle \geq 0, \forall u \in C\}
$$

Define

$$
\widetilde{T} v= \begin{cases}A v+N_{C} v, & \text { if } v \in C, \\ \emptyset, & \text { if } v \notin C .\end{cases}
$$

Lemma 18 (see [43]). Let $A: C \rightarrow H$ be a monotone mapping. Then there hold the following statements:

(i) $\widetilde{T}$ is maximal monotone;

(ii) $v \in \widetilde{T}^{-1} 0 \Leftrightarrow v \in \operatorname{VI}(C, A)$.

\section{Strong Convergence Criteria for the THVI and HFPP}

In this section, we will introduce and analyze an iterative algorithm for finding a solution of the THVI (24) with constraints of several problems: the finitely many GMEPs, the finitely many VIPs, GSVI (11), and CMP (14) in a real Hilbert space. This algorithm is based on the Korpelevich's extragradient method, the viscosity approximation method, the hybrid steepest-descent method, and the averaged mapping approach to the GPA. We prove the strong convergence of the proposed algorithm to a unique solution of THVI (24) under suitable conditions. In addition, we also consider the application of the proposed algorithm to solve a hierarchical fixed point problem with the same constraints.

Theorem 19. Let $C$ be a nonempty closed convex subset of a real Hilbert space $H$. Let $M, N$ be two integers. Let $f: C \rightarrow \mathbf{R}$ be a convex functional with L-Lipschitz continuous gradient $\nabla f$. Let $\Theta_{k}$ be a bifunction from $C \times C$ to $\mathbf{R}$ satisfying (A1)$\left(\right.$ A4) and let $\varphi_{k}: C \rightarrow \mathbf{R} \cup\{+\infty\}$ be a proper lower semicontinuous and convex function, where $k \in\{1,2, \ldots, M\}$. Let $B_{k}, A_{i}: H \rightarrow H$ and $F_{j}: C \rightarrow H$ be $\mu_{k}$-inverse-strongly monotone, $\eta_{i}$-inverse-strongly monotone, and $\zeta_{j}$-inverse-strongly monotone, respectively, where $k \epsilon$ $\{1,2, \ldots, M\}, i \in\{1,2, \ldots, N\}$, and $j \in\{1,2\}$. Let $S: H \rightarrow$ $H$ be a nonexpansive mapping, let $\left\{S_{n}\right\}_{n=1}^{\infty}$ be a sequence of nonexpansive mappings on $H$, and let $\left\{\lambda_{n}\right\}$ be a sequence in $(0, b]$ for some $b \in(0,1)$. Let $F: H \rightarrow H$ be $a \kappa-$ Lipschitzian and $\eta$-strongly monotone operator with positive constants $\kappa, \eta>0$. Let $V: H \rightarrow H$ be an l-Lipschitzian mapping with the constant $l \geq 0$. Let $0<\mu<2 \eta / \kappa^{2}, 0<\gamma \leq \tau$, and $0 \leq \gamma l<\tau$, where $\tau=1-\sqrt{1-\mu\left(2 \eta-\mu \kappa^{2}\right)}$. Assume that 
either (B1) or (B2) holds. For arbitrarily given $x_{1} \in H$, let $\left\{x_{n}\right\}$ be a sequence generated by

$$
\begin{aligned}
u_{n}= & T_{r_{M, n}}^{\left(\Theta_{M}, \varphi_{M}\right)}\left(I-r_{M, n} B_{M}\right) T_{r_{M-1, n}}^{\left(\Theta_{M-1}, \varphi_{M-1}\right)} \\
& \times\left(I-r_{M-1, n} B_{M-1}\right) \cdots T_{r_{1, n}}^{\left(\Theta_{1}, \varphi_{1}\right)}\left(I-r_{1, n} B_{1}\right) x_{n}, \\
v_{n}= & P_{C}\left(I-\lambda_{N, n} A_{N}\right) \\
& \times P_{C}\left(I-\lambda_{N-1, n} A_{N-1}\right) \cdots P_{C}\left(I-\lambda_{2, n} A_{2}\right) \\
& \times P_{C}\left(I-\lambda_{1, n} A_{1}\right) u_{n}, \\
& y_{n}=\alpha_{n} \gamma S v_{n}+\left(I-\alpha_{n} \mu F\right) W_{n} G v_{n}, \\
x_{n+1} & =s_{n} \gamma V x_{n}+\left(I-s_{n} \mu F\right) T_{n} y_{n}, \quad \forall n \geq 1,
\end{aligned}
$$

where $P_{C}\left(I-\theta_{n} \nabla f\right)=s_{n} I+\left(1-s_{n}\right) T_{n}\left(\right.$ here $T_{n}$ is nonexpansive, $s_{n}=\left(2-\theta_{n} L\right) / 4 \in(0,1 / 2)$ for each $\left.\theta_{n} \in(0,2 / L)\right)$, $\left\{\alpha_{n}\right\} \subset(0,1], \nu_{j} \in\left(0,2 \zeta_{j}\right)$ for $j=1,2$, and $W_{n}$ is the $W$ mapping defined by (41). Suppose that the following conditions are satisfied:

(H1) $s_{n} \in(0,1 / 2)$ for each $\theta_{n} \in(0,2 / L)$, and $\lim _{n \rightarrow \infty} s_{n}=$ $0\left(\Leftrightarrow \lim _{n \rightarrow \infty} \theta_{n}=2 / L\right)$;

(H2) $\sum_{n=1}^{\infty} s_{n}=\infty$ and $\lim _{n \rightarrow \infty}\left(1 / s_{n}\right)\left|1-\left(\alpha_{n-1} / \alpha_{n}\right)\right|=0$;

(H3) $\lim _{n \rightarrow \infty}\left(b^{n} / s_{n} \alpha_{n}\right)=0, \lim _{n \rightarrow \infty}\left(1 / s_{n}\right) \mid\left(1 / \alpha_{n}\right)-$ $\left(1 / \alpha_{n-1}\right) \mid=0$, and $\lim _{n \rightarrow \infty}\left(1 / \alpha_{n}\right)\left|1-\left(s_{n-1} / s_{n}\right)\right|=0 ;$

(H4) $\lim _{n \rightarrow \infty}\left(\alpha_{n} / s_{n}\right)=0$;

(H5) $\left\{\lambda_{i, n}\right\} \subset\left[a_{i}, b_{i}\right] \subset\left(0,2 \eta_{i}\right)$ and $\lim _{n \rightarrow \infty}\left(\mid \lambda_{i, n}-\right.$ $\left.\lambda_{i, n-1} \mid / s_{n} \alpha_{n}\right)=0$ for all $i \in\{1,2, \ldots, N\}$;

(H6) $\left\{r_{k, n}\right\} \subset\left[e_{k}, f_{k}\right] \subset\left(0,2 \mu_{k}\right)$ and $\lim _{n \rightarrow \infty}\left(\mid r_{k, n}-\right.$ $\left.r_{k, n-1} \mid / s_{n} \alpha_{n}\right)=0$ for all $k \in\{1,2, \ldots, M\}$.

Then there hold the following:

(i) $\lim _{n \rightarrow \infty}\left(\left\|x_{n+1}-x_{n}\right\| / \alpha_{n}\right)=0$;

(ii) $\omega_{w}\left(x_{n}\right) \subset \Omega$ provided $\lim _{n \rightarrow \infty}\left\|x_{n}-y_{n}\right\|=0$;

(iii) $\omega_{w}\left(x_{n}\right) \subset \Xi$ provided $\left\|x_{n}-y_{n}\right\|=o\left(\alpha_{n}\right)$ additionally.

Proof. Since $\nabla f$ is $L$-Lipschitzian, it follows that $\nabla f$ is $1 / L$ ism; see [44] (see also [31]). By Proposition 4(ii) we know that for $\theta>0, \theta \nabla f$ is $(1 / \theta L)$-ism. So by Proposition 4(iii) we deduce that $I-\theta \nabla f$ is $(\theta L / 2)$-averaged. Now since the projection $P_{C}$ is $(1 / 2)$-averaged, it is easy to see from Proposition 5(iv) that the composite $P_{C}(I-\theta \nabla f)$ is $((2+$ $\theta L) / 4)$-averaged for $\theta \in(0,2 / L)$. Hence we obtain that for each $n \geq 1, P_{C}\left(I-\theta_{n} \nabla f\right)$ is $\left(\left(2+\theta_{n} L\right) / 4\right)$-averaged for each $\theta_{n} \in(0,2 / L)$. Therefore, we can write

$$
\begin{aligned}
P_{C}\left(I-\theta_{n} \nabla f\right) & =\frac{2-\theta_{n} L}{4} I+\frac{2+\theta_{n} L}{4} T_{n} \\
& =s_{n} I+\left(1-s_{n}\right) T_{n},
\end{aligned}
$$

where $T_{n}$ is nonexpansive and $s_{n}:=s_{n}\left(\theta_{n}\right)=\left(2-\theta_{n} L\right) / 4 \epsilon$ $(0,1 / 2)$ for each $\theta_{n} \in(0,2 / L)$. It is clear that

$$
\theta_{n} \longrightarrow \frac{2}{L} \Longleftrightarrow s_{n} \longrightarrow 0
$$

Put

$$
\begin{aligned}
\Delta_{n}^{k}= & T_{r_{k, n}}^{\left(\Theta_{k}, \varphi_{k}\right)}\left(I-r_{k, n} B_{k}\right) T_{r_{k-1, n}}^{\left(\Theta_{k-1}, \varphi_{k-1}\right)} \\
& \times\left(I-r_{k-1, n} B_{k-1}\right) \cdots T_{r_{1, n}}^{\left(\Theta_{1}, \varphi_{1}\right)}\left(I-r_{1, n} B_{1}\right) x_{n}
\end{aligned}
$$

for all $k \in\{1,2, \ldots, M\}$ and $n \geq 1$,

$$
\begin{aligned}
\Lambda_{n}^{i}= & P_{C}\left(I-\lambda_{i, n} B_{i}\right) \\
& \times P_{C}\left(I-\lambda_{i-1, n} B_{i-1}\right) \cdots P_{C}\left(I-\lambda_{1, n} B_{1}\right)
\end{aligned}
$$

for all $i \in\{1,2, \ldots, N\}, \Delta_{n}^{0}=I$ and $\Lambda_{n}^{0}=I$, where $I$ is the identity mapping on $H$. Then we have $u_{n}=\Delta_{n}^{M} x_{n}$ and $v_{n}=$ $\Lambda_{n}^{N} u_{n}$

We divide the rest of the proof into several steps.

Step 1. Let us show that $\left\{x_{n}\right\}$ is bounded. Indeed, taking into account the assumption $\Xi \neq \emptyset$ in Problem 2, we know that $\Omega \neq \emptyset$. By (H4), we may assume, without loss of generality, that $\alpha_{n} \leq s_{n}$ for all $n \geq 1$. Taking $p \in \Omega$ arbitrarily. Then from (30) and Proposition 6(ii) we have

$$
\begin{array}{ll}
\| u_{n}- & p \| \\
= & \| T_{r_{M, n}^{\left(\Theta_{M}, \varphi_{M}\right)}\left(I-r_{M, n} B_{M}\right) \Delta_{n}^{M-1} x_{n}} \\
& \quad-T_{r_{M, n}^{\left(\Theta_{M}, \varphi_{M}\right)}}\left(I-r_{M, n} B_{M}\right) \Delta_{n}^{M-1} p \| \\
\leq & \left\|\left(I-r_{M, n} B_{M}\right) \Delta_{n}^{M-1} x_{n}-\left(I-r_{M, n} B_{M}\right) \Delta_{n}^{M-1} p\right\| \\
\leq & \left\|\Delta_{n}^{M-1} x_{n}-\Delta_{n}^{M-1} p\right\| \\
\vdots & \\
\leq & \left\|\Delta_{n}^{0} x_{n}-\Delta_{n}^{0} p\right\| \\
= & \left\|x_{n}-p\right\| .
\end{array}
$$

Similarly, we have

$$
\begin{aligned}
& \left\|v_{n}-p\right\| \\
& \quad=\left\|P_{C}\left(I-\lambda_{N, n} A_{N}\right) \Lambda_{n}^{N-1} u_{n}-P_{C}\left(I-\lambda_{N, n} A_{N}\right) \Lambda_{n}^{N-1} p\right\| \\
& \quad \leq\left\|\left(I-\lambda_{N, n} A_{N}\right) \Lambda_{n}^{N-1} u_{n}-\left(I-\lambda_{N, n} A_{N}\right) \Lambda_{n}^{N-1} p\right\| \\
& \quad \leq\left\|\Lambda_{n}^{N-1} u_{n}-\Lambda_{n}^{N-1} p\right\| \\
& \quad \vdots \\
& \quad \leq\left\|\Lambda_{n}^{0} u_{n}-\Lambda_{n}^{0} p\right\| \\
& \quad=\left\|u_{n}-p\right\| .
\end{aligned}
$$

Combining (59) and (60), we have

$$
\left\|v_{n}-p\right\| \leq\left\|x_{n}-p\right\| .
$$


Since $p=G p=P_{C}\left(I-v_{1} F_{1}\right) P_{C}\left(I-v_{2} F_{2}\right) p, F_{j}$ is $\zeta_{j}$-inversestrongly monotone for $j=1,2$, and $0 \leq v_{j} \leq 2 \zeta_{j}$ for $j=1,2$, we deduce that, for any $n \geq 1$,

$$
\begin{aligned}
\| G v_{n}- & p \|^{2} \\
= & \| P_{C}\left(I-v_{1} F_{1}\right) P_{C}\left(I-v_{2} F_{2}\right) v_{n} \\
& -P_{C}\left(I-v_{1} F_{1}\right) P_{C}\left(I-v_{2} F_{2}\right) p \|^{2} \\
\leq & \|\left(I-v_{1} F_{1}\right) P_{C}\left(I-v_{2} F_{2}\right) v_{n} \\
& \quad-\left(I-v_{1} F_{1}\right) P_{C}\left(I-v_{2} F_{2}\right) p \|^{2} \\
= & \|\left[P_{C}\left(I-v_{2} F_{2}\right) v_{n}-P_{C}\left(I-v_{2} F_{2}\right) p\right] \\
& \quad-v_{1}\left[F_{1} P_{C}\left(I-v_{2} F_{2}\right) v_{n}-F_{1} P_{C}\left(I-v_{2} F_{2}\right) p\right] \|^{2} \\
\leq & \left\|P_{C}\left(I-v_{2} F_{2}\right) v_{n}-P_{C}\left(I-v_{2} F_{2}\right) p\right\|^{2} \\
& +v_{1}\left(v_{1}-2 \zeta_{1}\right) \| F_{1} P_{C}\left(I-v_{2} F_{2}\right) v_{n} \\
& \quad-F_{1} P_{C}\left(I-v_{2} F_{2}\right) p \|^{2} \\
\leq & \left\|P_{C}\left(I-v_{2} F_{2}\right) v_{n}-P_{C}\left(I-v_{2} F_{2}\right) p\right\|^{2} \\
\leq & \left\|\left(I-v_{2} F_{2}\right) v_{n}-\left(I-v_{2} F_{2}\right) p\right\|^{2} \\
= & \left\|\left(v_{n}-p\right)-v_{2}\left(F_{2} v_{n}-F_{2} p\right)\right\|^{2} \\
\leq & \left\|v_{n}-p\right\|^{2}+v_{2}\left(v_{2}-2 \zeta_{2}\right)\left\|F_{2} v_{n}-F_{2} p\right\|^{2} \\
\leq & \left\|v_{n}-p\right\|^{2} .
\end{aligned}
$$

Utilizing Lemma 15 and the relation $\alpha_{n} \leq s_{n}$, from (54), (61), and (62), we obtain that

$$
\begin{aligned}
\| y_{n}- & p \| \\
= & \| \alpha_{n} \gamma\left(S v_{n}-S p\right)+\left(I-\alpha_{n} \mu F\right) W_{n} G v_{n} \\
& -\left(I-\alpha_{n} \mu F\right) p+\alpha_{n}(\gamma S-\mu F) p \| \\
\leq & \alpha_{n} \gamma\left\|S v_{n}-S p\right\| \\
& +\left\|\left(I-\alpha_{n} \mu F\right) W_{n} G v_{n}-\left(I-\alpha_{n} \mu F\right) p\right\| \\
& +\alpha_{n}\|(\gamma S-\mu F) p\| \\
\leq & \alpha_{n} \gamma\left\|v_{n}-p\right\|+\left(1-\alpha_{n} \tau\right)\left\|G v_{n}-p\right\| \\
& +\alpha_{n}\|(\gamma S-\mu F) p\| \\
\leq & \alpha_{n} \gamma\left\|v_{n}-p\right\|+\left(1-\alpha_{n} \tau\right)\left\|v_{n}-p\right\| \\
& +\alpha_{n}\|(\gamma S-\mu F) p\| \\
\leq & \alpha_{n} \gamma\left\|x_{n}-p\right\|+\left(1-\alpha_{n} \tau\right)\left\|x_{n}-p\right\| \\
& +\alpha_{n}\|(\gamma S-\mu F) p\| \\
= & \left(1-\alpha_{n}(\tau-\gamma)\right)\left\|x_{n}-p\right\|+\alpha_{n}\|(\gamma S-\mu F) p\|
\end{aligned}
$$

$$
\begin{aligned}
& \leq\left\|x_{n}-p\right\|+\alpha_{n}\|(\gamma S-\mu F) p\| \\
& \leq\left\|x_{n}-p\right\|+s_{n}\|(\gamma S-\mu F) p\|,
\end{aligned}
$$

and hence

$$
\begin{aligned}
\| x_{n+1}- & p \| \\
= & \| s_{n} \gamma\left(V x_{n}-V p\right)+\left(I-s_{n} \mu F\right) T_{n} y_{n} \\
& -\left(I-s_{n} \mu F\right) p+s_{n}(\gamma S-\mu F) p \| \\
\leq & s_{n} \gamma\left\|V x_{n}-V p\right\| \\
& +\left\|\left(I-s_{n} \mu F\right) T_{n} y_{n}-\left(I-s_{n} \mu F\right) p\right\| \\
& +s_{n}\|(\gamma S-\mu F) p\| \\
\leq & s_{n} \gamma l\left\|x_{n}-p\right\|+\left(1-s_{n} \tau\right)\left\|y_{n}-p\right\| \\
& +s_{n}\|(\gamma S-\mu F) p\| \\
\leq & s_{n} \gamma l\left\|x_{n}-p\right\| \\
& +\left(1-s_{n} \tau\right)\left(\left\|x_{n}-p\right\|+s_{n}\|(\gamma S-\mu F) p\|\right) \\
& +s_{n}\|(\gamma S-\mu F) p\| \\
\leq & s_{n} \gamma l\left\|x_{n}-p\right\|+\left(1-s_{n} \tau\right)\left\|x_{n}-p\right\| \\
& +s_{n}\|(\gamma S-\mu F) p\|+s_{n}\|(\gamma S-\mu F) p\| \\
= & \left(1-s_{n}(\tau-\gamma l)\right)\left\|x_{n}-p\right\| \\
& +s_{n}(\|(\gamma S-\mu F) p\|+\|(\gamma S-\mu F) p\|) \\
= & \left(1-s_{n}(\tau-\gamma l)\right)\left\|x_{n}-p\right\| \\
& +s_{n}(\tau-\gamma l) \frac{\|(\gamma S-\mu F) p\|+\|(\gamma S-\mu F) p\|}{\tau-\gamma l} \\
\leq & \max \left\{\left\|x_{n}-p\right\|, \frac{\|(\gamma S-\mu F) p\|+\|(\gamma S-\mu F) p\|}{\tau-\gamma l}\right\} .
\end{aligned}
$$

By induction, we get

$$
\begin{aligned}
& \left\|x_{n}-p\right\| \\
& \quad \leq \max \left\{\left\|x_{1}-p\right\|, \frac{\|(\gamma S-\mu F) p\|+\|(\gamma S-\mu F) p\|}{\tau-\gamma l}\right\}, \\
& \forall n \geq 1 .
\end{aligned}
$$

Hence $\left\{x_{n}\right\}$ is bounded and so are the sequences $\left\{u_{n}\right\},\left\{v_{n}\right\},\left\{y_{n}\right\}$.

Step 2. Let us show that $\lim _{n \rightarrow \infty}\left(\left\|x_{n+1}-x_{n}\right\| / \alpha_{n}\right)=0$. 
Indeed, taking into account the $(1 / L)$-inverse-strong monotonicity of $\nabla f$, we know that $P_{C}\left(I-\theta_{n} \nabla f\right)$ is nonexpansive for $\theta_{n} \in(0,2 / L)$. Hence it follows that for any given $p \in \Omega$,

$$
\begin{aligned}
\| P_{C} & \left(I-\theta_{n+1} \nabla f\right) y_{n} \| \\
& \leq\left\|P_{C}\left(I-\theta_{n+1} \nabla f\right) y_{n}-p\right\|+\|p\| \\
& =\left\|P_{C}\left(I-\theta_{n+1} \nabla f\right) y_{n}-P_{C}\left(I-\theta_{n+1} \nabla f\right) p\right\|+\|p\| \\
& \leq\left\|y_{n}-p\right\|+\|p\| \\
& \leq\left\|y_{n}\right\|+2\|p\| .
\end{aligned}
$$

This together with the boundedness of $\left\{y_{n}\right\}$ implies that $\left\{P_{C}\left(I-\lambda_{n+1} \nabla f\right) y_{n}\right\}$ is bounded. Also, observe that

$$
\begin{aligned}
\left\|T_{n+1} y_{n}-T_{n} y_{n}\right\| & \| \frac{4 P_{C}\left(I-\theta_{n+1} \nabla f\right)-\left(2-\theta_{n+1} L\right) I}{2+\theta_{n+1} L} y_{n} \\
& \quad-\frac{4 P_{C}\left(I-\theta_{n} \nabla f\right)-\left(2-\theta_{n} L\right) I}{2+\theta_{n} L} y_{n} \| \\
\leq & \left\|\frac{4 P_{C}\left(I-\theta_{n+1} \nabla f\right)}{2+\theta_{n+1} L} y_{n}-\frac{4 P_{C}\left(I-\theta_{n} \nabla f\right)}{2+\theta_{n} L} y_{n}\right\| \\
+ & \left\|\frac{2-\theta_{n} L}{2+\theta_{n} L} y_{n}-\frac{2-\theta_{n+1} L}{2+\theta_{n+1} L} y_{n}\right\| \\
= & \|\left(4\left(2+\theta_{n} L\right) P_{C}\left(I-\theta_{n+1} \nabla f\right) y_{n}\right. \\
& \left.-4\left(2+\theta_{n+1} L\right) P_{C}\left(I-\theta_{n} \nabla f\right) G y_{n}\right) \\
& \times\left(\left(2+\theta_{n+1} L\right)\left(2+\theta_{n} L\right)\right)^{-1} \| \\
& +\frac{4 L\left|\theta_{n+1}-\theta_{n}\right|}{\left(2+\theta_{n+1} L\right)\left(2+\theta_{n} L\right)}\left\|y_{n}\right\| \\
= & \|\left(4 L\left(\theta_{n}-\theta_{n+1}\right) P_{C}\left(I-\theta_{n+1} \nabla f\right) y_{n}+4\left(2+\theta_{n+1} L\right)\right. \\
& \left.\quad \times\left(P_{C}\left(I-\theta_{n+1} \nabla f\right) y_{n}-P_{C}\left(I-\theta_{n} \nabla f\right) y_{n}\right)\right) \\
& \times\left(\left(2+\theta_{n+1} L\right)\left(2+\theta_{n} L\right)\right)^{-1} \| \\
+ & \frac{4 L\left|\theta_{n}-\theta_{n+1}\right|\left\|P_{C}\left(I-\theta_{n+1} \nabla f\right) y_{n}\right\|}{\left(2+\theta_{n+1} L\right)\left(2+\theta_{n} L\right)} \\
& \quad\left(\left(4\left(2+\theta_{n+1} L\right)\right.\right. \\
& \left.\times\left\|P_{C}\left(I-\theta_{n+1} \nabla f\right) y_{n}-P_{C}\left(I-\theta_{n} \nabla f\right) y_{n}\right\|\right)
\end{aligned}
$$

$$
\begin{aligned}
& \left.\quad \times\left(\left(2+\theta_{n+1} L\right)\left(2+\theta_{n} L\right)\right)^{-1}\right) \\
& +\frac{4 L\left|\theta_{n+1}-\theta_{n}\right|}{\left(2+\theta_{n+1} L\right)\left(2+\theta_{n} L\right)}\left\|y_{n}\right\| \\
& \leq\left|\theta_{n+1}-\theta_{n}\right| \\
& \quad \times\left[L\left\|P_{C}\left(I-\theta_{n+1} \nabla f\right) y_{n}\right\|+4\left\|\nabla f\left(y_{n}\right)\right\|+L\left\|y_{n}\right\|\right] \\
& \leq \widetilde{M}\left|\theta_{n+1}-\theta_{n}\right|,
\end{aligned}
$$

where $\sup _{n \geq 1}\left\{L\left\|P_{C}\left(I-\theta_{n+1} \nabla f\right) y_{n}\right\|+4\left\|\nabla f\left(y_{n}\right)\right\|+L\left\|y_{n}\right\|\right\} \leq \widetilde{M}$ for some $\widetilde{M}>0$. So, by (67), we have that

$$
\begin{aligned}
& \left\|T_{n+1} y_{n+1}-T_{n} y_{n}\right\| \\
& \quad \leq\left\|T_{n+1} y_{n+1}-T_{n+1} y_{n}\right\|+\left\|T_{n+1} y_{n}-T_{n} y_{n}\right\| \\
& \quad \leq\left\|y_{n+1}-y_{n}\right\|+\widetilde{M}\left|\theta_{n+1}-\theta_{n}\right| \\
& \quad \leq\left\|y_{n+1}-y_{n}\right\|+\frac{4 \widetilde{M}}{L}\left|s_{n+1}-s_{n}\right| .
\end{aligned}
$$

Note that

$$
\begin{aligned}
\| v_{n+1}- & v_{n} \| \\
= & \left\|\Lambda_{n+1}^{N} u_{n+1}-\Lambda_{n}^{N} u_{n}\right\| \\
= & \| P_{C}\left(I-\lambda_{N, n+1} A_{N}\right) \Lambda_{n+1}^{N-1} u_{n+1} \\
& -P_{C}\left(I-\lambda_{N, n} A_{N}\right) \Lambda_{n}^{N-1} u_{n} \| \\
\leq & \| P_{C}\left(I-\lambda_{N, n+1} A_{N}\right) \Lambda_{n+1}^{N-1} u_{n+1} \\
& -P_{C}\left(I-\lambda_{N, n} A_{N}\right) \Lambda_{n+1}^{N-1} u_{n+1} \| \\
& +\| P_{C}\left(I-\lambda_{N, n} A_{N}\right) \Lambda_{n+1}^{N-1} u_{n+1} \\
& \quad-P_{C}\left(I-\lambda_{N, n} A_{N}\right) \Lambda_{n}^{N-1} u_{n} \| \\
\leq & \|\left(I-\lambda_{N, n+1} A_{N}\right) \Lambda_{n+1}^{N-1} u_{n+1} \\
& -\left(I-\lambda_{N, n} A_{N}\right) \Lambda_{n+1}^{N-1} u_{n+1} \| \\
& +\|\left(I-\lambda_{N, n} A_{N}\right) \Lambda_{n+1}^{N-1} u_{n+1} \\
& \quad-\left(I-\lambda_{N, n} A_{N}\right) \Lambda_{n}^{N-1} u_{n} \| \\
\leq & \left|\lambda_{N, n+1}-\lambda_{N, n}\right|\left\|A_{N} \Lambda_{n+1}^{N-1} u_{n+1}\right\| \\
& +\left\|\Lambda_{n+1}^{N-1} u_{n+1}-\Lambda_{n}^{N-1} u_{n}\right\| \\
\leq & \left|\lambda_{N, n+1}-\lambda_{N, n}\right|\left\|A_{N} \Lambda_{n+1}^{N-1} u_{n+1}\right\|
\end{aligned}
$$




$$
\begin{aligned}
& \quad+\left|\lambda_{N-1, n+1}-\lambda_{N-1, n}\right|\left\|A_{N-1} \Lambda_{n+1}^{N-2} u_{n+1}\right\| \\
& \quad+\left\|\Lambda_{n+1}^{N-2} u_{n+1}-\Lambda_{n}^{N-2} u_{n}\right\| \\
& \vdots \\
& \leq\left|\lambda_{N, n+1}-\lambda_{N, n}\right|\left\|A_{N} \Lambda_{n+1}^{N-1} u_{n+1}\right\| \\
& \quad+\left|\lambda_{N-1, n+1}-\lambda_{N-1, n}\right|\left\|A_{N-1} \Lambda_{n+1}^{N-2} u_{n+1}\right\| \\
& \quad+\cdots+\left|\lambda_{1, n+1}-\lambda_{1, n}\right|\left\|A_{1} \Lambda_{n+1}^{0} u_{n+1}\right\| \\
& \quad+\left\|\Lambda_{n+1}^{0} u_{n+1}-\Lambda_{n}^{0} u_{n}\right\| \\
& \leq \widetilde{M}_{0} \sum_{i=1}^{N}\left|\lambda_{i, n+1}-\lambda_{i, n}\right|+\left\|u_{n+1}-u_{n}\right\|,
\end{aligned}
$$

where $\sup _{n \geq 1}\left\{\sum_{i=1}^{N}\left\|A_{i} \Lambda_{n+1}^{i-1} u_{n+1}\right\|\right\} \leq \widetilde{M}_{0}$ for some $\widetilde{M}_{0}>0$. Also, utilizing Proposition 6(ii), (v) we deduce that

$$
\begin{aligned}
& \left\|u_{n+1}-u_{n}\right\| \\
& =\left\|\Delta_{n+1}^{M} x_{n+1}-\Delta_{n}^{M} x_{n}\right\| \\
& =\| T_{r_{M, n+1}^{\left(\Theta_{M}, \varphi_{M}\right)}}^{\left(I-r_{M, n+1} B_{M}\right) \Delta_{n+1}^{M-1} x_{n+1}} \\
& -T_{r_{M, n}}^{\left(\Theta_{M}, \varphi_{M}\right)}\left(I-r_{M, n} B_{M}\right) \Delta_{n}^{M-1} x_{n} \| \\
& \leq \| T_{r_{M, n+1}}^{\left(\Theta_{M}, \varphi_{M}\right)}\left(I-r_{M, n+1} B_{M}\right) \Delta_{n+1}^{M-1} x_{n+1} \\
& -T_{r_{M, n}}^{\left(\Theta_{M}, \varphi_{M}\right)}\left(I-r_{M, n} B_{M}\right) \Delta_{n+1}^{M-1} x_{n+1} \|
\end{aligned}
$$

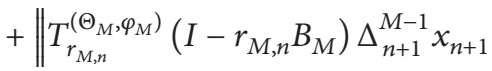

$$
\begin{aligned}
& -T_{r_{M, n}}^{\left(\Theta_{M}, \varphi_{M}\right)}\left(I-r_{M, n} B_{M}\right) \Delta_{n}^{M-1} x_{n} \|
\end{aligned}
$$

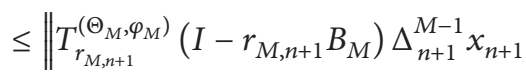

$$
\begin{aligned}
& -T_{r_{M, n}}^{\left(\Theta_{M}, \varphi_{M}\right)}\left(I-r_{M, n+1} B_{M}\right) \Delta_{n+1}^{M-1} x_{n+1} \| \\
& +\| T_{r_{M, n}}^{\left(\Theta_{M}, \varphi_{M}\right)}\left(I-r_{M, n+1} B_{M}\right) \Delta_{n+1}^{M-1} x_{n+1} \\
& -T_{r_{M, n}}^{\left(\Theta_{M}, \varphi_{M}\right)}\left(I-r_{M, n} B_{M}\right) \Delta_{n+1}^{M-1} x_{n+1} \| \\
& +\|\left(I-r_{M, n} B_{M}\right) \Delta_{n+1}^{M-1} x_{n+1} \\
& -\left(I-r_{M, n} B_{M}\right) \Delta_{n}^{M-1} x_{n} \| \\
& \leq \frac{\left|r_{M, n+1}-r_{M, n}\right|}{r_{M, n+1}} \\
& \times \| T_{r_{M, n+1}}^{\left(\Theta_{M}, \varphi_{M}\right)}\left(I-r_{M, n+1} B_{M}\right) \Delta_{n+1}^{M-1} x_{n+1} \\
& -\left(I-r_{M, n+1} B_{M}\right) \Delta_{n+1}^{M-1} x_{n+1} \|
\end{aligned}
$$

$$
\begin{aligned}
& +\left|r_{M, n+1}-r_{M, n}\right|\left\|B_{M} \Delta_{n+1}^{M-1} x_{n+1}\right\| \\
& +\left\|\Delta_{n+1}^{M-1} x_{n+1}-\Delta_{n}^{M-1} x_{n}\right\| \\
& =\left|r_{M, n+1}-r_{M, n}\right| \\
& \quad \times\left[\left\|B_{M} \Delta_{n+1}^{M-1} x_{n+1}\right\|+\frac{1}{r_{M, n+1}}\right. \\
& \quad \times \| T_{r_{M, n+1}^{\left(\Theta_{M}, \varphi_{M}\right)}\left(I-r_{M, n+1} B_{M}\right) \Delta_{n+1}^{M-1} x_{n+1}} \\
& \left.\quad-\left(I-r_{M, n+1} B_{M}\right) \Delta_{n+1}^{M-1} x_{n+1} \|\right] \\
& +\left\|\Delta_{n+1}^{M-1} x_{n+1}-\Delta_{n}^{M-1} x_{n}\right\|
\end{aligned}
$$$$
\leq\left|r_{M, n+1}-r_{M, n}\right|
$$$$
\times\left[\left\|B_{M} \Delta_{n+1}^{M-1} x_{n+1}\right\|+\frac{1}{r_{M, n+1}}\right.
$$

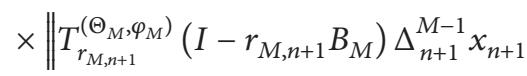$$
\left.-\left(I-r_{M, n+1} B_{M}\right) \Delta_{n+1}^{M-1} x_{n+1} \|\right]
$$$$
+\cdots+\left|r_{1, n+1}-r_{1, n}\right|
$$$$
\times\left[\left\|B_{1} \Delta_{n+1}^{0} x_{n+1}\right\|+\frac{1}{r_{1, n+1}}\right.
$$

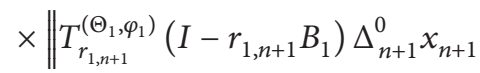$$
\left.-\left(I-r_{1, n+1} B_{1}\right) \Delta_{n+1}^{0} x_{n+1} \|\right]
$$$$
+\left\|\Delta_{n+1}^{0} x_{n+1}-\Delta_{n}^{0} x_{n}\right\|
$$$$
\leq \widetilde{M}_{1} \sum_{k=1}^{M}\left|r_{k, n+1}-r_{k, n}\right|+\left\|x_{n+1}-x_{n}\right\| \text {, }
$$

where $\widetilde{M}_{1}>0$ is a constant such that for each $n \geq 1$

$$
\begin{gathered}
\sum_{k=1}^{M}\left[\left\|B_{k} \Delta_{n+1}^{k-1} x_{n+1}\right\|+\frac{1}{r_{k, n+1}}\right. \\
\times \| T_{r_{k, n+1}^{\left(\Theta_{k}, \varphi_{k}\right)}\left(I-r_{k, n+1} B_{k}\right) \Delta_{n+1}^{k-1} x_{n+1}} \\
\left.\quad-\left(I-r_{k, n+1} B_{k}\right) \Delta_{n+1}^{k-1} x_{n+1} \|\right] \leq \widetilde{M}_{1} .
\end{gathered}
$$


Also, from (54) we have

$$
\begin{aligned}
& y_{n+1}=\alpha_{n+1} \gamma S v_{n+1}+\left(I-\alpha_{n+1} \mu F\right) W_{n+1} G v_{n+1}, \\
& y_{n}=\alpha_{n} \gamma S v_{n}+\left(I-\alpha_{n} \mu F\right) W_{n} G v_{n}, \quad \forall n \geq 1 .
\end{aligned}
$$

Simple calculation shows that

$$
\begin{aligned}
y_{n+1}-y_{n}= & \alpha_{n+1} \gamma\left(S v_{n+1}-S v_{n}\right) \\
& +\left(\alpha_{n+1}-\alpha_{n}\right)\left(\gamma S v_{n}-\mu F W_{n} G v_{n}\right) \\
& +\left(I-\alpha_{n+1} \mu F\right) W_{n+1} G v_{n+1} \\
& -\left(I-\alpha_{n+1} \mu F\right) W_{n} G v_{n} .
\end{aligned}
$$

In the meantime, from (41), since $W_{n}, S_{n}$, and $U_{n, i}$ are all nonexpansive, we have

$$
\begin{aligned}
&\left\|W_{n+1} G v_{n+1}-W_{n} G v_{n}\right\| \\
& \leq\left\|W_{n+1} G v_{n+1}-W_{n+1} G v_{n}\right\| \\
&+\left\|W_{n+1} G v_{n}-W_{n} G v_{n}\right\| \\
& \leq\left\|v_{n+1}-v_{n}\right\|+\left\|W_{n+1} G v_{n}-W_{n} G v_{n}\right\| \\
&=\left\|v_{n+1}-v_{n}\right\| \\
&+\left\|\lambda_{1} T_{1} U_{n+1,2} G v_{n}-\lambda_{1} T_{1} U_{n, 2} G v_{n}\right\| \\
& \leq\left\|v_{n+1}-v_{n}\right\|+\lambda_{1}\left\|U_{n+1,2} G v_{n}-U_{n, 2} G v_{n}\right\| \\
&=\left\|v_{n+1}-v_{n}\right\| \\
&+\lambda_{1}\left\|\lambda_{2} T_{2} U_{n+1,3} G v_{n}-\lambda_{2} T_{2} U_{n, 3} G v_{n}\right\| \\
& \leq\left\|v_{n+1}-v_{n}\right\|+\lambda_{1} \lambda_{2}\left\|U_{n+1,3} G v_{n}-U_{n, 3} G v_{n}\right\| \\
& \vdots \\
& \leq\left\|v_{n+1}-v_{n}\right\|+\lambda_{1} \lambda_{2} \cdots \lambda_{n} \\
& \times\left\|U_{n+1, n+1} G v_{n}-U_{n, n+1} G v_{n}\right\| \\
& \leq\left\|v_{n+1}-v_{n}\right\|+\widetilde{M}_{2} \prod_{i=1}^{n} \lambda_{i},
\end{aligned}
$$

where $\widetilde{M}_{2}$ is a constant such that $\left\|U_{n+1, n+1} G v_{n}\right\|+$ $\left\|U_{n, n+1} G v_{n}\right\| \leq \widetilde{M}_{2}$ for each $n \geq 1$. Therefore, by utilizing Lemma 15, from (69)-(74) and $\left\{\lambda_{n}\right\} \subset(0, b] \subset(0,1)$ it follows that

$$
\begin{aligned}
& \left\|y_{n+1}-y_{n}\right\| \\
& \leq \alpha_{n+1} \gamma\left\|S v_{n+1}-S v_{n}\right\| \\
& \quad+\left|\alpha_{n+1}-\alpha_{n}\right|\left\|\gamma S v_{n}-\mu F W_{n} G v_{n}\right\|
\end{aligned}
$$

$$
\begin{aligned}
& +\|\left(I-\alpha_{n+1} \mu F\right) W_{n+1} G v_{n+1} \\
& -\left(I-\alpha_{n+1} \mu F\right) W_{n} G v_{n} \| \\
& \leq \alpha_{n+1} \gamma\left\|v_{n+1}-v_{n}\right\|+\left|\alpha_{n+1}-\alpha_{n}\right| \\
& \times\left\|\gamma S v_{n}-\mu F W_{n} G v_{n}\right\| \\
& +\left(1-\alpha_{n+1} \tau\right)\left\|W_{n+1} G v_{n+1}-W_{n} G v_{n}\right\| \\
& \leq \alpha_{n+1} \gamma\left\|v_{n+1}-v_{n}\right\|+\left|\alpha_{n+1}-\alpha_{n}\right| \\
& \times\left\|\gamma S v_{n}-\mu F W_{n} G v_{n}\right\| \\
& +\left(1-\alpha_{n+1} \tau\right)\left[\left\|v_{n+1}-v_{n}\right\|+\widetilde{M}_{2} \prod_{i=1}^{n} \lambda_{i}\right] \\
& \leq\left(1-\alpha_{n+1}(\tau-\gamma)\right)\left\|v_{n+1}-v_{n}\right\| \\
& +\left|\alpha_{n+1}-\alpha_{n}\right|\left\|\gamma S v_{n}-\mu F W_{n} G v_{n}\right\|+\widetilde{M}_{2} \prod_{i=1}^{n} \lambda_{i} \\
& \leq\left(1-\alpha_{n+1}(\tau-\gamma)\right) \\
& \times\left[\widetilde{M}_{0} \sum_{i=1}^{N}\left|\lambda_{i, n+1}-\lambda_{i, n}\right|+\left\|u_{n+1}-u_{n}\right\|\right] \\
& +\left|\alpha_{n+1}-\alpha_{n}\right|\left\|\gamma S v_{n}-\mu F W_{n} G v_{n}\right\|+\widetilde{M}_{2} \prod_{i=1}^{n} \lambda_{i} \\
& \leq\left(1-\alpha_{n+1}(\tau-\gamma)\right) \\
& \times\left[\widetilde{M}_{0} \sum_{i=1}^{N}\left|\lambda_{i, n+1}-\lambda_{i, n}\right|\right. \\
& \left.+\widetilde{M}_{1} \sum_{k=1}^{M}\left|r_{k, n+1}-r_{k, n}\right|+\left\|x_{n+1}-x_{n}\right\|\right] \\
& +\left|\alpha_{n+1}-\alpha_{n}\right|\left\|\gamma S v_{n}-\mu F W_{n} G v_{n}\right\|+\widetilde{M}_{2} \prod_{i=1}^{n} \lambda_{i} \\
& \leq\left(1-\alpha_{n+1}(\tau-\gamma)\right)\left\|x_{n+1}-x_{n}\right\| \\
& +\widetilde{M}_{0} \sum_{i=1}^{N}\left|\lambda_{i, n+1}-\lambda_{i, n}\right|+\widetilde{M}_{1} \sum_{k=1}^{M}\left|r_{k, n+1}-r_{k, n}\right| \\
& +\left|\alpha_{n+1}-\alpha_{n}\right|\left\|\gamma S v_{n}-\mu F W_{n} G v_{n}\right\|+\widetilde{M}_{2} \prod_{i=1}^{n} \lambda_{i} .
\end{aligned}
$$

On the other hand, from (54) we have

$$
\begin{aligned}
& x_{n+2}=s_{n+1} \gamma V x_{n+1}+\left(I-s_{n+1} \mu F\right) T_{n+1} y_{n+1}, \\
& x_{n+1}=s_{n} \gamma V x_{n}+\left(I-s_{n} \mu F\right) T_{n} y_{n}, \quad \forall n \geq 1 .
\end{aligned}
$$


The simple calculations show that

$$
\begin{aligned}
x_{n+2}-x_{n+1}= & \left(I-s_{n+1} \mu F\right) T_{n+1} y_{n+1} \\
& -\left(I-s_{n+1} \mu F\right) T_{n} y_{n} \\
& +\left(s_{n+1}-s_{n}\right)\left(\gamma V x_{n}-\mu F T_{n} y_{n}\right) \\
& +s_{n+1} \gamma\left(V x_{n+1}-V x_{n}\right) .
\end{aligned}
$$

Utilizing Lemma 15 we deduce from (68), (75), and (77) that

$$
\begin{aligned}
& \left\|x_{n+2}-x_{n+1}\right\| \\
& \leq\left\|\left(I-s_{n+1} \mu F\right) T_{n+1} y_{n+1}-\left(I-s_{n+1} \mu F\right) T_{n} y_{n}\right\| \\
& +\left|s_{n+1}-s_{n}\right|\left\|\gamma V x_{n}-\mu F T_{n} y_{n}\right\| \\
& +s_{n+1} \gamma\left\|V x_{n+1}-V x_{n}\right\| \\
& \leq\left(1-s_{n+1} \tau\right)\left\|T_{n+1} y_{n+1}-T_{n} y_{n}\right\| \\
& +\left|s_{n+1}-s_{n}\right|\left\|\gamma V x_{n}-\mu F T_{n} y_{n}\right\| \\
& +s_{n+1} \gamma l\left\|x_{n+1}-x_{n}\right\| \\
& \leq\left(1-s_{n+1} \tau\right)\left[\left\|y_{n+1}-y_{n}\right\|+\frac{4 \widetilde{M}}{L}\left|s_{n+1}-s_{n}\right|\right] \\
& +\left|s_{n+1}-s_{n}\right|\left\|\gamma V x_{n}-\mu F T_{n} y_{n}\right\| \\
& +s_{n+1} \gamma l\left\|x_{n+1}-x_{n}\right\| \\
& \leq\left(1-s_{n+1} \tau\right) \\
& \times\left[\left(1-\alpha_{n+1}(\tau-\gamma)\right)\left\|x_{n+1}-x_{n}\right\|\right. \\
& +\widetilde{M}_{0} \sum_{i=1}^{N}\left|\lambda_{i, n+1}-\lambda_{i, n}\right|+\widetilde{M}_{1} \sum_{k=1}^{M}\left|r_{k, n+1}-r_{k, n}\right| \\
& +\left|\alpha_{n+1}-\alpha_{n}\right|\left\|\gamma S v_{n}-\mu F W_{n} G v_{n}\right\| \\
& \left.+\widetilde{M}_{2} \prod_{i=1}^{n} \lambda_{i}+\frac{4 \widetilde{M}}{L}\left|s_{n+1}-s_{n}\right|\right] \\
& +\left|s_{n+1}-s_{n}\right|\left\|\gamma V x_{n}-\mu F T_{n} y_{n}\right\| \\
& +s_{n+1} \gamma l\left\|x_{n+1}-x_{n}\right\| \\
& \leq\left(1-s_{n+1} \tau\right)\left\|x_{n+1}-x_{n}\right\| \\
& +\widetilde{M}_{0} \sum_{i=1}^{N}\left|\lambda_{i, n+1}-\lambda_{i, n}\right|+\widetilde{M}_{1} \sum_{k=1}^{M}\left|r_{k, n+1}-r_{k, n}\right| \\
& +\left|\alpha_{n+1}-\alpha_{n}\right|\left\|\gamma S v_{n}-\mu F W_{n} G v_{n}\right\|
\end{aligned}
$$

$$
\begin{aligned}
& +\widetilde{M}_{2} b^{n}+\left(\frac{4 \widetilde{M}}{L}+\left\|\gamma V x_{n}-\mu F T_{n} y_{n}\right\|\right)\left|s_{n+1}-s_{n}\right| \\
& +s_{n+1} \gamma l\left\|x_{n+1}-x_{n}\right\| \\
= & \left(1-s_{n+1}(\tau-\gamma l)\right)\left\|x_{n+1}-x_{n}\right\| \\
& +\widetilde{M}_{0} \sum_{i=1}^{N}\left|\lambda_{i, n+1}-\lambda_{i, n}\right|+\widetilde{M}_{1} \sum_{k=1}^{M}\left|r_{k, n+1}-r_{k, n}\right| \\
& +\left|\alpha_{n+1}-\alpha_{n}\right|\left\|\gamma S v_{n}-\mu F W_{n} G v_{n}\right\| \\
& +\widetilde{M}_{2} b^{n}+\left(\frac{4 \widetilde{M}}{L}+\left\|\gamma V x_{n}-\mu F T_{n} y_{n}\right\|\right)\left|s_{n+1}-s_{n}\right| \\
\leq & \left(1-s_{n+1}(\tau-\gamma l)\right)\left\|x_{n+1}-x_{n}\right\| \\
& +\widetilde{M}_{3}\left(\sum_{i=1}^{N}\left|\lambda_{i, n+1}-\lambda_{i, n}\right|+\sum_{k=1}^{M}\left|r_{k, n+1}-r_{k, n}\right|\right. \\
& \left.+\left|\alpha_{n+1}-\alpha_{n}\right|+b^{n}+\left|s_{n+1}-s_{n}\right|\right)
\end{aligned}
$$

where $\widetilde{M}_{3}>0$ is a constant such that for each $n \geq 1$

$$
\begin{aligned}
\widetilde{M}_{0}+ & \widetilde{M}_{1}+\widetilde{M}_{2}+\frac{4 \widetilde{M}}{L}+\left\|\gamma V x_{n}-\mu F T_{n} y_{n}\right\| \\
& +\left\|\gamma S v_{n}-\mu F W_{n} G v_{n}\right\| \leq \widetilde{M}_{3} .
\end{aligned}
$$

Therefore,

$$
\begin{aligned}
& \frac{\left\|x_{n+1}-x_{n}\right\|}{\alpha_{n}} \\
& \leq\left(1-s_{n}(\tau-\gamma l)\right) \frac{\left\|x_{n}-x_{n-1}\right\|}{\alpha_{n}} \\
& +\widetilde{M}_{3}\left(\sum_{i=1}^{N} \frac{\left|\lambda_{i, n}-\lambda_{i, n-1}\right|}{\alpha_{n}}+\sum_{k=1}^{M} \frac{\left|r_{k, n}-r_{k, n-1}\right|}{\alpha_{n}}\right. \\
& +\frac{\left|\alpha_{n}-\alpha_{n-1}\right|}{\left.\alpha_{n}+\frac{b^{n-1}}{\alpha_{n}}+\frac{\left|s_{n}-s_{n-1}\right|}{\alpha_{n}}\right)} \\
& +\left(1-s_{n}(\tau-\gamma l)\right) \frac{|| x_{n}-x_{n-1} \|}{\alpha_{n-1}} \\
& \left.+s_{n}(\tau-\gamma l)\right)\left\|x_{n}-x_{n-1}\right\|\left(\frac{1}{\alpha_{n}}-\frac{1}{\alpha_{n-1}}\right) \\
& +\widetilde{M}_{3}\left(\sum_{i=1}^{N} \frac{\left|\lambda_{i, n}-\lambda_{i, n-1}\right|}{\alpha_{n}}+\sum_{k=1}^{M} \frac{\left|r_{k, n}-r_{k, n-1}\right|}{\alpha_{n}}\right. \\
& \left.+\frac{\left|\alpha_{n}-\alpha_{n-1}\right|}{\alpha_{n}}+\frac{b^{n-1}}{\alpha_{n}}+\frac{\left|s_{n}-s_{n-1}\right|}{\alpha_{n}}\right)
\end{aligned}
$$




$$
\begin{aligned}
\leq & \left(1-(\tau-\gamma l) s_{n}\right) \frac{\left\|x_{n}-x_{n-1}\right\|}{\alpha_{n-1}}+(\tau-\gamma l) s_{n} \cdot \frac{1}{\tau-\gamma l} \\
\times & \left\{\left\|x_{n}-x_{n-1}\right\| \frac{1}{s_{n}}\left|\frac{1}{\alpha_{n}}-\frac{1}{\alpha_{n-1}}\right|\right. \\
& +\widetilde{M}_{3}\left(\sum_{i=1}^{N} \frac{\left|\lambda_{i, n}-\lambda_{i, n-1}\right|}{s_{n} \alpha_{n}}+\sum_{k=1}^{M} \frac{\left|r_{k, n}-r_{k, n-1}\right|}{s_{n} \alpha_{n}}\right. \\
\leq & \left.\left.+\frac{\left|\alpha_{n}-\alpha_{n-1}\right|}{s_{n} \alpha_{n}}+\frac{b^{n-1}}{s_{n} \alpha_{n}}+\frac{\left|s_{n}-s_{n-1}\right|}{s_{n} \alpha_{n}}\right)\right\} \\
\times & \left.(\tau-\gamma l) s_{n}\right) \frac{|| x_{n}-x_{n-1} \|}{\alpha_{n-1}}+(\tau-\gamma l) s_{n} \cdot \frac{\widetilde{M}_{4}}{\tau-\gamma l} \\
& +\frac{1}{s_{n}}\left|\frac{1}{\alpha_{n}}-\frac{1}{\alpha_{n-1}}\right| \\
& +\sum_{i=1}^{N} \mid \frac{\lambda_{i, n}-\lambda_{i, n-1} \mid}{s_{n} \alpha_{n}}+\sum_{k=1}^{M} \frac{\left|r_{k, n}-r_{k, n-1}\right|}{s_{n} \alpha_{n}} \\
& \left.+1-\frac{\alpha_{n-1}}{\alpha_{n}}\left|+\frac{1}{\alpha_{n}}\right| 1-\frac{s_{n-1}}{s_{n}} \mid+\frac{b^{n-1}}{s_{n} \alpha_{n}}\right\}
\end{aligned}
$$

where $\widetilde{M}_{3}+\left\|x_{n}-x_{n-1}\right\| \leq \widetilde{M}_{4}$, for all $n>1$ for some $\widetilde{M}_{4}>$ 0 . From (H2), (H3), (H5), and (H6), it follows that $\sum_{n=1}^{\infty}(\tau-$ $\gamma l) s_{n}=\infty$ and

$$
\begin{aligned}
\lim _{n \rightarrow \infty} \frac{\widetilde{M}_{4}}{\tau-\gamma l}\left\{\frac{1}{s_{n}}\left|\frac{1}{\alpha_{n}}-\frac{1}{\alpha_{n-1}}\right|+\sum_{i=1}^{N} \frac{\left|\lambda_{i, n}-\lambda_{i, n-1}\right|}{s_{n} \alpha_{n}}\right. \\
+\sum_{k=1}^{M} \frac{\left|r_{k, n}-r_{k, n-1}\right|}{s_{n} \alpha_{n}}+\frac{1}{s_{n}}\left|1-\frac{\alpha_{n-1}}{\alpha_{n}}\right| \\
\left.+\frac{1}{\alpha_{n}}\left|1-\frac{s_{n-1}}{s_{n}}\right|+\frac{b^{n-1}}{s_{n} \alpha_{n}}\right\}=0 .
\end{aligned}
$$

Thus, applying Lemma 16 to (80), we immediately conclude that

$$
\lim _{n \rightarrow \infty} \frac{\left\|x_{n+1}-x_{n}\right\|}{\alpha_{n}}=0 .
$$

So, from $\lim _{n \rightarrow \infty} \alpha_{n}=0$ it follows that

$$
\lim _{n \rightarrow \infty}\left\|x_{n+1}-x_{n}\right\|=0
$$

Step 3. We prove that $\omega_{w}\left(x_{n}\right) \subset \Omega$ provided $\lim _{n \rightarrow \infty} \| x_{n}-$ $y_{n} \|=0$.

Indeed, first of all, let us show that $\| y_{n}-P_{C}(I-$ $(2 / L) \nabla f) y_{n}\|\rightarrow 0,\| x_{n}-u_{n}\|\rightarrow 0,\| x_{n}-v_{n} \| \rightarrow 0$, $\left\|v_{n}-G v_{n}\right\| \rightarrow 0$, and $\left\|v_{n}-W v_{n}\right\| \rightarrow 0$ as $n \rightarrow \infty$. As a matter of fact, utilizing Lemmas 7 and 15 we obtain from (54), (61), and (62) that

$$
\begin{aligned}
\| y_{n}- & p \|^{2} \\
= & \| \alpha_{n} \gamma\left(S v_{n}-S p\right)+\left(I-\alpha_{n} \mu F\right) W_{n} G v_{n} \\
& -\left(I-\alpha_{n} \mu F\right) p+\alpha_{n}(\gamma S-\mu F) p \|^{2} \\
\leq & \| \alpha_{n} \gamma\left(S v_{n}-S p\right) \\
& +\left(I-\alpha_{n} \mu F\right) W_{n} G v_{n}-\left(I-\alpha_{n} \mu F\right) p \|^{2} \\
& +2 \alpha_{n}\left\langle(\gamma S-\mu F) p, y_{n}-p\right\rangle \\
\leq & {\left[\alpha_{n} \gamma\left\|S v_{n}-S p\right\|\right.} \\
& \left.+\left\|\left(I-\alpha_{n} \mu F\right) W_{n} G v_{n}-\left(I-\alpha_{n} \mu F\right) p\right\|\right]^{2} \\
& +2 \alpha_{n}\left\langle(\gamma V-\mu F) p, y_{n}-p\right\rangle \\
\leq & {\left[\alpha_{n} \gamma\left\|v_{n}-p\right\|+\left(1-\alpha_{n} \tau\right)\left\|G v_{n}-p\right\|\right]^{2} } \\
& +2 \alpha_{n}\left\langle(\gamma S-\mu F) p, y_{n}-p\right\rangle \\
\leq & {\left[\alpha_{n} \tau\left\|v_{n}-p\right\|+\left(1-\alpha_{n} \tau\right)\left\|G v_{n}-p\right\|\right]^{2} } \\
& +2 \alpha_{n}\left\langle(\gamma S-\mu F) p, y_{n}-p\right\rangle \\
\leq & \alpha_{n} \tau\left\|v_{n}-p\right\|^{2}+\left(1-\alpha_{n} \tau\right)\left\|G v_{n}-p\right\|^{2}-p \|^{2}+2 \alpha_{n}\left\langle(\gamma S-\mu F) p, y_{n}-p\right\rangle . \\
& +2 \alpha_{n}\left\langle(\gamma S-\mu F) p, y_{n}-p\right\rangle \\
& +2 \alpha_{n} \tau\left\|v_{n}-p\right\|^{2}+\left(1-\alpha_{n} \tau\right)\left\|v_{n}-p\right\|^{2}\left\langle(\gamma S-\mu F) p, y_{n}-p\right\rangle \\
& \left\|v_{n}-p\right\|^{2}+2 \alpha_{n}\left\langle(\gamma S-\mu F) p, y_{n}-p\right\rangle \\
&
\end{aligned}
$$

Note that $x_{n+1}=s_{n} \gamma V x_{n}+\left(I-s_{n} \mu F\right) T_{n} y_{n}$. Hence we have

$$
x_{n+1}-y_{n}=s_{n}\left(\gamma V x_{n}-\mu F T_{n} y_{n}\right)+T_{n} y_{n}-y_{n},
$$

which yields

$$
\begin{aligned}
& \left\|T_{n} y_{n}-y_{n}\right\| \\
& \quad \leq\left\|x_{n+1}-y_{n}-s_{n}\left(\gamma V x_{n}-\mu F T_{n} y_{n}\right)\right\| \\
& \quad \leq\left\|x_{n+1}-y_{n}\right\|+s_{n}\left\|\gamma V x_{n}-\mu F T_{n} y_{n}\right\| \\
& \quad \leq\left\|x_{n+1}-x_{n}\right\|+\left\|x_{n}-y_{n}\right\|+s_{n}\left\|\gamma V x_{n}-\mu F T_{n} y_{n}\right\| .
\end{aligned}
$$

Since $\lim _{n \rightarrow \infty} s_{n}=0$ and $\lim _{n \rightarrow \infty}\left\|x_{n}-x_{n+1}\right\|=0$, from the assumption $\lim _{n \rightarrow \infty}\left\|x_{n}-y_{n}\right\|=0$ and the boundedness of $\left\{x_{n}\right\},\left\{y_{n}\right\}$, we obtain

$$
\lim _{n \rightarrow \infty}\left\|y_{n}-T_{n} y_{n}\right\|=0
$$


It is clear that

$$
\begin{aligned}
\left\|P_{C}\left(I-\theta_{n} \nabla f\right) y_{n}-y_{n}\right\| & =\left\|s_{n} y_{n}+\left(1-s_{n}\right) T_{n} y_{n}-y_{n}\right\| \\
& =\left(1-s_{n}\right)\left\|T_{n} y_{n}-y_{n}\right\| \\
& \leq\left\|T_{n} y_{n}-y_{n}\right\|,
\end{aligned}
$$

where $s_{n}=\left(\left(2-\theta_{n} L\right) / 4\right) \in(0,1 / 2)$ for each $\theta_{n} \in(0,2 / L)$. Hence we have

$$
\begin{aligned}
&\left\|P_{C}\left(I-\frac{2}{L} \nabla f\right) y_{n}-y_{n}\right\| \\
& \leq\left\|P_{C}\left(I-\frac{2}{L} \nabla f\right) y_{n}-P_{C}\left(I-\theta_{n} \nabla f\right) y_{n}\right\| \\
&+\left\|P_{C}\left(I-\theta_{n} \nabla f\right) y_{n}-y_{n}\right\| \\
& \leq\left\|\left(I-\frac{2}{L} \nabla f\right) y_{n}-\left(I-\theta_{n} \nabla f\right) y_{n}\right\| \\
&+\left\|P_{C}\left(I-\theta_{n} \nabla f\right) y_{n}-y_{n}\right\| \\
& \leq\left(\frac{2}{L}-\theta_{n}\right)\left\|\nabla f\left(y_{n}\right)\right\|+\left\|T_{n} y_{n}-y_{n}\right\| .
\end{aligned}
$$

From the boundedness of $\left\{y_{n}\right\}, s_{n} \rightarrow 0\left(\Leftrightarrow \theta_{n} \rightarrow 2 / L\right)$ and $\left\|T_{n} y_{n}-y_{n}\right\| \rightarrow 0$ (due to (87)), it follows that

$$
\lim _{n \rightarrow \infty}\left\|y_{n}-P_{C}\left(I-\frac{2}{L} \nabla f\right) y_{n}\right\|=0 .
$$

Also, from (30) it follows that for all $i \in\{1,2, \ldots, N\}$ and $k \in$ $\{1,2, \ldots, M\}$

$$
\begin{aligned}
\left\|v_{n}-p\right\|^{2}= & \left\|\Lambda_{n}^{N} u_{n}-p\right\|^{2} \\
\leq & \left\|\Lambda_{n}^{i} u_{n}-p\right\|^{2} \\
= & \left\|P_{C}\left(I-\lambda_{i, n} A_{i}\right) \Lambda_{n}^{i-1} u_{n}-P_{C}\left(I-\lambda_{i, n} A_{i}\right) p\right\|^{2} \\
\leq & \left\|\left(I-\lambda_{i, n} A_{i}\right) \Lambda_{n}^{i-1} u_{n}-\left(I-\lambda_{i, n} A_{i}\right) p\right\|^{2} \\
\leq & \left\|\Lambda_{n}^{i-1} u_{n}-p\right\|^{2} \\
& +\lambda_{i, n}\left(\lambda_{i, n}-2 \eta_{i}\right)\left\|A_{i} \Lambda_{n}^{i-1} u_{n}-A_{i} p\right\|^{2}
\end{aligned}
$$

$$
\begin{aligned}
& \leq\left\|u_{n}-p\right\|^{2}+\lambda_{i, n}\left(\lambda_{i, n}-2 \eta_{i}\right)\left\|A_{i} \Lambda_{n}^{i-1} u_{n}-A_{i} p\right\|^{2} \\
& \leq\left\|x_{n}-p\right\|^{2}+\lambda_{i, n}\left(\lambda_{i, n}-2 \eta_{i}\right)\left\|A_{i} \Lambda_{n}^{i-1} u_{n}-A_{i} p\right\|^{2} \text {, } \\
& \left\|u_{n}-p\right\|^{2}=\left\|\Delta_{n}^{M} x_{n}-p\right\|^{2} \\
& \leq\left\|\Delta_{n}^{k} x_{n}-p\right\|^{2} \\
& =\| T_{r_{k, n}}^{\left(\Theta_{k}, \varphi_{k}\right)}\left(I-r_{k, n} B_{k}\right) \Delta_{n}^{k-1} x_{n} \\
& -T_{r_{k, n}}^{\left(\Theta_{k}, \varphi_{k}\right)}\left(I-r_{k, n} B_{k}\right) p \|^{2} \\
& \leq\left\|\left(I-r_{k, n} B_{k}\right) \Delta_{n}^{k-1} x_{n}-\left(I-r_{k, n} B_{k}\right) p\right\|^{2} \\
& \leq\left\|\Delta_{n}^{k-1} x_{n}-p\right\|^{2} \\
& +r_{k, n}\left(r_{k, n}-2 \mu_{k}\right)\left\|B_{k} \Delta_{n}^{k-1} x_{n}-B_{k} p\right\|^{2} \\
& \leq\left\|x_{n}-p\right\|^{2} \\
& +r_{k, n}\left(r_{k, n}-2 \mu_{k}\right)\left\|B_{k} \Delta_{n}^{k-1} x_{n}-B_{k} p\right\|^{2} .
\end{aligned}
$$

So, from (84) and (91) it follows that

$$
\begin{aligned}
\| y_{n}- & p \|^{2} \\
\leq & \left\|v_{n}-p\right\|^{2}+2 \alpha_{n}\left\langle(\gamma S-\mu F) p, y_{n}-p\right\rangle \\
\leq & \left\|u_{n}-p\right\|^{2}+\lambda_{i, n}\left(\lambda_{i, n}-2 \eta_{i}\right)\left\|A_{i} \Lambda_{n}^{i-1} u_{n}-A_{i} p\right\|^{2} \\
& +2 \alpha_{n}\left\langle(\gamma S-\mu F) p, y_{n}-p\right\rangle \\
\leq & \left\|x_{n}-p\right\|^{2}+r_{k, n}\left(r_{k, n}-2 \mu_{k}\right)\left\|B_{k} \Delta_{n}^{k-1} x_{n}-B_{k} p\right\|^{2} \\
& +\lambda_{i, n}\left(\lambda_{i, n}-2 \eta_{i}\right)\left\|A_{i} \Lambda_{n}^{i-1} u_{n}-A_{i} p\right\|^{2} \\
& +2 \alpha_{n}\|(\gamma S-\mu F) p\|\left\|y_{n}-p\right\|,
\end{aligned}
$$

which hence leads to

$$
\begin{aligned}
& r_{k, n}\left(2 \mu_{k}-r_{k, n}\right)\left\|B_{k} \Delta_{n}^{k-1} x_{n}-B_{k} p\right\|^{2} \\
&+\lambda_{i, n}\left(2 \eta_{i}-\lambda_{i, n}\right)\left\|A_{i} \Lambda_{n}^{i-1} u_{n}-A_{i} p\right\|^{2} \\
& \leq\left\|x_{n}-p\right\|^{2}-\left\|y_{n}-p\right\|^{2} \\
&+2 \alpha_{n}\|(\gamma S-\mu F) p\|\left\|y_{n}-p\right\| \\
& \leq\left\|x_{n}-y_{n}\right\|\left(\left\|x_{n}-p\right\|+\left\|y_{n}-p\right\|\right) \\
&+2 \alpha_{n}\|(\gamma S-\mu F) p\|\left\|y_{n}-p\right\| .
\end{aligned}
$$

Since $\lim _{n \rightarrow \infty} \alpha_{n}=0,\left\{\lambda_{i, n}\right\} \subset\left[a_{i}, b_{i}\right] \subset\left(0,2 \eta_{i}\right)$ and $\left\{r_{k, n}\right\} \subset\left[e_{k}, f_{k}\right] \subset\left(0,2 \mu_{k}\right)$ for all $i \in\{1,2, \ldots, N\}$ and $k \in\{1,2, \ldots, M\}$, by the assumption $\lim _{n \rightarrow \infty}\left\|x_{n}-y_{n}\right\|=0$ 
16

Journal of Applied Mathematics

and the boundedness of $\left\{x_{n}\right\},\left\{y_{n}\right\}$, we conclude immediately that

$$
\begin{aligned}
& \lim _{n \rightarrow \infty}\left\|A_{i} \Lambda_{n}^{i-1} u_{n}-A_{i} p\right\|=0, \\
& \lim _{n \rightarrow \infty}\left\|B_{k} \Delta_{n}^{k-1} x_{n}-B_{k} p\right\|=0,
\end{aligned}
$$

for all $i \in\{1,2, \ldots, N\}$ and $k \in\{1,2, \ldots, M\}$.

Furthermore, by Proposition 6(ii) we obtain that for each $k \in\{1,2, \ldots, M\}$

$$
\begin{aligned}
& \left\|\Delta_{n}^{k} x_{n}-p\right\|^{2} \\
& =\left\|T_{r_{k, n}}^{\left(\Theta_{k}, \varphi_{k}\right)}\left(I-r_{k, n} B_{k}\right) \Delta_{n}^{k-1} x_{n}-T_{r_{k, n}}^{\left(\Theta_{k}, \varphi_{k}\right)}\left(I-r_{k, n} B_{k}\right) p\right\|^{2} \\
& \leq\left\langle\left(I-r_{k, n} B_{k}\right) \Delta_{n}^{k-1} x_{n}-\left(I-r_{k, n} B_{k}\right) p, \Delta_{n}^{k} x_{n}-p\right\rangle \\
& =\frac{1}{2}\left(\left\|\left(I-r_{k, n} B_{k}\right) \Delta_{n}^{k-1} x_{n}-\left(I-r_{k, n} B_{k}\right) p\right\|^{2}\right. \\
& \quad+\left\|\Delta_{n}^{k} x_{n}-p\right\|^{2}-\|\left(I-r_{k, n} B_{k}\right) \Delta_{n}^{k-1} x_{n} \\
& \left.\quad-\left(I-r_{k, n} B_{k}\right) p-\left(\Delta_{n}^{k} x_{n}-p\right) \|^{2}\right) \\
& \leq \frac{1}{2}\left(\left\|\Delta_{n}^{k-1} x_{n}-p\right\|^{2}+\left\|\Delta_{n}^{k} x_{n}-p\right\|^{2}\right. \\
& \left.\quad-\left\|\Delta_{n}^{k-1} x_{n}-\Delta_{n}^{k} x_{n}-r_{k, n}\left(B_{k} \Delta_{n}^{k-1} x_{n}-B_{k} p\right)\right\|^{2}\right),
\end{aligned}
$$

$$
\begin{aligned}
\leq & \left\|x_{n}-p\right\|^{2}-\left\|\Delta_{n}^{k-1} x_{n}-\Delta_{n}^{k} x_{n}\right\|^{2} \\
& +2 r_{k, n}\left\|\Delta_{n}^{k-1} x_{n}-\Delta_{n}^{k} x_{n}\right\|\left\|B_{k} \Delta_{n}^{k-1} x_{n}-B_{k} p\right\| .
\end{aligned}
$$

Also, by Proposition 1(iii), we obtain that for each $i \in$ $\{1,2, \ldots, N\}$

$$
\begin{gathered}
\left\|\Lambda_{n}^{i} u_{n}-p\right\|^{2} \\
=\left\|P_{C}\left(I-\lambda_{i, n} A_{i}\right) \Lambda_{n}^{i-1} u_{n}-P_{C}\left(I-\lambda_{i, n} A_{i}\right) p\right\|^{2} \\
\leq\left\langle\left(I-\lambda_{i, n} A_{i}\right) \Lambda_{n}^{i-1} u_{n}-\left(I-\lambda_{i, n} A_{i}\right) p, \Lambda_{n}^{i} u_{n}-p\right\rangle \\
=\frac{1}{2}\left(\left\|\left(I-\lambda_{i, n} A_{i}\right) \Lambda_{n}^{i-1} u_{n}-\left(I-\lambda_{i, n} A_{i}\right) p\right\|^{2}\right. \\
\quad+\left\|\Lambda_{n}^{i} u_{n}-p\right\|^{2} \\
\quad-\|\left(I-\lambda_{i, n} A_{i}\right) \Lambda_{n}^{i-1} u_{n}-\left(I-\lambda_{i, n} A_{i}\right) p \\
\left.\quad-\left(\Lambda_{n}^{i} u_{n}-p\right) \|^{2}\right) \\
\leq \frac{1}{2}\left(\left\|\Lambda_{n}^{i-1} u_{n}-p\right\|^{2}+\left\|\Lambda_{n}^{i} u_{n}-p\right\|^{2}\right. \\
\left.\quad-\left\|\Lambda_{n}^{i-1} u_{n}-\Lambda_{n}^{i} u_{n}-\lambda_{i, n}\left(A_{i} \Lambda_{n}^{i-1} u_{n}-A_{i} p\right)\right\|^{2}\right) \\
\leq \frac{1}{2}\left(\left\|u_{n}-p\right\|^{2}+\left\|\Lambda_{n}^{i} u_{n}-p\right\|^{2}\right. \\
\left.\quad\left\|\Lambda_{n}^{i-1} u_{n}-\Lambda_{n}^{i} u_{n}-\lambda_{i, n}\left(A_{i} \Lambda_{n}^{i-1} u_{n}-A_{i} p\right)\right\|^{2}\right)
\end{gathered}
$$

which implies that

which implies

$$
\begin{aligned}
&\left\|\Delta_{n}^{k} x_{n}-p\right\|^{2} \\
& \leq\left\|\Delta_{n}^{k-1} x_{n}-p\right\|^{2} \\
& \quad-\left\|\Delta_{n}^{k-1} x_{n}-\Delta_{n}^{k} x_{n}-r_{k, n}\left(B_{k} \Delta_{n}^{k-1} x_{n}-B_{k} p\right)\right\|^{2} \\
&=\left\|\Delta_{n}^{k-1} x_{n}-p\right\|^{2}-\left\|\Delta_{n}^{k-1} x_{n}-\Delta_{n}^{k} x_{n}\right\|^{2} \\
& \quad-r_{k, n}^{2}\left\|B_{k} \Delta_{n}^{k-1} x_{n}-B_{k} \mathrm{p}\right\|^{2} \\
& \quad+2 r_{k, n}\left\langle\Delta_{n}^{k-1} x_{n}-\Delta_{n}^{k} x_{n}, B_{k} \Delta_{n}^{k-1} x_{n}-B_{k} p\right\rangle \\
& \leq\left\|\Delta_{n}^{k-1} x_{n}-p\right\|^{2}-\left\|\Delta_{n}^{k-1} x_{n}-\Delta_{n}^{k} x_{n}\right\|^{2} \\
&+2 r_{k, n}\left\|\Delta_{n}^{k-1} x_{n}-\Delta_{n}^{k} x_{n}\right\|\left\|B_{k} \Delta_{n}^{k-1} x_{n}-B_{k} p\right\|
\end{aligned}
$$

$$
\begin{aligned}
\| \Lambda_{n}^{i} u_{n}- & p \|^{2} \\
\leq & \left\|u_{n}-p\right\|^{2} \\
& \quad-\left\|\Lambda_{n}^{i-1} u_{n}-\Lambda_{n}^{i} u_{n}-\lambda_{i, n}\left(A_{i} \Lambda_{n}^{i-1} u_{n}-A_{i} p\right)\right\|^{2} \\
= & \left\|u_{n}-p\right\|^{2} \\
& -\left\|\Lambda_{n}^{i-1} u_{n}-\Lambda_{n}^{i} u_{n}\right\|^{2}-\lambda_{i, n}^{2}\left\|A_{i} \Lambda_{n}^{i-1} u_{n}-A_{i} p\right\|^{2} \\
& +2 \lambda_{i, n}\left\langle\Lambda_{n}^{i-1} u_{n}-\Lambda_{n}^{i} u_{n}, A_{i} \Lambda_{n}^{i-1} u_{n}-A_{i} p\right\rangle \\
\leq & \left\|u_{n}-p\right\|^{2}-\left\|\Lambda_{n}^{i-1} u_{n}-\Lambda_{n}^{i} u_{n}\right\|^{2} \\
& +2 \lambda_{i, n}\left\|\Lambda_{n}^{i-1} u_{n}-\Lambda_{n}^{i} u_{n}\right\|\left\|A_{i} \Lambda_{n}^{i-1} u_{n}-A_{i} p\right\| .
\end{aligned}
$$


Thus, from (84), (96), and (98), we have

$$
\begin{aligned}
\| y_{n}- & p \|^{2} \\
\leq & \left\|v_{n}-p\right\|^{2}+2 \alpha_{n}\left\langle(\gamma S-\mu F) p, y_{n}-p\right\rangle \\
\leq & \left\|\Lambda_{n}^{i} u_{n}-p\right\|^{2}+2 \alpha_{n}\left\langle(\gamma S-\mu \mathrm{F}) p, y_{n}-p\right\rangle \\
\leq & \left\|u_{n}-p\right\|^{2}-\left\|\Lambda_{n}^{i-1} u_{n}-\Lambda_{n}^{i} u_{n}\right\|^{2} \\
& +2 \lambda_{i, n}\left\|\Lambda_{n}^{i-1} u_{n}-\Lambda_{n}^{i} u_{n}\right\|\left\|A_{i} \Lambda_{n}^{i-1} u_{n}-A_{i} p\right\| \\
& +2 \alpha_{n}\left\langle(\gamma S-\mu F) p, y_{n}-p\right\rangle \\
\leq & \left\|\Delta_{n}^{k} x_{n}-p\right\|^{2}-\left\|\Lambda_{n}^{i-1} u_{n}-\Lambda_{n}^{i} u_{n}\right\|^{2} \\
& +2 \lambda_{i, n}\left\|\Lambda_{n}^{i-1} u_{n}-\Lambda_{n}^{i} u_{n}\right\|\left\|A_{i} \Lambda_{n}^{i-1} u_{n}-A_{i} p\right\| \\
& +2 \alpha_{n}\left\langle(\gamma S-\mu F) p, y_{n}-p\right\rangle \\
\leq & \left\|x_{n}-p\right\|^{2}-\left\|\Delta_{n}^{k-1} x_{n}-\Delta_{n}^{k} x_{n}\right\|^{2} \\
& +2 r_{k, n}\left\|\Delta_{n}^{k-1} x_{n}-\Delta_{n}^{k} x_{n}\right\|\left\|B_{k} \Delta_{n}^{k-1} x_{n}-B_{k} p\right\| \\
& -\left\|\Lambda_{n}^{i-1} u_{n}-\Lambda_{n}^{i} u_{n}\right\|^{2} \\
& +2 \lambda_{i, n}\left\|\Lambda_{n}^{i-1} u_{n}-\Lambda_{n}^{i} u_{n}\right\|\left\|A_{i} \Lambda_{n}^{i-1} u_{n}-A_{i} p\right\| \\
& +2 \alpha_{n}\|(\gamma S-\mu F) p\|\left\|y_{n}-p\right\|,
\end{aligned}
$$

which yields

$$
\begin{aligned}
&\left\|\Delta_{n}^{k-1} x_{n}-\Delta_{n}^{k} x_{n}\right\|^{2}+\left\|\Lambda_{n}^{i-1} u_{n}-\Lambda_{n}^{i} u_{n}\right\|^{2} \\
& \leq\left\|x_{n}-p\right\|^{2}-\left\|y_{n}-p\right\|^{2} \\
&+2 r_{k, n}\left\|\Delta_{n}^{k-1} x_{n}-\Delta_{n}^{k} x_{n}\right\|\left\|B_{k} \Delta_{n}^{k-1} x_{n}-B_{k} p\right\| \\
&+2 \lambda_{i, n}\left\|\Lambda_{n}^{i-1} u_{n}-\Lambda_{n}^{i} u_{n}\right\|\left\|A_{i} \Lambda_{n}^{i-1} u_{n}-A_{i} p\right\| \\
&+2 \alpha_{n}\|(\gamma S-\mu F) p\|\left\|y_{n}-p\right\| \\
& \leq\left\|x_{n}-y_{n}\right\|\left(\left\|x_{n}-p\right\|+\left\|y_{n}-p\right\|\right) \\
&+2 r_{k, n}\left\|\Delta_{n}^{k-1} x_{n}-\Delta_{n}^{k} x_{n}\right\|\left\|B_{k} \Delta_{n}^{k-1} x_{n}-B_{k} p\right\| \\
&+2 \lambda_{i, n}\left\|\Lambda_{n}^{i-1} u_{n}-\Lambda_{n}^{i} u_{n}\right\|\left\|A_{i} \Lambda_{n}^{i-1} u_{n}-A_{i} p\right\| \\
&+2 \alpha_{n}\|(\gamma S-\mu F) p\|\left\|y_{n}-p\right\| .
\end{aligned}
$$

Since $\lim _{n \rightarrow \infty} \alpha_{n}=0,\left\{x_{n}\right\},\left\{y_{n}\right\}$, and $\left\{u_{n}\right\}$ are bounded. For all $i \in\{1,2, \ldots, N\}$ and $k \in\{1,2, \ldots, M\}$, we have $\left\{\lambda_{i, n}\right\} \subset$ $\left[a_{i}, b_{i}\right] \subset\left(0,2 \eta_{i}\right)$ and $\left\{r_{k, n}\right\} \subset\left[e_{k}, f_{k}\right] \subset\left(0,2 \mu_{k}\right)$, then by (94) and the assumption $\lim _{n \rightarrow \infty}\left\|x_{n}-y_{n}\right\|=0$, we conclude immediately that

$$
\begin{gathered}
\lim _{n \rightarrow \infty}\left\|\Lambda_{n}^{i-1} u_{n}-\Lambda_{n}^{i} u_{n}\right\|, \\
\lim _{n \rightarrow \infty}\left\|\Delta_{n}^{k-1} x_{n}-\Delta_{n}^{k} x_{n}\right\|=0,
\end{gathered}
$$

for all $i \in\{1,2, \ldots, N\}$ and $k \in\{1,2, \ldots, M\}$. Note that

$$
\begin{aligned}
\left\|x_{n}-u_{n}\right\|= & \left\|\Delta_{n}^{0} x_{n}-\Delta_{n}^{M} x_{n}\right\| \\
\leq & \left\|\Delta_{n}^{0} x_{n}-\Delta_{n}^{1} x_{n}\right\| \\
& +\left\|\Delta_{n}^{1} x_{n}-\Delta_{n}^{2} x_{n}\right\|+\cdots \\
& +\left\|\Delta_{n}^{M-1} x_{n}-\Delta_{n}^{M} x_{n}\right\|, \\
\left\|u_{n}-v_{n}\right\|= & \left\|\Lambda_{n}^{0} u_{n}-\Lambda_{n}^{N} u_{n}\right\| \\
\leq & \left\|\Lambda_{n}^{0} u_{n}-\Lambda_{n}^{1} u_{n}\right\| \\
& +\left\|\Lambda_{n}^{1} u_{n}-\Lambda_{n}^{2} u_{n}\right\|+\cdots \\
& +\left\|\Lambda_{n}^{N-1} u_{n}-\Lambda_{n}^{N} u_{n}\right\| .
\end{aligned}
$$

Thus, from (101) we have

$$
\lim _{n \rightarrow \infty}\left\|x_{n}-u_{n}\right\|=0, \quad \lim _{n \rightarrow \infty}\left\|u_{n}-v_{n}\right\|=0 .
$$

It is easy to see that as $n \rightarrow \infty$

$$
\left\|x_{n}-v_{n}\right\| \leq\left\|x_{n}-u_{n}\right\|+\left\|u_{n}-v_{n}\right\| \longrightarrow 0 .
$$

On the other hand, for simplicity, we write $\widetilde{p}=P_{C}(I-$ $\left.v_{2} F_{2}\right) p, \widetilde{v}_{n}=P_{C}\left(I-v_{2} F_{2}\right) v_{n}$, and $w_{n}=G v_{n}=P_{C}\left(I-v_{1} F_{1}\right) \widetilde{v}_{n}$ for all $n \geq 1$. Then

$$
\begin{aligned}
p & =G p \\
& =P_{C}\left(I-v_{1} F_{1}\right) \tilde{p} \\
& =P_{C}\left(I-v_{1} F_{1}\right) P_{C}\left(I-v_{2} F_{2}\right) p .
\end{aligned}
$$

We now show that $\lim _{n \rightarrow \infty}\left\|G v_{n}-v_{n}\right\|=0$; that is, $\lim _{n \rightarrow \infty}\left\|w_{n}-v_{n}\right\|=0$. As a matter of fact, for $p \in \Omega$, it follows from (61), (62), and (84) that

$$
\begin{aligned}
& \left\|y_{n}-p\right\|^{2} \\
& \quad \leq \alpha_{n} \tau\left\|v_{n}-p\right\|^{2}+\left(1-\alpha_{n} \tau\right)\left\|G v_{n}-p\right\|^{2} \\
& \quad+2 \alpha_{n}\left\langle(\gamma S-\mu F) p, y_{n}-p\right\rangle
\end{aligned}
$$




$$
\begin{aligned}
\leq & \alpha_{n} \tau\left\|v_{n}-p\right\|^{2}+\left(1-\alpha_{n} \tau\right)\left\|w_{n}-p\right\|^{2} \\
& +2 \alpha_{n}\|(\gamma S-\mu F) p\|\left\|y_{n}-p\right\| \\
\leq & \alpha_{n} \tau\left\|v_{n}-p\right\|^{2}+\left(1-\alpha_{n} \tau\right) \\
& \times\left[\left\|\widetilde{v}_{n}-\tilde{p}\right\|^{2}+v_{1}\left(v_{1}-2 \zeta_{1}\right)\left\|F_{1} \widetilde{v}_{n}-F_{1} \tilde{p}\right\|^{2}\right] \\
& +2 \alpha_{n}\|(\gamma S-\mu F) p\|\left\|y_{n}-p\right\| \\
\leq & \alpha_{n} \tau\left\|v_{n}-p\right\|^{2}+\left(1-\alpha_{n} \tau\right) \\
& \times\left[\left\|v_{n}-p\right\|^{2}+v_{2}\left(v_{2}-2 \zeta_{2}\right)\left\|F_{2} v_{n}-F_{2} p\right\|^{2}\right. \\
& \left.+v_{1}\left(v_{1}-2 \zeta_{1}\right)\left\|F_{1} \widetilde{v}_{n}-F_{1} \tilde{p}\right\|^{2}\right] \\
& +2 \alpha_{n}\|(\gamma S-\mu F) p\|\left\|y_{n}-p\right\| \\
= & \left\|v_{n}-p\right\|^{2}+\left(1-\alpha_{n} \tau\right) \\
& \times\left[v_{2}\left(v_{2}-2 \zeta_{2}\right)\left\|F_{2} v_{n}-F_{2} p\right\|^{2}\right. \\
& \left.+v_{1}\left(v_{1}-2 \zeta_{1}\right)\left\|F_{1} \widetilde{v}_{n}-F_{1} \tilde{p}\right\|^{2}\right] \\
& +2 \alpha_{n}\|(\gamma S-\mu F) p\|\left\|y_{n}-p\right\| \\
\leq & \left\|x_{n}-p\right\|^{2}+\left(1-\alpha_{n} \tau\right) \\
& \times\left[v_{2}\left(v_{2}-2 \zeta_{2}\right)\left\|F_{2} v_{n}-F_{2} p\right\|^{2}\right. \\
& \left.+v_{1}\left(v_{1}-2 \zeta_{1}\right)\left\|F_{1} \widetilde{v}_{n}-F_{1} \tilde{p}\right\|^{2}\right] \\
+ & 2 \alpha_{n}\|(\gamma S-\mu F) p\|\left\|y_{n}-p\right\|,
\end{aligned}
$$

which immediately yields

$$
\begin{gathered}
\left(1-\alpha_{n} \tau\right)\left[v_{2}\left(2 \zeta_{2}-v_{2}\right)\left\|F_{2} v_{n}-F_{2} p\right\|^{2}\right. \\
\left.+v_{1}\left(2 \zeta_{1}-v_{1}\right)\left\|F_{1} \widetilde{v}_{n}-F_{1} \tilde{p}\right\|^{2}\right] \\
\leq\left\|x_{n}-p\right\|^{2}-\left\|y_{n}-p\right\|^{2} \\
+2 \alpha_{n}\|(\gamma S-\mu F) p\|\left\|y_{n}-p\right\| \\
\leq\left\|x_{n}-y_{n}\right\|\left(\left\|x_{n}-p\right\|+\left\|y_{n}-p\right\|\right) \\
+2 \alpha_{n}\|(\gamma S-\mu F) p\|\left\|y_{n}-p\right\| .
\end{gathered}
$$

Since $\lim _{n \rightarrow \infty} \alpha_{n}=0, v_{j} \in\left(0,2 \zeta_{j}\right)$ for $j=1,2$ and $\left\{x_{n}\right\}$ and $\left\{y_{n}\right\}$ are bounded, by the assumption $\lim _{n \rightarrow \infty}\left\|x_{n}-y_{n}\right\|=0$, we get

$$
\lim _{n \rightarrow \infty}\left\|F_{2} v_{n}-F_{2} p\right\|=0, \quad \lim _{n \rightarrow \infty}\left\|F_{1} \widetilde{v}_{n}-F_{1} \tilde{p}\right\|=0 .
$$

Also, in terms of the firm nonexpansivity of $P_{C}$ and the $\zeta_{j}$ inverse-strong monotonicity of $F_{j}$ for $j=1,2$, we obtain from $v_{j} \in\left(0,2 \zeta_{j}\right), j=1,2$ and (67) that

$$
\begin{aligned}
& \left\|\widetilde{v}_{n}-\tilde{p}\right\|^{2} \\
& =\left\|P_{C}\left(I-v_{2} F_{2}\right) v_{n}-P_{C}\left(I-v_{2} F_{2}\right) p\right\|^{2} \\
& \leq\left\langle\left(I-v_{2} F_{2}\right) v_{n}-\left(I-v_{2} F_{2}\right) p, \widetilde{v}_{n}-\tilde{p}\right\rangle \\
& =\frac{1}{2}\left[\left\|\left(I-v_{2} F_{2}\right) v_{n}-\left(I-v_{2} F_{2}\right) p\right\|^{2}+\left\|\widetilde{v}_{n}-\tilde{p}\right\|^{2}\right. \\
& \left.-\left\|\left(I-v_{2} F_{2}\right) v_{n}-\left(I-v_{2} F_{2}\right) p-\left(\widetilde{v}_{n}-\tilde{p}\right)\right\|^{2}\right] \\
& \leq \frac{1}{2}\left[\left\|v_{n}-p\right\|^{2}+\left\|\widetilde{v}_{n}-\tilde{p}\right\|^{2}\right. \\
& \left.-\left\|\left(v_{n}-\widetilde{v}_{n}\right)-v_{2}\left(F_{2} v_{n}-F_{2} p\right)-(p-\tilde{p})\right\|^{2}\right] \\
& =\frac{1}{2}\left[\left\|v_{n}-p\right\|^{2}+\left\|\widetilde{v}_{n}-\tilde{p}\right\|^{2}\right. \\
& -\left\|\left(v_{n}-\widetilde{v}_{n}\right)-(p-\widetilde{p})\right\|^{2} \\
& +2 v_{2}\left\langle\left(v_{n}-\widetilde{v}_{n}\right)-(p-\widetilde{p}), F_{2} v_{n}-F_{2} p\right\rangle \\
& \left.-v_{2}^{2}\left\|F_{2} v_{n}-F_{2} p\right\|^{2}\right] \text {, } \\
& \left\|w_{n}-p\right\|^{2} \\
& =\left\|P_{C}\left(I-v_{1} F_{1}\right) \widetilde{v}_{n}-P_{C}\left(I-v_{1} F_{1}\right) \tilde{p}\right\|^{2} \\
& \leq\left\langle\left(I-v_{1} F_{1}\right) \widetilde{v}_{n}-\left(I-v_{1} F_{1}\right) \tilde{p}, w_{n}-p\right\rangle \\
& =\frac{1}{2}\left[\left\|\left(I-v_{1} F_{1}\right) \widetilde{v}_{n}-\left(I-v_{1} F_{1}\right) \tilde{p}\right\|^{2}+\left\|w_{n}-p\right\|^{2}\right. \\
& \left.-\left\|\left(I-\nu_{1} F_{1}\right) \widetilde{v}_{n}-\left(I-\nu_{1} F_{1}\right) \tilde{p}-\left(w_{n}-p\right)\right\|^{2}\right] \\
& \leq \frac{1}{2}\left[\left\|\widetilde{v}_{n}-\widetilde{p}\right\|^{2}+\left\|w_{n}-p\right\|^{2}\right. \\
& -\left\|\left(\widetilde{v}_{n}-w_{n}\right)+(p-\tilde{p})\right\|^{2} \\
& +2 v_{1}\left\langle F_{1} \widetilde{v}_{n}-F_{1} \tilde{p},\left(\widetilde{v}_{n}-w_{n}\right)+(p-\widetilde{p})\right\rangle \\
& \left.-v_{1}^{2}\left\|F_{1} \widetilde{v}_{n}-F_{1} \tilde{p}\right\|^{2}\right] \\
& \leq \frac{1}{2}\left[\left\|v_{n}-p\right\|^{2}+\left\|w_{n}-p\right\|^{2}\right. \\
& -\left\|\left(\widetilde{v}_{n}-w_{n}\right)+(p-\tilde{p})\right\|^{2} \\
& \left.+2 v_{1}\left\langle F_{1} \widetilde{v}_{n}-F_{1} \tilde{p},\left(\widetilde{v}_{n}-w_{n}\right)+(p-\tilde{p})\right\rangle\right] .
\end{aligned}
$$

Thus, we have

$$
\begin{aligned}
\left\|\widetilde{v}_{n}-\tilde{p}\right\|^{2} \leq & \left\|v_{n}-p\right\|^{2} \\
& -\left\|\left(v_{n}-\widetilde{v}_{n}\right)-(p-\widetilde{p})\right\|^{2}
\end{aligned}
$$




$$
\begin{aligned}
& +2 v_{2}\left\langle\left(v_{n}-\widetilde{v}_{n}\right)-(p-\tilde{p}), F_{2} v_{n}-F_{2} p\right\rangle \\
& -v_{2}^{2}\left\|F_{2} v_{n}-F_{2} p\right\|^{2},
\end{aligned}
$$

$$
\begin{aligned}
\left\|w_{n}-p\right\|^{2} \leq & \left\|v_{n}-p\right\|^{2} \\
& -\left\|\left(\widetilde{v}_{n}-w_{n}\right)+(p-\widetilde{p})\right\|^{2} \\
& +2 v_{1}\left\|F_{1} \widetilde{v}_{n}-F_{1} \tilde{p}\right\|\left\|\left(\widetilde{v}_{n}-w_{n}\right)+(p-\tilde{p})\right\| .
\end{aligned}
$$

Consequently, from (61), (106), and (110) it follows that

$$
\begin{aligned}
\| y_{n}- & p \|^{2} \\
\leq & \alpha_{n} \tau\left\|v_{n}-p\right\|^{2}+\left(1-\alpha_{n} \tau\right) \\
& \times\left[\left\|\widetilde{v}_{n}-\tilde{p}\right\|^{2}+v_{1}\left(v_{1}-2 \zeta_{1}\right)\left\|F_{1} \widetilde{v}_{n}-F_{1} \tilde{p}\right\|^{2}\right] \\
& +2 \alpha_{n}\|(\gamma S-\mu F) p\|\left\|y_{n}-p\right\| \\
\leq & \alpha_{n} \tau\left\|v_{n}-p\right\|^{2}+\left(1-\alpha_{n} \tau\right)\left\|\widetilde{v}_{n}-\tilde{p}\right\|^{2} \\
& +2 \alpha_{n}\|(\gamma S-\mu F) p\|\left\|y_{n}-p\right\| \\
\leq & \alpha_{n} \tau\left\|v_{n}-p\right\|^{2}+\left(1-\alpha_{n} \tau\right) \\
& \times\left[\left\|v_{n}-p\right\|^{2}-\left\|\left(v_{n}-\widetilde{v}_{n}\right)-(p-\widetilde{p})\right\|^{2}\right. \\
& +2 v_{2}\left\langle\left(v_{n}-\widetilde{v}_{n}\right)-(p-\widetilde{p}), F_{2} v_{n}-F_{2} p\right\rangle \\
& \left.-v_{2}^{2}\left\|F_{2} v_{n}-F_{2} p\right\|^{2}\right] \\
+ & 2 \alpha_{n}\|(\gamma S-\mu F) p\|\left\|y_{n}-p\right\| \\
\leq & \alpha_{n} \tau\left\|v_{n}-p\right\|^{2}+\left(1-\alpha_{n} \tau\right) \\
& \times\left[\left\|v_{n}-p\right\|^{2}-\left\|\left(v_{n}-\widetilde{v}_{n}\right)-(p-\widetilde{p})\right\|^{2}\right. \\
& \left.+2 v_{2}\left\|\left(v_{n}-\widetilde{v}_{n}\right)-(p-\widetilde{p})\right\|\left\|F_{2} v_{n}-F_{2} p\right\|\right] \\
+ & 2 \alpha_{n}\|(\gamma S-\mu F) p\|\left\|y_{n}-p\right\| \\
\leq & \left\|v_{n}-p\right\|^{2}-\left(1-\alpha_{n} \tau\right)\left\|\left(v_{n}-\widetilde{v}_{n}\right)-(p-\widetilde{p})\right\|^{2} \\
+ & 2 v_{2}\left\|\left(v_{n}-\widetilde{v}_{n}\right)-(p-\widetilde{p})\right\|\left\|F_{2} v_{n}-F_{2} p\right\| \\
+ & 2 \alpha_{n}\|(\gamma S-\mu F) p\|\left\|y_{n}-p\right\| \\
& +2 v_{2}\left\|\left(v_{n}-\widetilde{v}_{n}\right)-(p-\widetilde{p})\right\|\left\|F_{2} v_{n}-F_{2} p\right\| \\
& 2 \alpha_{n}\|(\gamma S-\mu F) p\|\left\|y_{n}-p\right\|, \\
&
\end{aligned}
$$

which hence leads to

$$
\begin{gathered}
\left(1-\alpha_{n} \tau\right)\left\|\left(v_{n}-\widetilde{v}_{n}\right)-(p-\tilde{p})\right\|^{2} \\
\leq\left\|x_{n}-p\right\|^{2}-\left\|y_{n}-p\right\|^{2}
\end{gathered}
$$

$$
\begin{aligned}
& +2 v_{2}\left\|\left(v_{n}-\widetilde{v}_{n}\right)-(p-\widetilde{p})\right\|\left\|F_{2} v_{n}-F_{2} p\right\| \\
& +2 \alpha_{n}\|(\gamma S-\mu F) p\|\left\|y_{n}-p\right\| \\
\leq & \left\|x_{n}-y_{n}\right\|\left(\left\|x_{n}-p\right\|+\left\|y_{n}-p\right\|\right) \\
& +2 v_{2}\left\|\left(v_{n}-\widetilde{v}_{n}\right)-(p-\widetilde{p})\right\|\left\|F_{2} v_{n}-F_{2} p\right\| \\
& +2 \alpha_{n}\|(\gamma S-\mu F) p\|\left\|y_{n}-p\right\| .
\end{aligned}
$$

Since $\lim _{n \rightarrow \infty} \alpha_{n}=0,\left\{x_{n}\right\},\left\{y_{n}\right\},\left\{v_{n}\right\}$, and $\left\{\widetilde{v}_{n}\right\}$ are bounded sequences, by the assumption $\lim _{n \rightarrow \infty}\left\|x_{n}-y_{n}\right\|=0$, we conclude from (108) that

$$
\lim _{n \rightarrow \infty}\left\|\left(v_{n}-\widetilde{v}_{n}\right)-(p-\widetilde{p})\right\|=0 .
$$

Furthermore, from (62), (106), and (111) it follows that

$$
\begin{aligned}
\| y_{n}- & p \|^{2} \\
\leq & \alpha_{n} \tau\left\|v_{n}-p\right\|^{2}+\left(1-\alpha_{n} \tau\right)\left\|w_{n}-p\right\|^{2} \\
& +2 \alpha_{n}\|(\gamma S-\mu F) p\|\left\|y_{n}-p\right\| \\
\leq & \alpha_{n} \tau\left\|v_{n}-p\right\|^{2}+\left(1-\alpha_{n} \tau\right) \\
& \times\left[\left\|v_{n}-p\right\|^{2}-\left\|\left(\widetilde{v}_{n}-w_{n}\right)+(p-\tilde{p})\right\|^{2}\right. \\
& \left.\quad+2 v_{1}\left\|F_{1} \widetilde{v}_{n}-F_{1} \tilde{p}\right\|\left\|\left(\widetilde{v}_{n}-w_{n}\right)+(p-\widetilde{p})\right\|\right] \\
& +2 \alpha_{n}\|(\gamma S-\mu F) p\|\left\|y_{n}-p\right\| \\
\leq & \left\|v_{n}-p\right\|^{2}-\left(1-\alpha_{n} \tau\right)\left\|\left(\widetilde{v}_{n}-w_{n}\right)+(p-\tilde{p})\right\|^{2} \\
& +2 v_{1}\left\|F_{1} \widetilde{v}_{n}-F_{1} \tilde{p}\right\|\left\|\left(\widetilde{v}_{n}-w_{n}\right)+(p-\tilde{p})\right\| \\
& +2 \alpha_{n}\|(\gamma S-\mu F) p\|\left\|y_{n}-p\right\| \\
\leq & \left\|x_{n}-p\right\|^{2}-\left(1-\alpha_{n} \tau\right)\left\|\left(\widetilde{v}_{n}-w_{n}\right)+(p-\widetilde{p})\right\|^{2} \\
& +2 v_{1}\left\|F_{1} \widetilde{v}_{n}-F_{1} \tilde{p}\right\|\left\|\left(\widetilde{v}_{n}-w_{n}\right)+(p-\widetilde{p})\right\| \\
& +2 \alpha_{n}\|(\gamma S-\mu F) p\|\left\|y_{n}-p\right\|,
\end{aligned}
$$

which hence yields

$$
\begin{aligned}
\left(1-\alpha_{n} \tau\right) & \left\|\left(\widetilde{v}_{n}-w_{n}\right)+(p-\tilde{p})\right\|^{2} \\
\leq & \left\|x_{n}-p\right\|^{2}-\left\|y_{n}-p\right\|^{2} \\
& +2 v_{1}\left\|F_{1} \widetilde{v}_{n}-F_{1} \tilde{p}\right\|\left\|\left(\widetilde{v}_{n}-w_{n}\right)+(p-\widetilde{p})\right\| \\
& +2 \alpha_{n}\|(\gamma S-\mu F) p\|\left\|y_{n}-p\right\| \\
\leq & \left\|x_{n}-y_{n}\right\|\left(\left\|x_{n}-p\right\|+\left\|y_{n}-p\right\|\right) \\
& +2 v_{1}\left\|F_{1} \widetilde{v}_{n}-F_{1} \tilde{p}\right\|\left\|\left(\widetilde{v}_{n}-w_{n}\right)+(p-\widetilde{p})\right\| \\
& +2 \alpha_{n}\|(\gamma S-\mu F) p\|\left\|y_{n}-p\right\| .
\end{aligned}
$$


Since $\lim _{n \rightarrow \infty} \alpha_{n}=0$ and $\left\{x_{n}\right\},\left\{y_{n}\right\},\left\{w_{n}\right\}$, and $\left\{\tilde{v}_{n}\right\}$ are bounded sequences, by the assumption $\lim _{n \rightarrow \infty}\left\|x_{n}-y_{n}\right\|=0$, we conclude from (108) that

$$
\lim _{n \rightarrow \infty}\left\|\left(\widetilde{v}_{n}-w_{n}\right)+(p-\tilde{p})\right\|=0
$$

Note that

$$
\begin{aligned}
\left\|v_{n}-w_{n}\right\| \leq & \left\|\left(v_{n}-\widetilde{v}_{n}\right)-(p-\widetilde{p})\right\| \\
& +\left\|\left(\widetilde{v}_{n}-w_{n}\right)+(p-\widetilde{p})\right\| .
\end{aligned}
$$

Hence from (114) and (117) we get

$$
\lim _{n \rightarrow \infty}\left\|v_{n}-G v_{n}\right\|=\lim _{n \rightarrow \infty}\left\|v_{n}-w_{n}\right\|=0 .
$$

Also, observe that

$$
y_{n}=\alpha_{n} \gamma S v_{n}+\left(I-\alpha_{n} \mu F\right) W_{n} G v_{n} .
$$

Hence we get

$$
y_{n}-W_{n} G v_{n}=\alpha_{n}\left(\gamma S v_{n}-\mu F W_{n} G v_{n}\right) .
$$

So, from $\lim _{n \rightarrow \infty} \alpha_{n}=0$ and the boundedness of $\left\{v_{n}\right\}$ we deduce that

$$
\lim _{n \rightarrow \infty}\left\|y_{n}-W_{n} G v_{n}\right\|=0 .
$$

In addition, it is readily found that

$$
\begin{aligned}
&\left\|W_{n} v_{n}-v_{n}\right\| \\
& \leq\left\|W_{n} v_{n}-W_{n} G v_{n}\right\|+\left\|W_{n} G v_{n}-v_{n}\right\| \\
& \leq\left\|v_{n}-G v_{n}\right\|+\left\|W_{n} G v_{n}-v_{n}\right\| \\
& \leq\left\|v_{n}-G v_{n}\right\|+\left\|W_{n} G v_{n}-y_{n}\right\|+\left\|y_{n}-v_{n}\right\| \\
& \leq\left\|v_{n}-G v_{n}\right\|+\left\|W_{n} G v_{n}-y_{n}\right\| \\
&+\left\|y_{n}-x_{n}\right\|+\left\|x_{n}-v_{n}\right\| .
\end{aligned}
$$

Thus, by the assumption $\lim _{n \rightarrow \infty}\left\|x_{n}-y_{n}\right\|=0$, from (103) and (119)-(123) we have

$$
\lim _{n \rightarrow \infty}\left\|W_{n} v_{n}-v_{n}\right\|=0
$$

Taking into account that $\left\|v_{n}-W v_{n}\right\| \leq\left\|v_{n}-W_{n} v_{n}\right\|+\| W_{n} v_{n}-$ $W v_{n} \|$, we obtain from $\left\|v_{n}-W_{n} v_{n}\right\| \rightarrow 0$ and Remark 12 that

$$
\lim _{n \rightarrow \infty}\left\|v_{n}-W v_{n}\right\|=0 .
$$

Next, let us show that $\omega_{w}\left(x_{n}\right) \subset \Omega$. In fact, since $H$ is reflexive and $\left\{x_{n}\right\}$ is bounded, there exists at least a weak convergence subsequence of $\left\{x_{n}\right\}$. Hence it is known that $\omega_{w}\left(x_{n}\right) \neq \emptyset$. Now, take an arbitrary $w \in \omega_{w}\left(x_{n}\right)$. Then there exists a subsequence $\left\{x_{n_{i}}\right\}$ of $\left\{x_{n}\right\}$ such that $x_{n_{i}} \rightarrow w$. From (101) and (103) and the assumption $\lim _{n \rightarrow \infty}\left\|x_{n}-y_{n}\right\|=0$, we have that $y_{n_{i}} \rightarrow w$, $u_{n_{i}} \rightarrow w, v_{n_{i}} \rightarrow w, \Delta_{n_{i}}^{k} x_{n_{i}} \rightarrow w$, and $\Lambda_{n_{i}}^{m} u_{n_{i}} \rightarrow w$, where $k \in\{1,2, \ldots, M\}$ and $m \in\{1,2, \ldots, N\}$. Utilizing Lemma 9, we deduce from $x_{n_{i}} \rightarrow w, v_{n_{i}} \rightarrow w,(90)$, (119), and (125) that $w \in \operatorname{Fix}\left(P_{C}(I-(2 / L) \nabla f)\right)=\operatorname{VI}(C, \nabla f)=\Gamma, w \in \operatorname{GSVI}(G)$, and $w \in \operatorname{Fix}(W)=\cap_{n=1}^{\infty} \operatorname{Fix}\left(S_{n}\right)$ (due to Lemma 13). Thus, we get $w \in \cap_{n=1}^{\infty} \operatorname{Fix}\left(S_{n}\right) \cap \operatorname{GSVI}(G) \cap \Gamma$. Next we prove that $w \in \cap_{m=1}^{N} \operatorname{VI}\left(C, A_{m}\right)$. Let

$$
\widetilde{T}_{m} v= \begin{cases}A_{m} v+N_{C} v, & v \in C, \\ \emptyset, & v \notin C,\end{cases}
$$

where $m \in\{1,2, \ldots, N\}$. Let $(v, u) \in G\left(\widetilde{T}_{m}\right)$. Since $u-A_{m} v \in$ $N_{C} v$ and $\Lambda_{n}^{m} u_{n} \in C$, we have

$$
\left\langle v-\Lambda_{n}^{m} u_{n}, u-A_{m} v\right\rangle \geq 0 .
$$

On the other hand, from $\Lambda_{n}^{m} u_{n}=P_{C}\left(I-\lambda_{m, n} A_{m}\right) \Lambda_{n}^{m-1} u_{n}$ and $v \in C$, we have

$$
\left\langle v-\Lambda_{n}^{m} u_{n}, \Lambda_{n}^{m} u_{n}-\left(\Lambda_{n}^{m-1} u_{n}-\lambda_{m, n} A_{m} \Lambda_{n}^{m-1} u_{n}\right)\right\rangle \geq 0,
$$

and hence

$$
\left\langle v-\Lambda_{n}^{m} u_{n}, \frac{\Lambda_{n}^{m} u_{n}-\Lambda_{n}^{m-1} u_{n}}{\lambda_{m, n}}+A_{m} \Lambda_{n}^{m-1} u_{n}\right\rangle \geq 0 .
$$

Therefore we have

$$
\begin{aligned}
\langle v- & \left.\Lambda_{n_{i}}^{m} u_{n_{i}}, u\right\rangle \\
\geq & \left\langle v-\Lambda_{n_{i}}^{m} u_{n_{i}}, A_{m} v\right\rangle \\
\geq & \left\langle v-\Lambda_{n_{i}}^{m} u_{n_{i}}, A_{m} v\right\rangle \\
& -\left\langle v-\Lambda_{n_{i}}^{m} u_{n_{i}}, \frac{\Lambda_{n_{i}}^{m} u_{n_{i}}-\Lambda_{n_{i}}^{m-1} u_{n_{i}}}{\lambda_{m, n_{i}}}+A_{m} \Lambda_{n_{i}}^{m-1} u_{n_{i}}\right\rangle \\
= & \left\langle v-\Lambda_{n_{i}}^{m} u_{n_{i}}, A_{m} v-A_{m} \Lambda_{n_{i}}^{m} u_{n_{i}}\right\rangle \\
& +\left\langle v-\Lambda_{n_{i}}^{m} u_{n_{i}}, A_{m} \Lambda_{n_{i}}^{m} u_{n_{i}}-A_{m} \Lambda_{n_{i}}^{m-1} u_{n_{i}}\right\rangle \\
& -\left\langle v-\Lambda_{n_{i}}^{m} u_{n_{i}}, \frac{\Lambda_{n_{i}}^{m} u_{n_{i}}-\Lambda_{n_{i}}^{m-1} u_{n_{i}}}{\lambda_{m, n_{i}}}\right\rangle \\
\geq & \left\langle v-\Lambda_{n_{i}}^{m} u_{n_{i}}, A_{m} \Lambda_{n_{i}}^{m} u_{n_{i}}-A_{m} \Lambda_{n_{i}}^{m-1} u_{n_{i}}\right\rangle \\
& -\left\langle v-\Lambda_{n_{i}}^{m} u_{n_{i}}, \frac{\Lambda_{n_{i}}^{m} u_{n_{i}}-\Lambda_{n_{i}}^{m-1} u_{n_{i}}}{\lambda_{m, n_{i}}}\right\rangle .
\end{aligned}
$$

From (101) and since $A_{m}$ is Lipschitz continuous, we obtain that $\lim _{n \rightarrow \infty}\left\|A_{m} \Lambda_{n}^{m} u_{n}-A_{m} \Lambda_{n}^{m-1} u_{n}\right\|=0$. From $\Lambda_{n_{i}}^{m} u_{n_{i}} \rightarrow w$, $\left\{\lambda_{i, n}\right\} \subset\left[a_{i}, b_{i}\right] \subset\left(0,2 \eta_{i}\right)$, for all $i \in\{1,2, \ldots, N\}$ and (101), we have

$$
\langle v-w, u\rangle \geq 0
$$

Since $\widetilde{T}_{m}$ is maximal monotone, we have $w \in \widetilde{T}_{m}^{-1} 0$ and hence $w \in \mathrm{VI}\left(C, A_{m}\right), m=1,2, \ldots, N$, which implies $w \in \cap_{m=1}^{N} \operatorname{VI}\left(C, A_{m}\right)$. Next we prove that 
$w \in \cap_{k=1}^{M} \operatorname{GMEP}\left(\Theta_{k}, \varphi_{k}, B_{k}\right)$. Since $\Delta_{n}^{k} x_{n}=T_{r_{k, n}}^{\left(\Theta_{k}, \varphi_{k}\right)}(I-$ $\left.r_{k, n} B_{k}\right) \Delta_{n}^{k-1} x_{n}, n \geq 1, k \in\{1,2, \ldots, M\}$, we have

$$
\begin{aligned}
\Theta_{k}\left(\Delta_{n}^{k} x_{n}, y\right)+\varphi_{k}(y)-\varphi_{k}\left(\Delta_{n}^{k} x_{n}\right) & \\
+ & \left\langle B_{k} \Delta_{n}^{k-1} x_{n}, y-\Delta_{n}^{k} x_{n}\right\rangle \\
& +\frac{1}{r_{k, n}}\left\langle y-\Delta_{n}^{k} x_{n}, \Delta_{n}^{k} x_{n}-\Delta_{n}^{k-1} x_{n}\right\rangle \geq 0 .
\end{aligned}
$$

By (A2), we have

$$
\begin{aligned}
\varphi_{k}(y) & -\varphi_{k}\left(\Delta_{n}^{k} x_{n}\right)+\left\langle B_{k} \Delta_{n}^{k-1} x_{n}, y-\Delta_{n}^{k} x_{n}\right\rangle \\
& +\frac{1}{r_{k, n}}\left\langle y-\Delta_{n}^{k} x_{n}, \Delta_{n}^{k} x_{n}-\Delta_{n}^{k-1} x_{n}\right\rangle \\
\geq & \Theta_{k}\left(y, \Delta_{n}^{k} x_{n}\right) .
\end{aligned}
$$

Let $z_{t}=t y+(1-t) w$ for all $t \in(0,1]$ and $y \in C$. This implies that $z_{t} \in C$. Then, we have

$$
\begin{aligned}
\left\langle z_{t}-\right. & \left.\Delta_{n}^{k} x_{n}, B_{k} z_{t}\right\rangle \\
\geq & \varphi_{k}\left(\Delta_{n}^{k} x_{n}\right)-\varphi_{k}\left(z_{t}\right)+\left\langle z_{t}-\Delta_{n}^{k} x_{n}, B_{k} z_{t}\right\rangle \\
& -\left\langle z_{t}-\Delta_{n}^{k} x_{n}, B_{k} \Delta_{n}^{k-1} x_{n}\right\rangle \\
& -\left\langle z_{t}-\Delta_{n}^{k} x_{n}, \frac{\Delta_{n}^{k} x_{n}-\Delta_{n}^{k-1} x_{n}}{r_{k, n}}\right\rangle+\Theta_{k}\left(z_{t}, \Delta_{n}^{k} x_{n}\right) \\
= & \varphi_{k}\left(\Delta_{n}^{k} x_{n}\right)-\varphi_{k}\left(z_{t}\right) \\
& +\left\langle z_{t}-\Delta_{n}^{k} x_{n}, B_{k} z_{t}-B_{k} \Delta_{n}^{k} x_{n}\right\rangle \\
& +\left\langle z_{t}-\Delta_{n}^{k} x_{n}, B_{k} \Delta_{n}^{k} x_{n}-B_{k} \Delta_{n}^{k-1} x_{n}\right\rangle \\
& -\left\langle z_{t}-\Delta_{n}^{k} x_{n}, \frac{\Delta_{n}^{k} x_{n}-\Delta_{n}^{k-1} x_{n}}{r_{k, n}}\right\rangle+\Theta_{k}\left(z_{t}, \Delta_{n}^{k} x_{n}\right) .
\end{aligned}
$$

By (101), we have $\left\|B_{k} \Delta_{n}^{k} x_{n}-B_{k} \Delta_{n}^{k-1} x_{n}\right\| \rightarrow 0$ as $n \rightarrow \infty$. Furthermore, by the monotonicity of $B_{k}$, we obtain $\left\langle z_{t}-\right.$ $\left.\Delta_{n}^{k} x_{n}, B_{k} z_{t}-B_{k} \Delta_{n}^{k} x_{n}\right\rangle \geq 0$. Then, by (A4) we obtain

$$
\left\langle z_{t}-w, B_{k} z_{t}\right\rangle \geq \varphi_{k}(w)-\varphi_{k}\left(z_{t}\right)+\Theta_{k}\left(z_{t}, w\right) .
$$

Utilizing (A1), (A4), and (135), we obtain

$$
\begin{aligned}
0= & \Theta_{k}\left(z_{t}, z_{t}\right)+\varphi_{k}\left(z_{t}\right)-\varphi_{k}\left(z_{t}\right) \\
\leq & t \Theta_{k}\left(z_{t}, y\right)+(1-t) \Theta_{k}\left(z_{t}, w\right) \\
& +t \varphi_{k}(y)+(1-t) \varphi_{k}(w)-\varphi_{k}\left(z_{t}\right) \\
\leq & t\left[\Theta_{k}\left(z_{t}, y\right)+\varphi_{k}(y)-\varphi_{k}\left(z_{t}\right)\right] \\
& +(1-t)\left\langle z_{t}-w, B_{k} z_{t}\right\rangle \\
= & t\left[\Theta_{k}\left(z_{t}, y\right)+\varphi_{k}(y)-\varphi_{k}\left(z_{t}\right)\right] \\
& +(1-t) t\left\langle y-w, B_{k} z_{t}\right\rangle,
\end{aligned}
$$

and hence

$$
\begin{aligned}
0 \leq & \Theta_{k}\left(z_{t}, y\right)+\varphi_{k}(y)-\varphi_{k}\left(z_{t}\right) \\
& +(1-t)\left\langle y-w, B_{k} z_{t}\right\rangle .
\end{aligned}
$$

Letting $t \rightarrow 0$, we have, for each $y \in C$,

$$
0 \leq \Theta_{k}(w, y)+\varphi_{k}(y)-\varphi_{k}(w)+\left\langle y-w, B_{k} w\right\rangle .
$$

This implies that $w \in \operatorname{GMEP}\left(\Theta_{k}, \varphi_{k}, B_{k}\right)$ and hence $w \in$ $\cap_{k=1}^{M} \operatorname{GMEP}\left(\Theta_{k}, \varphi_{k}, B_{k}\right)$. Consequently, $w \in\left(\cap_{n=1}^{\infty} \operatorname{Fix}\left(S_{n}\right)\right) \cap$ $\left(\cap_{k=1}^{M} \operatorname{GMEP}\left(\Theta_{k}, \varphi_{k}, B_{k}\right)\right) \cap\left(\cap_{i=1}^{N} \operatorname{VI}\left(C, A_{i}\right)\right) \cap \operatorname{GSVI}(G) \cap \Gamma=:$ $\Omega$. (This shows that $\omega_{w}\left(x_{n}\right) \subset \Omega$.)

Step 4. We prove that $\omega_{w}\left(x_{n}\right) \subset \Xi$ provided that $\left\|x_{n}-y_{n}\right\|=$ $o\left(\alpha_{n}\right)$ additionally.

Indeed, let $w \in \omega_{w}\left(x_{n}\right)$ be the same as mentioned in Step 3 . Then we get $x_{n_{i}} \rightarrow w$. In addition, from (84) we have that for every $p \in \Omega$

$$
\begin{aligned}
\left\|y_{n}-p\right\|^{2} \leq & \left\|x_{n}-p\right\|^{2} \\
& +2 \alpha_{n}\left\langle(\gamma S-\mu F) p, y_{n}-p\right\rangle,
\end{aligned}
$$

which immediately implies that

$$
\begin{aligned}
2\langle(\gamma S & \left.-\mu F) p, p-y_{n}\right\rangle \\
& \leq \frac{1}{\alpha_{n}}\left(\left\|x_{n}-p\right\|^{2}-\left\|y_{n}-p\right\|^{2}\right) \\
& \leq \frac{\left\|x_{n}-y_{n}\right\|}{\alpha_{n}}\left(\left\|x_{n}-p\right\|+\left\|y_{n}-p\right\|\right) .
\end{aligned}
$$

This together with $\left\|x_{n}-y_{n}\right\|=o\left(\alpha_{n}\right)$ leads to

$$
\limsup _{n \rightarrow \infty}\left\langle(\gamma S-\mu F) p, p-y_{n}\right\rangle \leq 0 \text {. }
$$

Observe that

$$
\begin{aligned}
\limsup _{n \rightarrow \infty}\left\langle(\gamma S-\mu F) p, p-x_{n}\right\rangle \\
=\limsup _{n \rightarrow \infty}\left(\left\langle(\gamma S-\mu F) p, y_{n}-x_{n}\right\rangle\right. \\
\left.+\left\langle(\gamma S-\mu F) p, p-y_{n}\right\rangle\right) \\
=\limsup _{n \rightarrow \infty}\left\langle(\gamma S-\mu F) p, p-y_{n}\right\rangle \leq 0 .
\end{aligned}
$$

So, it follows from $x_{n_{i}} \rightarrow w$ that

$$
\langle(\gamma S-\mu F) p, p-w\rangle \leq 0, \quad \forall p \in \Omega .
$$

Also, note that $0<\gamma \leq \tau$ and

$$
\begin{aligned}
\mu \eta \geq \tau & \Longleftrightarrow \mu \eta \geq 1-\sqrt{1-\mu\left(2 \eta-\mu \kappa^{2}\right)} \\
& \Longleftrightarrow \sqrt{1-\mu\left(2 \eta-\mu \kappa^{2}\right)} \geq 1-\mu \eta \\
& \Longleftrightarrow 1-2 \mu \eta+\mu^{2} \kappa^{2} \geq 1-2 \mu \eta+\mu^{2} \eta^{2} \\
& \Longleftrightarrow \kappa^{2} \geq \eta^{2} \\
& \Longleftrightarrow \kappa \geq \eta .
\end{aligned}
$$


It is clear that

$$
\begin{aligned}
& \langle(\mu F-\gamma S) x-(\mu F-\gamma S) y, x-y\rangle \\
& \quad \geq(\mu \eta-\gamma)\|x-y\|^{2}, \quad \forall x, y \in H .
\end{aligned}
$$

Hence, it follows from $0<\gamma \leq \tau \leq \mu \eta$ that $\mu F-\gamma S$ is monotone. Since $w \in \omega_{w}\left(x_{n}\right) \subset \Omega$, by Minty's lemma [39] we have

$$
\langle(\gamma S-\mu F) w, p-w\rangle \leq 0, \quad \forall p \in \Omega ;
$$

that is, $w \in \Xi$. Therefore, $\omega_{w}\left(x_{n}\right) \subset \Xi$. This completes the proof.

Theorem 20. Assume that there hold all the conditions in Theorem 19. Then we have the following.

(i) $\left\{x_{n}\right\}$ converges strongly to a point $x^{*} \in \Omega$ provided that $\lim _{n \rightarrow \infty}\left\|x_{n}-y_{n}\right\|=0$, which is a unique solution of the VIP: $\left\langle(\gamma V-\mu F) x^{*}, p-x^{*}\right\rangle \leq 0$, for all $p \in \Omega$; equivalently,

$$
P_{\Omega}(I-(\mu F-\gamma V)) x^{*}=x^{*}
$$

(ii) $\left\{x_{n}\right\}$ converges strongly to a unique solution of THVI (24) provided that $\left\|x_{n}-y_{n}\right\|=o\left(\alpha_{n}\right)$ additionally.

Proof. Observe that

$$
\begin{aligned}
& \langle(\mu F-\gamma V) x-(\mu F-\gamma V) y, x-y\rangle \\
& \geq(\mu \eta-\gamma l)\|x-y\|^{2}, \quad \forall x, y \in H .
\end{aligned}
$$

Hence we know that $\mu F-\gamma V$ is $(\mu \eta-\gamma l)$-strongly monotone with constant $(\mu \eta-\gamma l)>0$. In the meantime, it is easy to see that $\mu F-\gamma V$ is $\mu \kappa+\gamma l$-Lipschitzian with constant $\mu \kappa+\gamma l>0$. Thus, there exists a unique solution $x^{*} \in \Omega$ of the VIP

$$
\left\langle(\gamma V-\mu F) x^{*}, p-x^{*}\right\rangle \leq 0, \quad \forall p \in \Omega .
$$

Equivalently, $x^{*}=P_{\Omega}(I-(\mu F-\gamma V)) x^{*}$. Now, let us show that

$$
\limsup _{n \rightarrow \infty}\left\langle(\gamma V-\mu F) x^{*}, x_{n}-x^{*}\right\rangle \leq 0 .
$$

Since $\left\{x_{n}\right\}$ is bounded, we may assume, without loss of generality, that there exists a subsequence $\left\{x_{n_{i}}\right\}$ of $\left\{x_{n}\right\}$ such that $x_{n_{i}} \rightarrow w$ and

$$
\begin{aligned}
\limsup _{n \rightarrow \infty} & \left\langle(\gamma V-\mu F) x^{*}, x_{n}-x^{*}\right\rangle \\
= & \lim _{i \rightarrow \infty}\left\langle(\gamma V-\mu F) x^{*}, x_{n_{i}}-x^{*}\right\rangle \\
= & \left\langle(\gamma V-\mu F) x^{*}, w-x^{*}\right\rangle .
\end{aligned}
$$

In terms of Theorem 19(ii), we know that $w \in \omega_{w}\left(x_{n}\right) \subset \Omega$. So, from (149) it follows that

$$
\begin{aligned}
\limsup _{n \rightarrow \infty} & \left\langle(\gamma V-\mu F) x^{*}, x_{n}-x^{*}\right\rangle \\
= & \left\langle(\gamma V-\mu F) x^{*}, w-x^{*}\right\rangle \leq 0 .
\end{aligned}
$$

Next, let us show that $\lim _{n \rightarrow \infty}\left\|x_{n}-x^{*}\right\|=0$. In fact, put $p=x^{*}$ in (84). Then from (54) we get

$$
\begin{aligned}
& \left\|x_{n+1}-x^{*}\right\|^{2} \\
& =\| s_{n} \gamma\left(V x_{n}-V x^{*}\right)+\left(I-s_{n} \mu F\right) T_{n} y_{n} \\
& -\left(I-s_{n} \mu F\right) x^{*}+s_{n}(\gamma V-\mu F) x^{*} \|^{2} \\
& \left.\leq \| s_{n} \gamma\left(V x_{n}-V x^{*}\right)+\left(I-s_{n} \mu F\right) T_{n} y_{n}-\left(I-s_{n} \mu F\right) x^{*}\right) \|^{2} \\
& +2 s_{n}\left\langle(\gamma V-\mu F) x^{*}, x_{n+1}-x^{*}\right\rangle \\
& \leq\left[s_{n} \gamma\left\|V x_{n}-V x^{*}\right\|\right. \\
& \left.+\left\|\left(I-s_{n} \mu F\right) T_{n} y_{n}-\left(I-s_{n} \mu F\right) x^{*}\right\|\right]^{2} \\
& +2 s_{n}\left\langle(\gamma V-\mu F) x^{*}, x_{n+1}-x^{*}\right\rangle \\
& \leq\left[s_{n} \gamma l\left\|x_{n}-x^{*}\right\|+\left(1-s_{n} \tau\right)\left\|y_{n}-x^{*}\right\|\right]^{2} \\
& +2 s_{n}\left\langle(\gamma V-\mu F) x^{*}, x_{n+1}-x^{*}\right\rangle \\
& =\left[s_{n} \tau \frac{\gamma l}{\tau}\left\|x_{n}-x^{*}\right\|+\left(1-s_{n} \tau\right)\left\|y_{n}-x^{*}\right\|\right]^{2} \\
& +2 s_{n}\left\langle(\gamma V-\mu F) x^{*}, x_{n+1}-x^{*}\right\rangle \\
& \leq s_{n} \tau \frac{(\gamma l)^{2}}{\tau^{2}}\left\|x_{n}-x^{*}\right\|^{2}+\left(1-s_{n} \tau\right)\left\|y_{n}-x^{*}\right\|^{2} \\
& +2 s_{n}\left\langle(\gamma V-\mu F) x^{*}, x_{n+1}-x^{*}\right\rangle \\
& \leq s_{n} \frac{(\gamma l)^{2}}{\tau}\left\|x_{n}-x^{*}\right\|^{2}+\left(1-s_{n} \tau\right) \\
& \times\left[\left\|x_{n}-x^{*}\right\|^{2}+2 \alpha_{n}\left\langle(\gamma S-\mu F) x^{*}, y_{n}-x^{*}\right\rangle\right] \\
& +2 s_{n}\left\langle(\gamma V-\mu F) x^{*}, x_{n+1}-x^{*}\right\rangle \\
& =\left(1-s_{n} \frac{\tau^{2}-(\gamma l)^{2}}{\tau}\right)\left\|x_{n}-x^{*}\right\|^{2} \\
& +2\left(1-s_{n} \tau\right) \alpha_{n}\left\langle(\gamma S-\mu F) x^{*}, y_{n}-x^{*}\right\rangle \\
& +2 s_{n}\left\langle(\gamma V-\mu F) x^{*}, x_{n+1}-x^{*}\right\rangle \\
& =\left(1-s_{n} \frac{\tau^{2}-(\gamma l)^{2}}{\tau}\right)\left\|x_{n}-x^{*}\right\|^{2} \\
& +s_{n} \frac{\tau^{2}-(\gamma l)^{2}}{\tau} \cdot \frac{\tau}{\tau^{2}-(\gamma l)^{2}} \\
& \times\left[2\left(1-s_{n} \tau\right) \frac{\alpha_{n}}{s_{n}}\left\langle(\gamma S-\mu F) x^{*}, y_{n}-x^{*}\right\rangle\right. \\
& \left.+2\left\langle(\gamma V-\mu F) x^{*}, x_{n+1}-x^{*}\right\rangle\right] .
\end{aligned}
$$


Since $\sum_{n=1}^{\infty} s_{n}=\infty, \lim _{n \rightarrow \infty}\left(\alpha_{n} / s_{n}\right)=0$, and $\lim \sup _{n \rightarrow \infty}\left\langle(\gamma V-\mu F) x^{*}, x_{n+1}-x^{*}\right\rangle \leq 0$ (due to (152)), we deduce that $\sum_{n=1}^{\infty} s_{n}\left(\left(\tau^{2}-(\gamma l)^{2}\right) / \tau\right)=\infty$ and

$$
\begin{aligned}
& \limsup _{n \rightarrow \infty} \frac{\tau}{\tau^{2}-(\gamma l)^{2}} \\
& \times\left[2\left(1-s_{n} \tau\right) \frac{\alpha_{n}}{s_{n}}\left\langle(\gamma S-\mu F) x^{*}, y_{n}-x^{*}\right\rangle\right. \\
&\left.\quad+2\left\langle(\gamma V-\mu F) x^{*}, x_{n+1}-x^{*}\right\rangle\right] \leq 0 .
\end{aligned}
$$

Therefore, applying Lemma 16 to (153) we infer that $\lim _{n \rightarrow \infty}\left\|x_{n}-x^{*}\right\|=0$.

On the other hand, let us suppose that $\left\|x_{n}-y_{n}\right\|=o\left(\alpha_{n}\right)$. Then by Theorem 19(iii) we know that $\omega_{w}\left(x_{n}\right) \subset \Xi$. Since $\mu F-$ $\gamma V: H \rightarrow H$ is $(\mu \kappa+\gamma l)$-Lipschitzian and $(\mu \eta-\gamma l)$-strongly monotone, there exists a unique solution $x^{*} \in \Xi$ of the VIP

$$
\left\langle\gamma V x^{*}-\mu F x^{*}, x-x^{*}\right\rangle \leq 0, \quad \forall x \in \Xi .
$$

Since the sequence $\left\{x_{n}\right\}$ is bounded, there exists a subsequence $\left\{x_{n_{i}}\right\}$ of $\left\{x_{n}\right\}$ such that

$$
\begin{aligned}
\limsup _{n \rightarrow \infty} & \left\langle(\gamma V-\mu F) x^{*}, x_{n}-x^{*}\right\rangle \\
= & \lim _{i \rightarrow \infty}\left\langle(\gamma V-\mu F) x^{*}, x_{n_{i}}-x^{*}\right\rangle .
\end{aligned}
$$

Also, since $H$ is reflexive and $\left\{x_{n}\right\}$ is bounded, without loss of generality we may assume that $x_{n_{i}}-\bar{x} \in \Xi$ (due to Theorem 19(iii)). Taking into account that $x^{*}$ is the unique solution of the VIP (155), we deduce from (156) that

$$
\begin{aligned}
\limsup _{n \rightarrow \infty} & \left\langle(\gamma V-\mu F) x^{*}, x_{n+1}-x^{*}\right\rangle \\
\leq & \left\langle(\gamma V-\mu F) x^{*}, \bar{x}-x^{*}\right\rangle \leq 0 .
\end{aligned}
$$

Repeating the same argument as in (153) we immediately conclude that

$$
\begin{aligned}
\| x_{n+1}- & x^{*} \|^{2} \\
\leq & \left(1-s_{n} \frac{\tau^{2}-(\gamma l)^{2}}{\tau}\right)\left\|x_{n}-x^{*}\right\|^{2} \\
& +s_{n} \frac{\tau^{2}-(\gamma l)^{2}}{\tau} \cdot \frac{\tau}{\tau^{2}-(\gamma l)^{2}} \\
& \times\left[2\left(1-s_{n} \tau\right) \frac{\alpha_{n}}{s_{n}}\left\langle(\gamma S-\mu F) x^{*}, y_{n}-x^{*}\right\rangle\right. \\
& \left.+2\left\langle(\gamma V-\mu F) x^{*}, x_{n+1}-x^{*}\right\rangle\right] .
\end{aligned}
$$

Repeating the same arguments as above, we can readily see that $\lim _{n \rightarrow \infty}\left\|x_{n}-x^{*}\right\|=0$. This completes the proof.

Remark 21. It is obvious that our iterative algorithm (54) is very different from Ceng and Al-Homidan's iterative one in
[23, Theorem 21] and Yao et al.'s iterative one (21). Here, the two-step iterative scheme in [33, Theorem 3.2] and the threestep iterative scheme in [23, Theorem 21] are combined to develop our four-step iterative scheme (54) for the THVI (24). It is worth pointing out that under the lack of the assumptions similar to those in [33, Theorem 3.2], for example, $\left\{x_{n}\right\}$ is bounded, $\operatorname{Fix}(T) \cap \operatorname{int} C \neq \emptyset$ and $\|x-T x\| \geq k \operatorname{Dist}(x, \operatorname{Fix}(T))$, for all $x \in C$ for some $k>0$, the sequence $\left\{x_{n}\right\}$ generated by (54) converges strongly to a point $x^{*} \in\left(\cap_{n=1}^{\infty} \operatorname{Fix}\left(S_{n}\right)\right) \cap$ $\left(\cap_{k=1}^{M} \operatorname{GMEP}\left(\Theta_{k}, \varphi_{k}, B_{k}\right)\right) \cap\left(\cap_{i=1}^{N} \operatorname{VI}\left(C, A_{i}\right)\right) \cap \operatorname{GSVI}(G) \cap \Gamma=:$ $\Omega$, which is a unique solution of the VIP: $\left\langle\gamma V x^{*}-\mu F x^{*}, x-\right.$ $\left.x^{*}\right\rangle \leq 0$, for all $x \in \Omega$; equivalently, $P_{\Omega}(I-(\mu F-\gamma V)) x^{*}=x^{*}$ (see Theorem 20(i)).

Remark 22. Our Theorems 19 and 20 improve, extend, supplement, and develop Yao et al. [33, Theorems 3.1 and 3.2] and Ceng and Al-Homidan [23, Theorem 21] in the following aspects.

(a) Our THVI (24) with the unique solution $x^{*} \in \Xi$ satisfying

$$
\begin{aligned}
x^{*}= & P_{\left(\cap_{n=1}^{\infty} \operatorname{Fix}\left(S_{n}\right)\right) \cap\left(\cap_{k=1}^{M} \operatorname{GMEP}\left(\Theta_{k}, \varphi_{k}, B_{k}\right)\right) \cap\left(\cap_{i=1}^{N} \operatorname{VI}\left(C, A_{i}\right)\right) \cap \operatorname{GSVI}(G) \cap \Gamma} \\
& \times(I-(\mu F-\gamma S)) x^{*}
\end{aligned}
$$

is more general than the problem of finding a point $\tilde{x} \in C$ satisfying $\tilde{x}=P_{\mathrm{Fix}(T)} S \tilde{x}$ in [33] and than the problem of finding a point $x^{*} \epsilon$ $\left(\cap_{k=1}^{M} \operatorname{GMEP}\left(\Theta_{k}, \varphi_{k}, B_{k}\right)\right) \cap\left(\cap_{i=1}^{N} \operatorname{VI}\left(C, A_{i}\right)\right) \cap \Gamma$ in $[23$, Theorem 21].

(b) Our four-step iterative scheme (54) for THVI (24) is more flexible, more advantageous, and more subtle than Ceng and Al-Homidan's three-step iterative one in [23, Theorem 21] and than Yao et al.s two-step iterative one (21) because it can be used to solve several kinds of problems, for example, the THVI, the HFPP, and the problem of finding a common point of five sets: $\cap_{n=1}^{\infty} \operatorname{Fix}\left(S_{n}\right), \cap_{k=1}^{M} \operatorname{GMEP}\left(\Theta_{k}, \varphi_{k}, B_{k}\right)$, $\cap_{i=1}^{N} \operatorname{VI}\left(C, A_{i}\right), \operatorname{GSVI}(G)$, and $\Gamma$. In addition, it also drops the crucial requirements that $\operatorname{Fix}(T) \cap \operatorname{int} C \neq \emptyset$ and $\|x-T x\| \geq k \operatorname{Dist}(x, \operatorname{Fix}(T))$, for all $x \in C$ for some $k>0$ in [33, Theorem 3.2(v)].

(c) The argument techniques in our Theorems 19 and 20 are very different from the argument ones in [33, Theorems 3.1 and 3.2] and from the argument ones in [23, Theorem 21] because we use the $W$ mapping approach to find the fixed points of infinitely many nonexpansive mappings $\left\{S_{n}\right\}_{n=1}^{\infty}$ (see Lemmas 10 and 13), the properties of resolvent operators and maximal monotone mappings (see Proposition 6 and Lemma 18), the fixed point equation equivalent to the GSVI (11) (see Proposition CWY), and the contractive coefficient estimates for the contractions associating with nonexpansive mappings (see Lemma 15);

(d) Compared with the proof in [23, Theorem 21], our proof (see the arguments in Theorem 19) makes use of 
Minty's Lemma [39] to derive $\omega_{w}\left(x_{n}\right) \subset \Xi$ because our Theorem 19 involves a quite complex problem, that is, the THVI (24). The THVI (24) involves the HFPP for the nonexpansive mapping $S$ and infinitely many nonexpansive mappings $\left\{S_{n}\right\}_{n=1}^{\infty}$, but the problem in [23, Theorem 21] involves no HFPP for nonexpansive mappings.

\section{Conflict of Interests}

The authors declare that there is no conflict of interests regarding the publication of this paper.

\section{Acknowledgments}

This research was partially supported by the National Science Foundation of China (11071169), Innovation Program of Shanghai Municipal Education Commission (09ZZ133), and Ph.D. Program Foundation of Ministry of Education of China (20123127110002). This work was supported partly by the National Science Council of the Republic of China. This research was partially supported by a Grant from NSC.

\section{References}

[1] J. L. Lions, Quelques Methodes De Resolution Des Problemes Aux Limites Non Lineaires, Dunod, Paris, Farnce, 1969.

[2] R. Glowinski, Numerical Methods For Nonlinear Variational Problems, Springer, New York, NY, USA, 1984.

[3] W. Takahashi, Nonlinear Functional Analysis, Yokohama, Yokohama, Japan, 2000.

[4] J. T. Oden, Quantitative Methods on Nonlinear Mechanics, Prentice-Hall, Englewood Cliffs, NJ, USA, 1986.

[5] E. Zeidler, Nonlinear Functional Analysis and Its Applications, Springer, New York, NY, USA, 1985.

[6] G. M. Korpelevich, "The extragradient method for finding saddle points and other problems," Matecon, vol. 12, pp. 747756, 1976.

[7] L.-C. Zeng and J.-C. Yao, "Strong convergence theorem by an extragradient method for fixed point problems and variational inequality problems," Taiwanese Journal of Mathematics, vol. 10, no. 5, pp. 1293-1303, 2006.

[8] J.-W. Peng and J.-C. Yao, "A new hybrid-extragradient method for generalized mixed equilibrium problems, fixed point problems and variational inequality problems," Taiwanese Journal of Mathematics, vol. 12, no. 6, pp. 1401-1432, 2008.

[9] L. C. Ceng, C. Y. Wang, and J. C. Yao, "Strong convergence theorems by a relaxed extragradient method for a general system of variational inequalities," Mathematical Methods of Operations Research, vol. 73, no. 2, pp. 153-157, 2011.

[10] L.-C. Ceng, Q. H. Ansari, and S. Schaible, "Hybrid extragradient-like methods for generalized mixed equilibrium problems, systems of generalized equilibrium problems and optimization problems," Journal of Global Optimization, vol. 53, no. 1, pp. 69-96, 2012.

[11] Y. Yao, Y.-C. Liou, and S. M. Kang, "Approach to common elements of variational inequality problems and fixed point problems via a relaxed extragradient method," Computers and Mathematics with Applications, vol. 59, no. 11, pp. 3472-3480, 2010.
[12] L. C. Ceng, Q. H. Ansari, M. M. Wong, and J. C. Yao, "Mann type hybrid extragradient method for variational inequalities, variational inclusions and fixed point problems," Fixed Point Theory, vol. 13, no. 2, pp. 403-422, 2012.

[13] L.-C. Ceng, Q. H. Ansari, and J.-C. Yao, "An extragradient method for solving split feasibility and fixed point problems," Computers and Mathematics with Applications, vol. 64, no. 4, pp. 633-642, 2012.

[14] L.-C. Ceng, Q. H. Ansari, and J.-C. Yao, "Relaxed extragradient methods for finding minimum-norm solutions of the split feasibility problem," Nonlinear Analysis, Theory, Methods and Applications, vol. 75, no. 4, pp. 2116-2125, 2012.

[15] L.-C. Ceng, N. Hadjisavvas, and N.-C. Wong, "Strong convergence theorem by a hybrid extragradient-like approximation method for variational inequalities and fixed point problems," Journal of Global Optimization, vol. 46, no. 4, pp. 635-646, 2010.

[16] L.-C. Ceng and J.-C. Yao, "A relaxed extragradient-like method for a generalized mixed equilibrium problem, a general system of generalized equilibria and a fixed point problem," Nonlinear Analysis, Theory, Methods and Applications, vol. 72, no. 3-4, pp. 1922-1937, 2010.

[17] L.-C. Ceng, Q. H. Ansari, and J.-C. Yao, "Relaxed extragradient iterative methods for variational inequalities," Applied Mathematics and Computation, vol. 218, no. 3, pp. 1112-1123, 2011.

[18] S. Imnang and S. Suantai, "Strong convergence theorems for a general system of variational inequality problems, mixed equilibrium problems and fixed points problems with applications," Mathematical and Computer Modelling, vol. 52, no. 9-10, pp. 1682-1696, 2010.

[19] L.-C. Ceng and A. Petruşel, "Relaxed extragradient-like method for general system of generalized mixed equilibria and fixed point problem," Taiwanese Journal of Mathematics, vol. 16, no. 2, pp. 445-478, 2012.

[20] N. Nadezhkina and W. Takahashi, "Weak convergence theorem by an extragradient method for nonexpansive mappings and monotone mappings," Journal of Optimization Theory and Applications, vol. 128, no. 1, pp. 191-201, 2006.

[21] S.-M. Guu, L.-C. Ceng, and J.-C. Yao, "Finding common solutions of a variational inequality, a general system of variational inequalities, and a fixed-point problem via a hybrid extragradient method," Fixed Point Theory and Applications, vol. 2011, Article ID 626159, 19 pages, 2011.

[22] L. C. Ceng, H.-Y. Hu, and M. M. Wong, "Strong and weak convergence theorems for generalized mixed equilibrium problem with perturbation and fixed pointed problem of infinitely many nonexpansive mappings," Taiwanese Journal of Mathematics, vol. 15, no. 3, pp. 1341-1367, 2011.

[23] L. C. Ceng and S. Al-Homidan, "Algorithms of common solutions for generalized mixed equilibria, variational inclusions, and constrained convex minimization," Abstract and Applied Analysis, vol. 2014, Article ID 132053, 25 pages, 2014.

[24] S. Takahashi and W. Takahashi, "Strong convergence theorem for a generalized equilibrium problem and a nonexpansive mapping in a Hilbert space," Nonlinear Analysis, Theory, Methods and Applications, vol. 69, no. 3, pp. 1025-1033, 2008.

[25] L.-C. Ceng and J.-C. Yao, "A hybrid iterative scheme for mixed equilibrium problems and fixed point problems," Journal of Computational and Applied Mathematics, vol. 214, no. 1, pp. 186201, 2008.

[26] V. Colao, G. Marino, and H.-K. Xu, "An iterative method for finding common solutions of equilibrium and fixed point 
problems," Journal of Mathematical Analysis and Applications, vol. 344, no. 1, pp. 340-352, 2008.

[27] L. C. Ceng, A. Petruşel, and J. C. Yao, "Iterative approaches to solving equilibrium problems and fixed point problems of infinitely many nonexpansive mappings," Journal of Optimization Theory and Applications, vol. 143, no. 1, pp. 37-58, 2009.

[28] S.-M. Guu, L.-C. Ceng, and J.-C. Yao, "Finding common solutions of a variational inequality, a general system of variational inequalities, and a fixed-point problem via a hybrid extragradient method," Fixed Point Theory and Applications, vol. 2011, Article ID 626159, 25 pages, 2011.

[29] R. U. Verma, "On a new system of nonlinear variational inequalities and associated iterative algorithms," Mathematical Sciences Research Hot-Line, vol. 3, no. 8, pp. 65-68, 1999.

[30] L. C. Ceng, S. Al-Homidan, and Q. H. Ansari, "Iterative algorithms with regularization for hierarchical variational inequality problems and convex minimization problems," Fixed Point Theory and Applications, vol. 2013, p. 284, 2013.

[31] H.-K. Xu, "Averaged mappings and the gradient-projection algorithm," Journal of Optimization Theory and Applications, vol. 150, no. 2, pp. 360-378, 2011.

[32] L.-C. Ceng, Q. H. Ansari, and J.-C. Yao, "Some iterative methods for finding fixed points and for solving constrained convex minimization problems," Nonlinear Analysis, Theory, Methods and Applications, vol. 74, no. 16, pp. 5286-5302, 2011.

[33] Y. Yao, Y.-C. Liou, and G. Marino, “Two-step iterative algorithms for hierarchical fixed point problems and variational inequality problems," Journal of Applied Mathematics and Computing, vol. 31, no. 1-2, pp. 433-445, 2009.

[34] H. Iiduka, "Strong convergence for an iterative method for the triple-hierarchical constrained optimization problem," Nonlinear Analysis, Theory, Methods and Applications, vol. 71, no. 12, pp. e1292-e1297, 2009.

[35] H. Iiduka, "Iterative algorithm for solving triple-hierarchical constrained optimization problem," Journal of Optimization Theory and Applications, vol. 148, no. 3, pp. 580-592, 2011.

[36] L.-C. Ceng, Q. H. Ansari, and J.-C. Yao, "Iterative methods for triple hierarchical variational inequalities in hilbert spaces," Journal of Optimization Theory and Applications, vol. 151, no. 3, pp. 489-512, 2011.

[37] C. Byrne, "A unified treatment of some iterative algorithms in signal processing and image reconstruction," Inverse Problems, vol. 20, no. 1, pp. 103-120, 2004.

[38] P. L. Combettes, "Solving monotone inclusions via compositions of nonexpansive averaged operators," Optimization, vol. 53, no. 5-6, pp. 475-504, 2004.

[39] K. Goebel and W. A. Kirk, Topics on Metric Fixed-Point Theory, Cambridge University Press, Cambridge, England, 1990.

[40] J. G. O’Hara, P. Pillay, and H.-K. Xu, "Iterative approaches to convex feasibility problems in Banach spaces," Nonlinear Analysis, Theory, Methods and Applications, vol. 64, no. 9, pp. 2022-2042, 2006.

[41] Y. Yao, Y.-C. Liou, and J.-C. Yao, "Convergence theorem for equilibrium problems and fixed point problems of infinite family of nonexpansive mappings," Fixed Point Theory and Applications, vol. 2007, Article ID 64363, 12 pages, 2007.

[42] H. K. Xu and T. H. Kim, "Convergence of hybrid steepestdescent methods for variational inequalities," Journal of Optimization Theory and Applications, vol. 119, no. 1, pp. 185-201, 2003.
[43] R. T. Rockafellar, "Monotone operators and the proximal point algorithms," SIAM Journal on Control and Optimization, vol. 14, no. 5, pp. 877-898, 1976.

[44] J.-B. Baillon and G. Haddad, "Quelques propriétés des opérateurs angle-bornés et n-cycliquement monotones," Israel Journal of Mathematics, vol. 26, no. 2, pp. 137-150, 1977. 


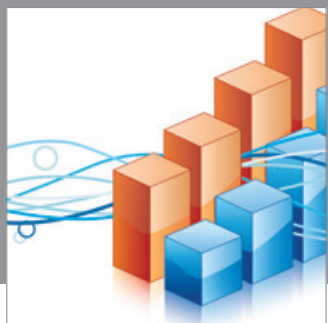

Advances in

Operations Research

mansans

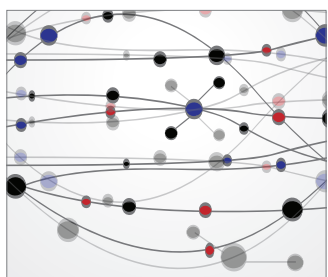

The Scientific World Journal
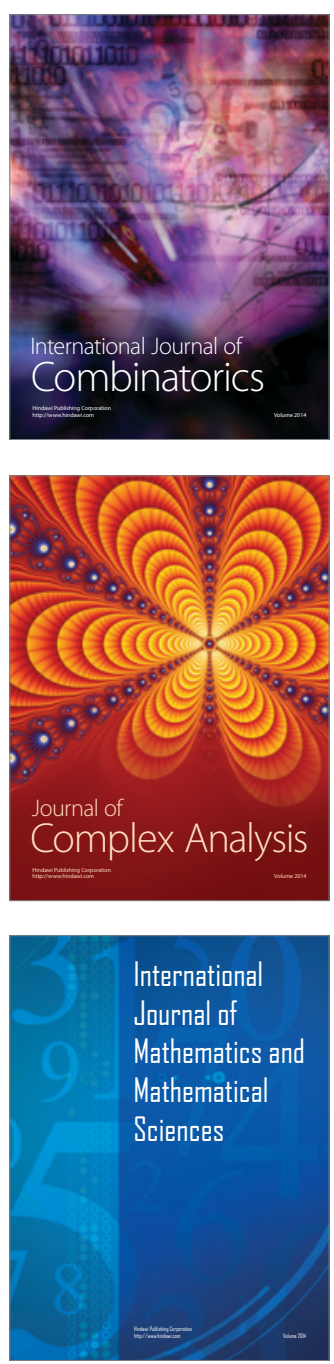
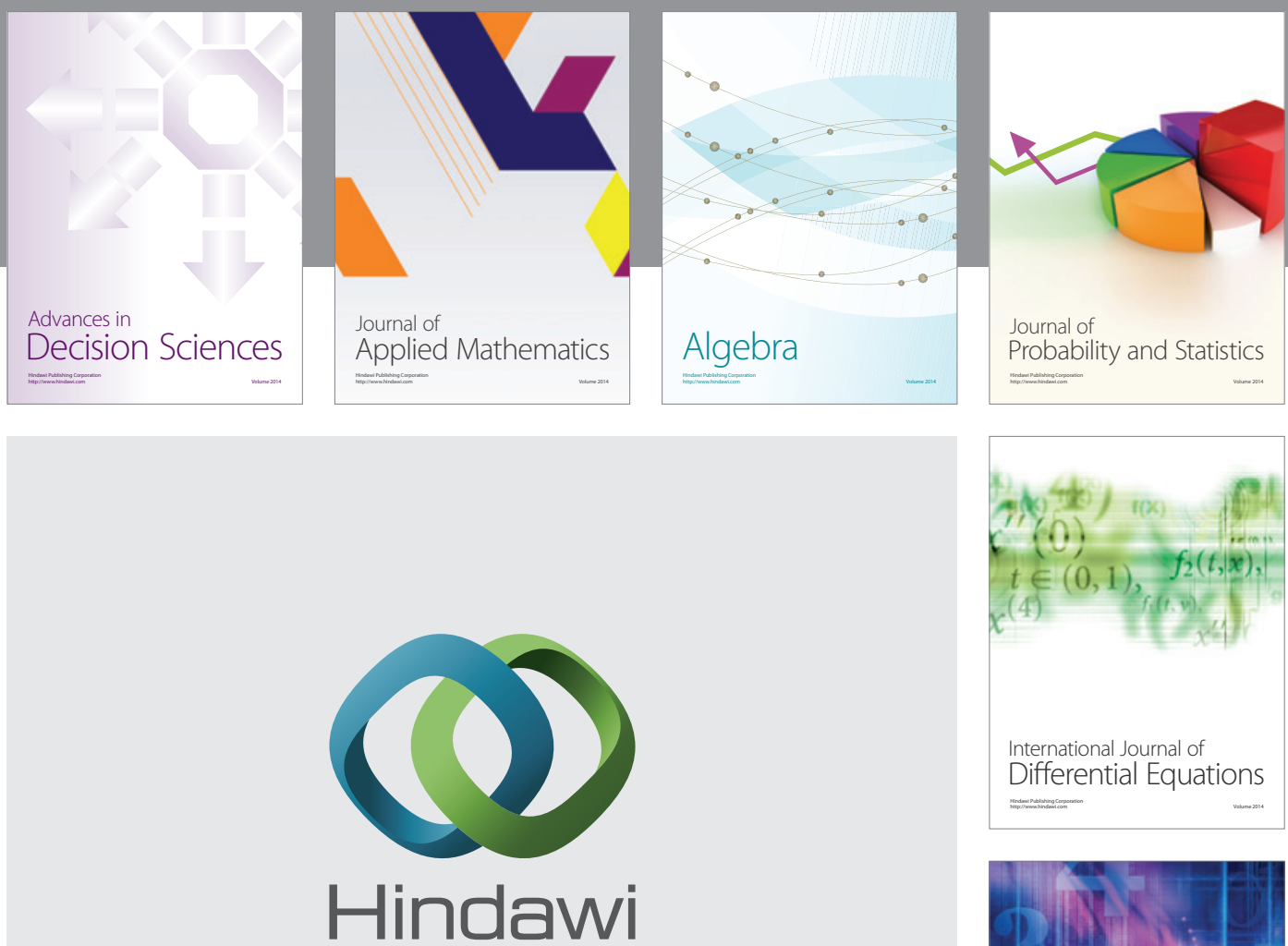

Submit your manuscripts at http://www.hindawi.com
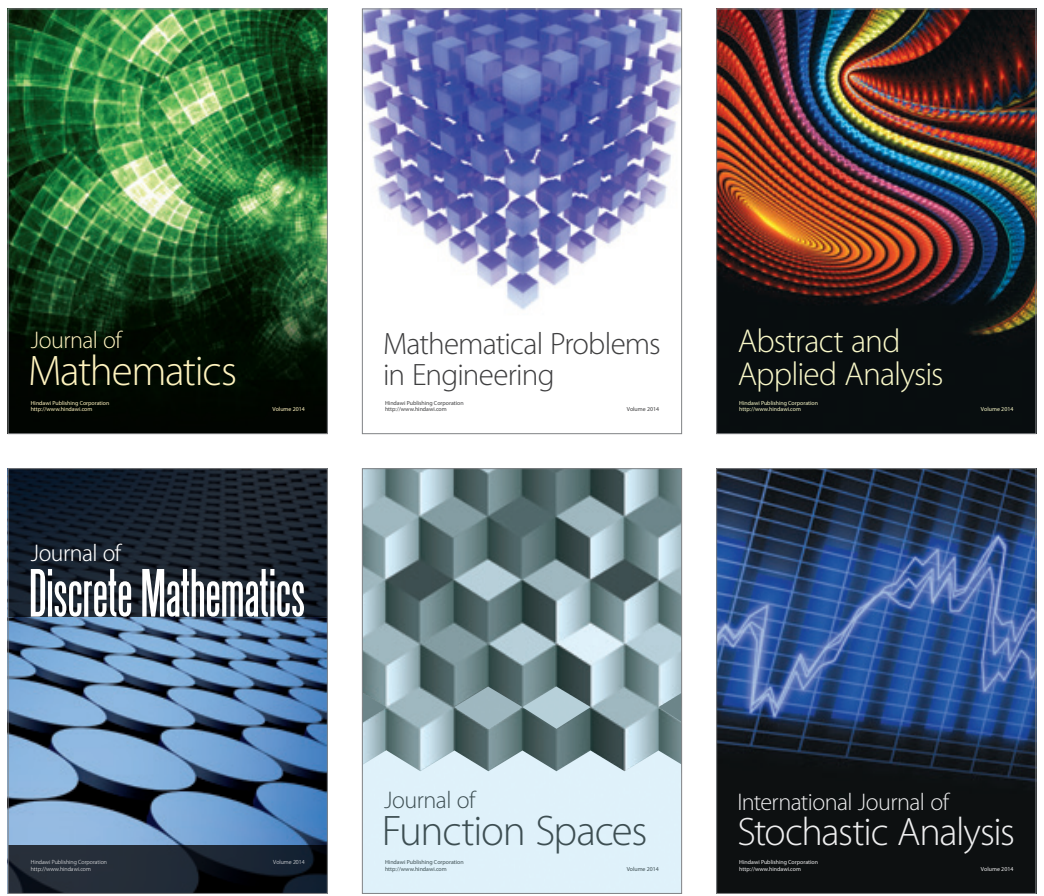

Journal of

Function Spaces

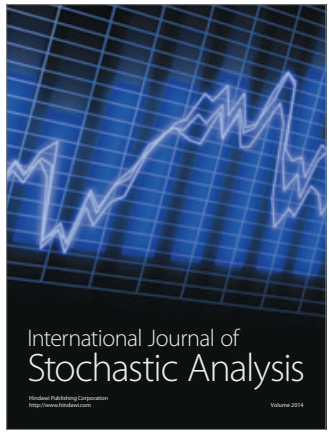

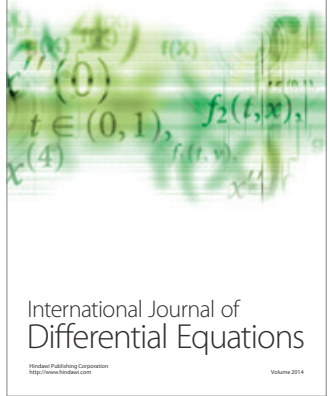
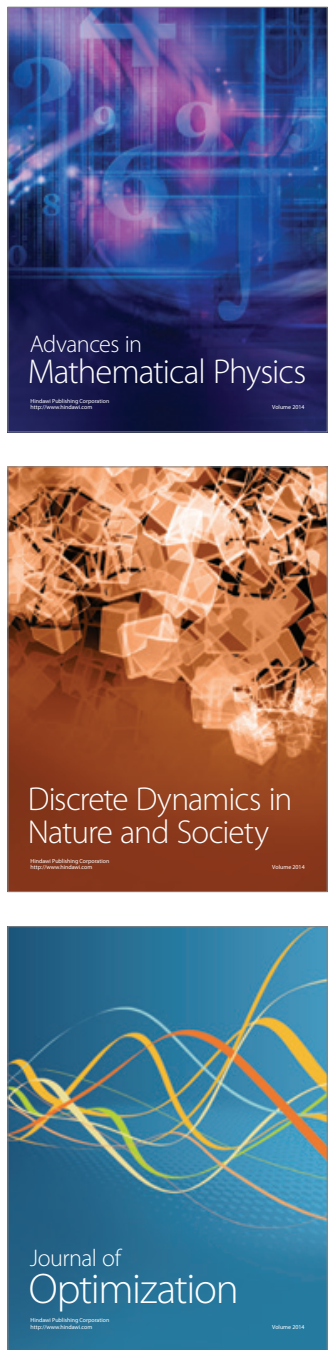